\begin{abstract}
EXPLOITATION OF NONLINEAR DYNAMICS OF BUCKLED BEAMS
\end{abstract}

\author{
by James Matthew Wilson
}

Axially-loaded structures play an integral role in engineering design. Some of these structures exhibit nonlinear response behavior under harmonic loading. Methods aimed at eliminating these behaviors are often employed in design. The question, however, arises: are there any beneficial behaviors that are unknowingly being eliminated? If so, can the nonlinear dynamics be exploited for design benefits? Our hypothesis is that the nonlinear dynamics can be used to optimize system response characteristics. In this thesis, the dynamic behavior of straight and buckled beams under harmonic excitation is considered. Beam models with various sources of nonlinearity are presented and numerical methods are employed to simulate system responses. An optimization approach is formulated that achieves maximized, periodic, and stable responses of the beam systems. Case studies are presented that demonstrate the ability, efficiency, and robustness of the optimization approach to exploit the nonlinear dynamics to achieve desired responses. 


\title{
EXPLOITATION OF NONLINEAR DYNAMICS OF BUCKLED BEAMS
}

\author{
A Thesis \\ Submitted to the \\ Faculty of Miami University \\ in partial fulfillment of \\ the requirements for the degree of \\ Master of Science \\ Department of Mechanical and Manufacturing Engineering \\ by \\ James Matthew Wilson \\ Miami University \\ Oxford, Ohio \\ 2015
}

Advisor

Dr. Amit Shukla

Reader

Dr. Timothy M. Cameron

Reader

Dr. Kumar V. Singh

Reader

Mr. William Olson 
(c)

James Matthew Wilson 2015 


\section{TABLE OF CONTENTS}

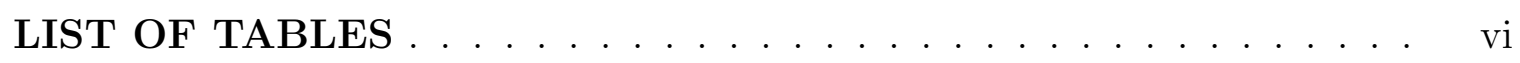

LIST OF FIGURES . . . . . . . . . . . . . . . vii

NOMENCLATURE ..................... ix

DEDICATION ...................... xi

ACKNOWLEDGEMENTS ................... xii

\section{CHAPTER}

I. Introduction $\ldots \ldots \ldots \ldots \ldots \ldots$

II. Literature Review . . . . . . . . . . . . . . . . . . . . 3

2.1 Straight Beams . . . . . . . . . . . . . . . . 3

2.2 Buckled Beams . . . . . . . . . . . . . . . . 5

2.3 Piezoelectric Hysteresis . . . . . . . . . . . . . . . . 6

2.4 Piezoelectric-Driven Beams . . . . . . . . . . . . . . 7

2.5 Optimization of Nonlinear Systems . . . . . . . . . 8

III. Beam Models . . . . . . . . . . . . . . . . . . . . . . . 11

3.1 Straight Beam Model: Small Deflections . . . . . . . . . . . 11

3.1.1 Multiple Mode Approximation . . . . . . . . . . . 14

3.2 Straight Beam Model: Large Deflections . . . . . . . . . . . 17

3.2.1 Multiple Mode Approximation . . . . . . . . . . . 20

3.3 Buckled Beam Model . . . . . . . . . . . . . . . . . 23

3.3.1 Buckled Beam Static Mode Shapes . . . . . . . . 25

3.3.2 Vibration About The Buckled Configuration . . . . 28

3.3.3 Multiple Mode Approximation . . . . . . . . . . . 29

3.4 Buckled Beam Model With Piezoelectric Excitation . . . . . . 30 
IV. Periodic Response . . . . . . . . . . . . . . . . . 35

4.1 Introduction . . . . . . . . . . . . . . . . 35

4.2 The Newton-Raphson Method . . . . . . . . . . . . . 37

4.3 The Shooting Method . . . . . . . . . . . . . . . . . 37

4.4 Sequential Continuation . . . . . . . . . . . . . . . . . 39

4.5 Arc-Length Continuation . . . . . . . . . . . . . . 41

4.5.1 Stability of Periodic Orbits . . . . . . . . . . 43

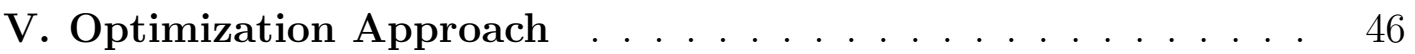

5.1 Motivation . . . . . . . . . . . . . . . . . 46

5.2 Problem Formulation . . . . . . . . . . . . . . 47

5.3 Optimization Algorithm Selection _. . . . . . . . . . 48

5.3.1 Scatter Search Optimization Algorithm (SSOA) . . 51

VI. Model Validation and Parametric Studies . . . . . . . . 54

6.1 Continuation Method Verification _... . . . . . . . 54

6.2 Nonlinear Straight Beam Model Response . . . . . . . . . . 56

6.2.1 Model Validation . . . . . . . . . . . . . . 58

6.2.2 Parametric Study: Beam Geometry . . . . . . . . 59

6.3 Buckled Beam . . . . . . . . . . . . . . . . . . . . 62

6.3.1 Model Validation ... . . . . . . . . . . . 64

6.3.2 Parametric Study: Buckling Level . . . . . . . . . 66

6.3.3 Three Mode Discretization . . . . . . . . . . . . . 68

VII. Optimization Case Studies _ . . . . . . . . . . . . 73

7.1 Optimization Method Verification _. . . . . . . . . 73

7.2 Optimization Case Studies: Harmonic Forcing . . . . . . . . 76

7.2.1 Case 1: Buckling Level and Beam Width . . . . . 76

7.2.2 Case 2: Beam Geometry, Buckling Level, Excitation Amplitude . . . . . . . . . . . . . . . 78

7.2.3 Case 3: Snap-Through Dynamics . . . . . . . . . 80

7.2.4 Case 4: Measurement Location and Buckling Level . 83

7.3 Optimization Case Studies: Hysteresis Forcing . . . . . . . 85

7.3.1 Piezoelectric Beam Nominal Response . . . . . . . . 86

7.3.2 Case 5: Piezoelectric Beam Geometry and Buckling Level . . . . . . . . . . . . . . . . . 89

7.3.3 Case 6: Piezoelectric Element Thickness . . . . . . . 91

7.3.4 Case 7: Piezoelectric Snap-Through Dynamics ... 93

7.4 Summary . . . . . . . . . . . . . . . . . . . 95 
VIII. Conclusions . . . . . . . . . . . . . . . . . . . . . . . . . 98

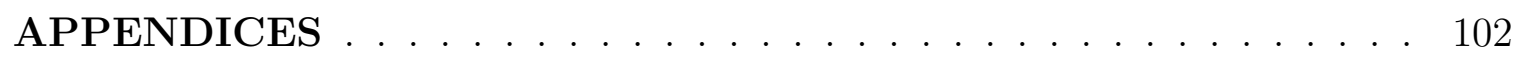

BIBLIOGRAPHY . . . . . . . . . . . . . . . . 105 


\section{LIST OF TABLES}

\section{Table}

$3.1 \quad$ Non-dimensional Natural Frequencies . . . . . . . . . . . . . . 15

3.2 Non-dimensional Critical Buckling Load Constants . . . . . . . . . . 28

$6.1 \quad$ Nominal Beam Dimensions and Material Properties . . . . . . . . 55

7.1 Solutions Found Via Optimization and Continuation Methods . . . 75

7.2 Case 1 Design Space . . . . . . . . . . . . . . . . 77

7.3 Solution Found Via Optimization . . . . . . . . . . . . 78

7.4 Case 2 Design Space . . . . . . . . . . . . . . 79

7.5 Solution Found Via Optimization . . . . . . . . . . . 81

7.6 Case 3 Design Space . . . . . . . . . . . . . . . . . . . . . 81

$7.7 \quad$ Solution Found Via Optimization . . . . . . . . . . . . . . 82

7.8 Case 4 Design Space . . . . . . . . . . . . . 83

7.9 Solution Found Via Optimization . . . . . . . . . . . 85

7.10 Piezoelectric Element Parameters . . . . . . . . . . . . . 86

7.11 Solution Found Via Optimization . . . . . . . . . . . . . . . 88

7.12 Case 5 Design Space . . . . . . . . . . . . . 89

7.13 Solution Found Via Optimization . . . . . . . . . . . . . 91

7.14 Case 6 Design Space . . . . . . . . . . . . . . 91

7.15 Solution Found Via Optimization . . . . . . . . . . . . . 93

7.16 Case 7 Design Space . . . . . . . . . . . . . . 94

7.17 Solution Found Via Optimization . . . . . . . . . . . . 95

7.18 Optimization Case Studies Summary _ . . . . . . . . . . . . 96

B.1 Buckled Beam Model Integration Constant Values . . . . . . . . . 104 


\section{LIST OF FIGURES}

\section{Figure}

$3.1 \quad$ Straight Beam Kinematic Diagram . . . . . . . . . . . . . . . . 12

3.2 Straight Beam (Small Deflection) Free Body Diagram . . . . . . . . 12

3.3 Straight Beam (Large Deflection) Free Body Diagram . . . . . . . . 17

$3.4 \quad$ Buckled Beam Kinematic Diagram . . . . . . . . . . . . . . . 23

3.5 Buckled Beam Free Body Diagram . . . . . . . . . . . . . . . 23

3.6 Piezoelectric Actuator Cutaway . . . . . . . . . . . . . . . . . . . 31

3.7 Various Hysteresis Models . . . . . . . . . . . . . . . . 32

$4.1 \quad$ Response After One Forcing Period . . . . . . . . . . . . . . . 38

4.2 Before Shooting Method . . . . . . . . . . . . . . 39

4.3 After Shooting Method . . . . . . . . . . . . . . . . 39

4.4 Sequential Continuation . . . . . . . . . . . . 40

4.5 Sequential Continuation for Strongly Nonlinear System . . . . . . . 41

4.6 Arc-Length Continuation for Nonlinear System . . . . . . . . . . . . 43

4.7 Stable Orbit . . . . . . . . . . . . . . . . 45

$4.8 \quad$ Unstable Orbit . . . . . . . . . . . . . . 45

$5.1 \quad$ Diverse Set of Trial Points . . . . . . . . . . . . . 52

$5.2 \quad$ Reference Set . . . . . . . . . . . . . 52

5.3 Local Gradient Optimization . . . . . . . . . . . . . . 53

5.4 Optimization Approach Overview . . . . . . . . . . . 53

$6.1 \quad$ Linear Frequency Response . . . . . . . . . . . . . 56

6.2 Large Deflection Beam Frequency Response (Single Mode Discretiza-

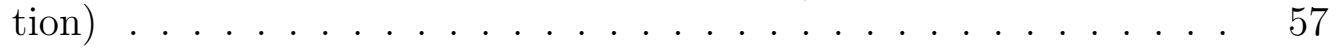

6.3 Large Deflection Beam Validation (Single Mode Discretization) . . . 59

6.4 Parametric Study: Effect of Beam Thickness . . . . . . . . . . 60

$6.5 \quad$ Beam Response Surface In $h-\Omega-A$ Space . . . . . . . . . . . . . . . 61 
6.6 Buckled Beam Frequency Response (Single Mode Discretization) . . 63

6.7 Buckled Beam Validation (Single Mode Discretization) . . . . . . . 64

6.8 Buckled Beam Model Validation (Two Mode Discretization) . . . . 65

6.9 Parametric Study: Effect of Static Rise . . . . . . . . . . . . . . 67

6.10 Buckled Beam Model (Three Mode Discretization) . . . . . . . . . . 69

6.11 First and Third Mode Shapes . . . . . . . . . . . . . 69

6.12 Buckled Beam (3 Mode Discretization) Two Parameter Study . . . 71

7.1 Optimization Method Verification (Measured At Beam Midpoint) . 75

7.2 Optimization Case 1 Result (Measured at Beam Midpoint) . . . . . 77

7.3 Optimization Case 2 Result (Measured at Beam Midpoint) . . . . . 80

7.4 Optimization Case 3 Result (Measured at Beam Midpoint) . . . . . 82

7.5 Optimization Case 4 Result (Measured at $\zeta=0.8$ ) . . . . . . . 84

$7.6 \quad$ First Mode Contribution . . . . . . . . . . . . . . . 86

7.7 Third Mode Contribution . . . . . . . . . . . . 86

7.8 Piezoelectric Buckled Beam Model . . . . . . . . . . . . . . . 87

$7.9 \quad$ Optimized Response . . . . . . . . . . . . . . . . . 88

7.10 Optimized Forcing Profile . . . . . . . . . . . . . 88

7.11 Optimization Nominal Case Result (Measured at Beam Midpoint) . 88

7.12 Optimized Response . . . . . . . . . . . . . . . . . 90

7.13 Optimized Forcing Profile . . . . . . . . . . . . . . 90

7.14 Optimization Case 5 Result (Measured at Beam Midpoint) . . . . . 90

7.15 Optimized Response . . . . . . . . . . . . . . 92

$7.16 \quad$ Optimized Forcing Profile . . . . . . . . . . . . . . . . . 92

7.17 Optimization Case 6 Result (Measured at Beam Midpoint) . . . . . 92

7.18 Optimized Response . . . . . . . . . . . . . . . . 94

7.19 Optimized Forcing Profile . . . . . . . . . . . . . 94

7.20 Optimization Case 7 Result (Measured at Beam Midpoint) . . . . . 94 


\section{NOMENCLATURE}

A :Beam cross-sectional area

$\alpha \quad$ :Hysteresis shape parameter

$b \quad$ :Buckled beam static midspan rise

$\beta \quad$ :Hysteresis shape parameter

$c \quad$ :Damping constant

$D \quad$ :Electric displacement

$d \quad$ :Piezoelectric constants

E :Beam elastic modulus

$E_{f} \quad$ :Electric field

$E_{P} \quad$ :Piezoelectric elastic modulus

$e^{T} \quad$ :Permittivity

$\epsilon_{m} \quad$ :Mechanical strain

$F \quad$ :Forcing amplitude

$F_{P}$ :Force due to single piezoelectric element

$f \quad$ :Transverse forcing function

$g \quad$ :Floquet stability function

$\gamma \quad$ :Hysteresis shape parameter

$H$ :Shooting function

$h$ :Beam thickness

I :Moment of inertia

$K^{*} \quad$ :Desired corrector steps

$K \quad$ :Required corrector steps

$k \quad:$ :Effective piezoelectric stiffness

$k_{n} \quad$ :Linear non-dimensional natural frequency

$L \quad$ :Beam length

$L_{P} \quad$ :Piezoelectric element length

$\lambda \quad$ :Non-dimensional critical buckling loads

$M \quad$ :Bending moment

$N \quad$ :Membrane force

$\Omega \quad$ :Forcing frequency

$\omega_{n} \quad$ :Natural frequency 


$\begin{array}{ll}P & : \text { External axial load } \\ P_{x_{0}} & : \text { Initial condition predictor vector } \\ P_{T} & : \text { Forcing frequency predictor vector } \\ P_{c r} & : \text { Non-dimensional critical buckling load } \\ \phi & : \text { Linear mode shape } \\ \Psi_{B} & : \text { Buckled static mode shape } \\ q_{n} & : \text { Generalized coordinate } \\ \boldsymbol{\rho} & : \text { Floquet multiplier vector } \\ \rho & : \text { Beam density } \\ s & : \text { Arc length continuation step size } \\ s^{E} & : \text { Mechanical compliance } \\ T & : \text { Time period } \\ u & : \text { Axial displacement of beam } \\ V & : \text { Shear force } \\ \boldsymbol{v} & : \text { Design variable vector } \\ v & : \text { Buckled beam response about buckled configuration } \\ w & : \text { Buckled beam total response } \\ w_{P} & : \text { Piezoelectric element width } \\ \bar{y} & : \text { Distance from piezoelectric neutral axis to beam neutral axis } \\ z & : \text { Hysteresis state variable } \\ \zeta & : \text { Non-dimensional beam length } \\ \zeta_{D} & : \text { Damping ratio } \\ & \end{array}$


To Daisy 


\section{ACKNOWLEDGEMENTS}

First and foremost, I would like to thank my advisor, Dr. Shukla for his expertise, patience, insight, and encouragement during the development of this thesis. I would like to my committee members, Dr. Cameron, Dr. Singh, and Mr. Olson for reading and evaluating my work as well as supporting my development as an engineer on a personal level. I thank the entire faculty in the Department of Mechanical and Manufacturing Engineering at Miami University for both my undergraduate and graduate level eduction. Additionally, I would like to express gratitude to my high school physics teacher, Dr. Oldaker, for showing me the beauty of classical mechanics and inspiring me to become an engineer.

I would also like to thank my parents, sister, and brother for their continued support. Finally, I would like to thank Mary for her steadfast encouragement, love,

and caring during my entire graduate program. My successful completion of the program and this thesis would not have been possible without her.

This work was partially supported by Ethicon Inc., a Johnson and Johnson company. 


\section{CHAPTER I}

\section{Introduction}

Many times, nonlinearities are seen as a burden in the design process as they can result in unpredictable and extremely sensitive responses. As such, engineers make design changes to effectively minimize or completely eliminate the nonlinear behavior. Are there, however, any beneficial characteristics in the nonlinearity that can be utilized or exploited for design? Our hypothesis is that the nonlinear dynamics of a piezoelectrically-excited, buckled beam, can be used to optimize system response characteristics.

Axial loaded beams are commonly used as components of mechanical systems and when dynamically excited, can produce complicated physics. Among these physics, nonlinear phenomena continue to be difficult to identify and quantify. For example, when straight beams are transversely deflected past a certain magnitude, linear beam models can produce incorrect results such as predicting deflection that exceeds the length of the beam-obviously an impossible feat. In order to better capture the physics of large beam deflection, one needs to consider both the transverse and axial motion of the beam. This consideration can lead to geometric nonlinearities and complex dynamic responses. In the case of a beam that has been axially loaded past a critical point, the beam will buckle. When the buckled beam is then transversely excited, both geometric and dynamic nonlinearities can arise. Furthermore, when 
excited by piezoelectric elements, hysteretic dynamic behavior of the piezoelectric material can add additional sources of nonlinearity to the complex beam system.

In this study, we begin by looking at past research on the relevant topics as discussed in Chapter II. We then derive four beam models: linear beam (small deflection), nonlinear beam (large deflection), buckled beam, and piezoelectrically-excited, buckled beam-all clamped at both ends. The derivations of these models are provided in Chapter III. The models produce sets of partial differential equations in both time and space that typically do not have exact or closed-form solutions. To mitigate this, we implement a method to transform these partial differential equations into sets of ordinary differential equations that can more easily be numerically integrated and simulated. In Chapter IV, a continuation technique is then introduced and implemented to produce nonlinear frequency response curves that provide insight to the dynamics of the systems. Chapter $\mathrm{V}$ addresses the issue of investigating the combined effects of parameter variations on the peak vibration amplitude when multiple system parameters are varied simultaneously. An optimization approach is formulated and presented that aims to obtain certain response characteristics as a function of system design variables. In Chapter VI, the beam models are validated and parametric studies are performed that highlight the effects of system parameters on the nonlinear beams' responses. Lastly, the optimization method is implemented in Chapter VII to optimize the nonlinear beam's responses as a function of several different design variables. The case studies serve as comprehensive demonstrations of exploiting the highly nonlinear, multi-physics dynamics of the beam models. 


\section{CHAPTER II}

\section{Literature Review}

In this chapter, the relevant past research is discussed. As a starting point, straight beam models (both linear and nonlinear) are first discussed as they lay the groundwork for more complicated beam configurations. Works that study the nonlinear dynamics of buckled beams are addressed next as vibrating buckled beams bring about additional nonlinearities in the system dynamics. The modeling of dynamic hysteresis behavior of piezoelectric elements is also discussed as much research as been performed to develop ways to accurately simulate piezoelectric nonlinearity. Past research into piezoelectric-beam systems is also explored as piezoelectric beams have been used extensively for both actuation and sensing. Lastly, works exploring optimization of nonlinear dynamic systems are highlighted.

\subsection{Straight Beams}

Present day beam theories use the same basic principles derived decades and centuries ago by Bernoulli, Euler, Coulomb, Poisson, Rayleigh, and Timoshenko, to name a few. In current literature and applications, the three most popular beam theories used are the Euler-Bernoulli beam theory, the Timoshenko beam theory, and the exact elasticity equations. Euler-Bernoulli beam theory was founded on two assumptions: the cross sections of the beam do not significantly deform under applied 
transverse or axial loads, and the material of the beam is linear elastic according to Hooke's law. The result of these assumptions is that Euler-Bernoulli beams do not experience transverse shear-deformation or transverse strains. These assumptions are valid for slender beams, and as such, Euler-Bernoulli beam theory is sometimes referred to as "Thin Beam Theory." In the case of thicker beams (beam thickness to length ratios greater than 0.01), transverse shear-deformation cannot be neglected. Incorporating the effects of transverse shear deformation into Euler-Bernoulli beam theory gives Timoshenko beam theory Timoshenko (1921). In Timoshenko beams, the shear strain is modeled as uniformly distributed over each beam cross section. Lastly, the least common beam theory, utilizing the exact elasticity equations for a given beam is rarely used as only a few problems can be solved exactly with this method Cowper (1968).

For thin beams with large deflections, the main assumptions of the Euler-Bernoulli beam theory do not suffice and consequently, nonlinear effects due to longitudinal stretching need to be accounted for. Tseng and Dugundji (1970) developed a nonlinear straight beam model and validated the simulated dynamic response experimentally. The main conclusion was that nonlinear straight beams experience nonlinear hardening under harmonic excitation. Similarly, Yamaki et al. (1980) developed a theoretical three degree of freedom nonlinear beam model that accounted for initial deflection and initial axial displacement. In agreement with Tseng and Dugundji (1970), a nonlinear hardening effect was seen. These findings were confirmed experimentally soon after the theoretical publication (Yamaki et al. (1980)). Both of these early models have served as starting points for more complicated models developed in recent literature. In a more recent study, Mestrom et al. (2008) developed a nonlinear microelectromechanical beam resonator using the Galerkin discretization method. Nonlinearities due to beam stretching as well as electromechanical coupling were incorporated into the model. Nonlinear hardening was observed and the model 
was validated against experimentation. A study by Barari et al. (2011) used perturbation methods to investigate the nonlinear vibrations of straight, large-deflection, Euler-Bernoulli beams. The perturbation method was compared against Galerkin discretization and found good agreement.

\subsection{Buckled Beams}

Tseng and Dugundji (1970) also modified the Euler-Bernoulli beam model to account for the nonlinear effects due to beam buckling. A nonlinear softening effect was seen in the dynamic response under harmonic excitation. The experimental findings were the first of their kind, exploring continuous dynamic snap-though. The nonlinear softening effect was attributed to a decrease of beam effective stiffness toward buckling. Abou-Rayan et al. (1993) studied a single-mode approximation of a parametrically excited hinged-hinged, buckled beam. The researchers found several nonlinear phenomena present in the beam's response including jump, period-multiplying bifurcations, period-demultiplying bifurcations, coexistence of periodic and chaotic attractors, chaotic snap-through, and local and global chaos. Nayfeh et al. (1995) developed exact solutions for linear beam vibrations about its $n$th buckling mode shape. The findings were experimentally validated. Lacarbonara (1997) presented a study on the stability and response of post-buckled beams. The findings highlighted the nonlinear softening effect of the model as well as modal cross-coupling giving rise to internal modal resonance. The work was validated experimentally. Investigating the response of buckled beams about higher buckling modes, Kreider and Nayfeh (1998) also experimentally validated calculated natural frequencies and mode shapes

of a clamped-clamped beam about the first buckling mode for various static buckling deflections. An increase in separation of the first and third vibration modes toward aggravated buckling was seen. A numerical study by Emam (2002) compared different solution techniques for simulating the response of a clamped clamped beam 
subject to transverse excitation. The findings showed that the Galerkin method converged to an acceptable solution in two to three retained modes. The study also developed expressions for the first four natural frequencies of a buckled beam as a function of the applied axial load. A relative separation of the first and third mode toward increased buckling was observed. Emam and Nayfeh (2004) also investigated the relationship between the applied buckling load and the beam's natural frequency. They found that with an increase in axial load past buckling gave rise to an increase in natural frequency of the asymmetric modes. He also found that at the point of buckling, the first natural frequency dropped to zero. As computational tools have evolved, model validation by means of finite element analysis has also been considered. Most recently, Formica et al. (2013) compared the nonlinear response of a transversely excited buckled beam found from model discretization to the response found via finite element analysis. The results showed excellent agreement between the two computational methods.

\subsection{Piezoelectric Hysteresis}

Piezoelectric materials have received much attention in the engineering community for their use as actuators and sensors. When subjected to relatively low input voltages (or input displacements), a linear piezoelectric response is typically observed. At higher excitation voltages (or displacements), however, a phase lagging response can be observed in the form of hysteresis (Ge and Jouaneh (1995)). While the origin of this nonlinear behavior is still the subject of many studies, the general consensus is that the nonlinear response is primarily due to internal material sliding within the piezoelectric crystal. This sliding action gives rise to residual misalignment of the piezoelectric crystal grains resulting in energy loss in the form of hysteresis $(G e$ and Jouaneh (1995), Ikeda (1996)). Regardless of the exact mechanism causing the nonlinearity, there has been extensive research exploring ways in which to model the 
hysteresis behavior of some piezoelectric materials. The most well known piezoelectric hysteresis model is the Preisach model (Preisach (1935)). The Preisach model has been implemented in numerous science and engineering applications. The model represents hysteresis as a continuous superposition of two-position relay operators. The Prandtl-Ishlinskii (PI) model improves on the Preisach model by superposing several Preisach "operator" to construct models of elasto-plasticity with nonlinearity due to strain-hardening (Kenmochi and Visintin (1994)). Several variations of the PI model have been implemented aiming to smooth out the rough hysteresis response that arises from using too few Preisach operators (Garmón et al. (2003), Bashash and Jalili (2007)). A different approach than the Preisach-based models is a model developed by Jouaneh and Tian (1992). In this model, a hysteresis state variable is introduced in the form of a first-order ordinary differential equation. Tuning parameters are included in the model to control the shape, amplitude, sharpness of backlash, as well as other shaping characteristics of the hysteresis. By using a least-squares or other curve fitting method, the tuning parameters can be adjusted to cause the hysteresis state variable to match with experimental data. One downside to using this model is that because the hysteresis is dependent on a state variable, the trajectory of the hysteresis is dictated by the initial conditions. For harmonic forcing models, this raises the issue of achieving a periodic hysteresis without any transient behavior.

\subsection{Piezoelectric-Driven Beams}

Beams and piezoelectric elements are often used together in the application of energy harvesting devices. DeVoe and Pisano (1997) modeled a piezoelectric cantilever beam and presented metrics for future optimization of the power output as a function of beam geometry. Elvin et al. (2001) modeled a cantilever beam with piezoelectric patches and attempted to optimize the wireless power transfer from

the device. Eggborn (2003) modeled a cantilever beam-piezo energy harvester and 
proposed optimization algorithms to optimize power harvesting. Similar studies for power optimization are numerous.

Li et al. (2006) developed mathematical models for piezoelectrically-actuated buckled beams and compared those with experimental data. Using the method of multiple scales, the researchers were able to study the effect of piezo-electrode thickness on the beam response. The findings showed high sensitivity to the piezo-electrode thickness that caused the system to bifurcate from a hardening response to a softening one. Waugh et al. (2011) exploited the nonlinear dynamics of a clamped clamped beam for viscosity and density sensing. The model measured the frequency separation between jump point in the frequency response function to measure various fluid flow characteristics. Applied to mass sensing, Kumar et al. (2011) developed both linear and nonlinear models of piezoelectrically-actuated microcantilevers. The authors proposed exploiting the nonlinear amplitude jump phenomena that occurs when mass is added to the system. This jump can then be used to detect the presence of a desired particulate. Nguyen et al. (2013), proposed the design of a bifurcation-based microelectromechanical mass detection device. The device exploited the nonlinear frequency shifting effect to detect mass on a clamped clamped microbeam. Addressing the material nonlinearities of piezoelectric ceramics, Dick (2013) characterized the bending moment provided by a PZT patch. The research found that this moment was a function of the axial loads on the patch as well as the patch geometry. This variable material property gave rise to system nonlinearity when used for actuation.

\subsection{Optimization of Nonlinear Systems}

Because of the design variable sensitivity typical of nonlinear dynamic systems, it can be difficult to achieve an optimal response. Kurdi et al. (2010) presented a novel approach to optimize the response amplitude of a nonlinear periodic system while ensuring minimum required input work. Motivated by finding efficient ways to drive 
micro air vehicles, the researchers formulated the optimization problem by defining forcing nodes (interpolated by cubic spline) and forcing frequency as design variables. The method required the specification of initial guesses for the design variables as well as the initial conditions. The Newton method was used to first optimize the initial conditions to obtain periodicity then a sequential quadratic programming scheme was used to adjust system parameters. After each adjustment of parameters, the Newton method was implemented to refine the solution to periodicity. The method was successful but did not consider the stability of the optimal solution. Brzeski et al. (2014) attempted to optimize the damping response of a Duffing-based tuned mass absorber. The model used the damping coefficient, the pendulum mass, as well as the forcing frequency as optimization design variables. The researchers used continuation methods to sweep through the entire design space recursively to develop response surfaces. They then implemented optimization methods to find the appropriate design variables that gave rise to the minimum response amplitude. This approach, while successful, was computationally expensive because of its need to explore the entire design space for an optimal solution. While successful for demonstrated examples, both the approaches used by Brzeski et al. (2014) and Kurdi et al. (2010) were computationally expensive as they relied on either full design space exploration or intermediate optimization to ensure periodicity.

A novel approach presented by Liao (2013) completely eliminated the need for both intermediate periodicity optimization as well as expensive total design space sweeps. The method aimed to maximize (or minimize) the response amplitude of a given nonlinear system as a function of the initial conditions and the excitation frequency, while ensuring periodicity and stability of the solution. This was accomplished by using a multi-start gradient descent algorithm and developing nonlinear equality and inequality constraints to ensure periodicity and stability. The method essentially was able to find various periodic responses along a frequency response 
curve without using any continuation technique. The work demonstrated the ability and efficiency of this method compared to continuation-based approaches. 


\section{CHAPTER III}

\section{Beam Models}

In this chapter, four beam models are presented. The first is a simple EulerBernoulli beam that undergoes small transverse deflection upon excitation. The second model is a modification of the Euler-Bernoulli model aiming to capture the internal axial stretching that occurs during large transverse deflection. The third beam model is that of a buckled beam which accounts for axial stretching as well as the nonlinear buckling phenomenon. The final beam model is an extension of the buckled beam model in which we model the hysteresis external excitation provided by piezoelectric patches. The modeling steps presented in this chapter follow the works of Tseng and Dugundji (1970), Emam (2002), Rao (2007), Virgin (2007), Nayfeh and Emam (2008), Waugh et al. (2011), and Younis (2011).

\subsection{Straight Beam Model: Small Deflections}

Consider a uniform, slender, clamped-clamped beam of length $L$, cross sectional area, $A$, excited with a distributed external force as shown in Fig. 3.1 where $w(x, t)$ represents the transverse displacement of the beam measured from the horizontal.

Inspecting a small rectangular portion of the beam, $d x$, gives the free body diagram as shown in Fig. 3.2 where $M(x, t)$ is the bending moment and $V(x, t)$ is the shear force. 


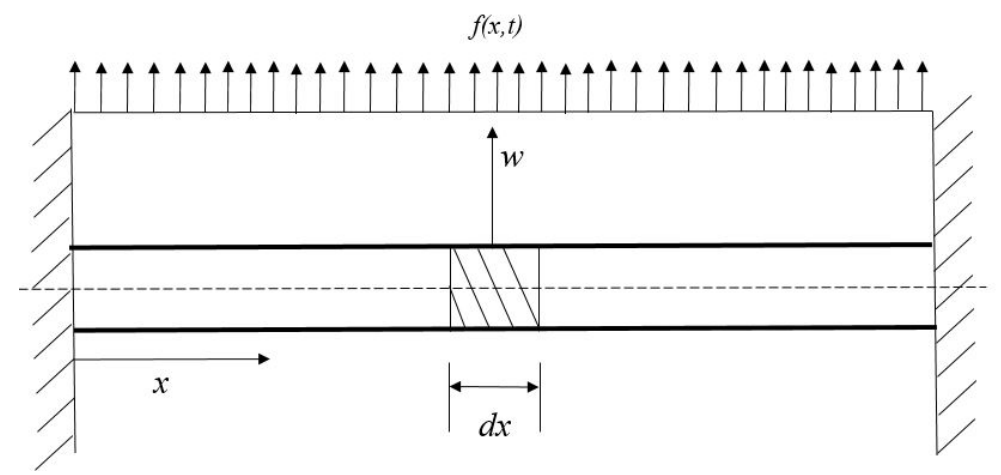

Figure 3.1: Straight Beam Kinematic Diagram

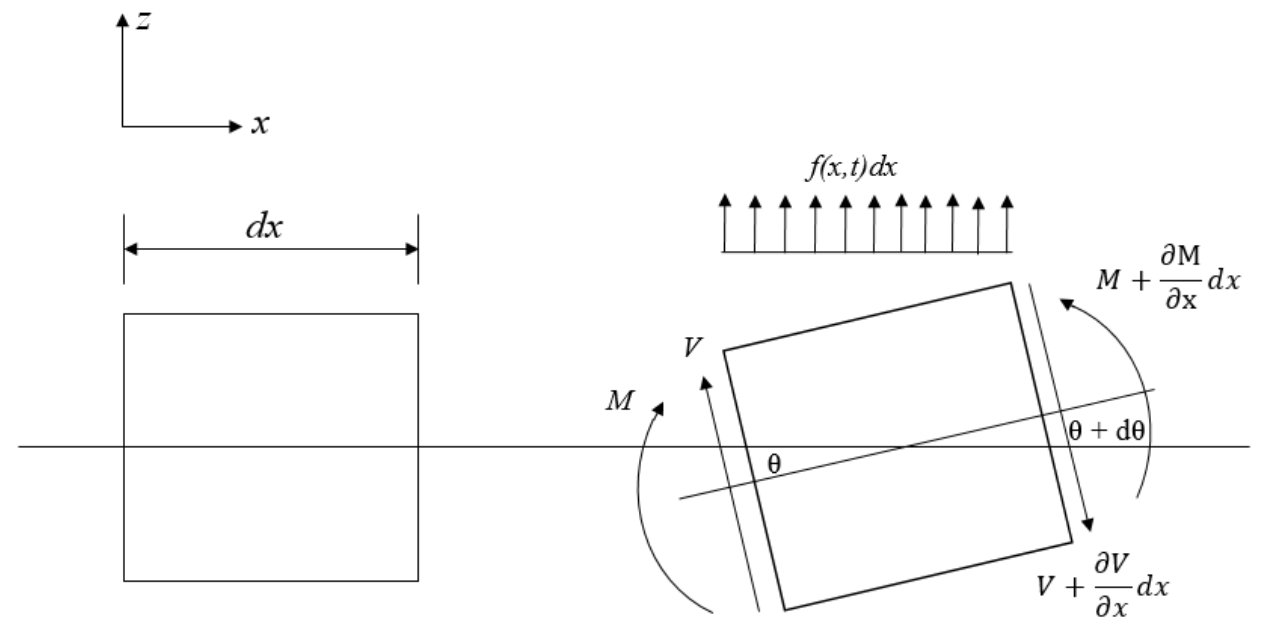

Figure 3.2: Straight Beam (Small Deflection) Free Body Diagram

Following Newton's Second Law of Motion, we balance the forces in the positive $z$ direction and equate them to an inertial term, giving

$$
-\left(V+\frac{\partial V}{\partial x} d x\right)+V+f(x, t)=\rho A d x \frac{\partial^{2} w}{\partial t^{2}}
$$

where $\rho$ is the mass density. Next, by summing the moments about the center of the element and ignoring rotary inertia (typically of negligible effect) gives

$$
\left(M+\frac{\partial M}{\partial x} d x\right)-M-\left(V+\frac{\partial V}{\partial x} d x\right) \frac{d x}{2}-V \frac{d x}{2}=0
$$

Simplifying Eq. 3.1 gives 


$$
-\frac{\partial V}{\partial x}+f(x, t)=\rho A \frac{\partial^{2} w}{\partial t^{2}}
$$

Similarly, simplifying Eq. 3.2 and ignoring terms of order $(d x)^{2}$ gives

$$
\frac{\partial M}{\partial x}-V(x, t)=0
$$

Next, by using the relation $V(x, t)=\frac{\partial M(x, t)}{\partial x}$, and substituting into Eq. 3.3 yields

$$
-\frac{\partial^{2} M}{\partial x^{2}}+f(x, t)=\rho A \frac{\partial^{2} w}{\partial t^{2}}
$$

From elementary theory of beam bending, the relationship between beam deflection and bending moment is given as

$$
M(x, t)=E I \frac{\partial^{2} w(x, t)}{\partial x^{2}}
$$

where $E$ is the Elastic modulus of the beam material and $I$ is the moment of inertia of the cross section of the beam about the y axis. Substituting Eq. 3.6 into Eq. 3.5 and rearranging gives the equation of motion for forced transverse vibration of a slender beam

$$
\frac{\partial^{2}}{\partial x^{2}}\left[E I \frac{\partial^{2} w(x, t)}{\partial x^{2}}\right]+\rho A \frac{\partial^{2} w}{\partial t^{2}}=f(x, t)
$$

For a beam clamped at each end, the boundary conditions are given as

$$
\begin{gathered}
w(0, t)=0 \\
\frac{\partial w(0, t)}{\partial x}=0 \\
w(L, t)=0
\end{gathered}
$$




$$
\frac{\partial w(L, t)}{\partial x}=0
$$

Eq. 3.7-3.11 give the basic Euler-Bernoulli beam model. While there are many ways to solve Eq. 3.7, we will use a reduced order technique via the Galerkin Discretization Method (Younis (2011)). The details of this method are presented next.

\subsubsection{Multiple Mode Approximation}

Analytical solutions exist for solving the equations of motion for the basic EulerBernoulli beam model. An alternate method can prove to be an effective solution technique when analytical solutions do not exist, as in more complicated models. This technique is called the Galerkin Discretization Method. In the Galerkin Discretization Method, the partial differential equations of a given system are solved by discretizing the system via superposition of a specified number of mode shapes. The result is a tunable approximation (tunable because the accuracy of the model is dictated by the number of mode shapes retained), that is represented by ordinary differential equations. These differential equations can then be numerically integrated to simulate

the system response. For ease of derivation, the following dimensionless variable is introduced: $\zeta=\frac{x}{L}$, yielding

$$
E I \frac{\partial^{4} w}{\partial \zeta^{4}}+\rho A \frac{\partial^{2} w}{\partial t^{2}}=f(\zeta, t)
$$

The Galerkin method assumes that the solution of 3.12 takes the form of

$$
w(\zeta, t)=\sum_{n=1}^{N} \phi_{n}(\zeta) q_{n}(t)
$$

where $\mathrm{N}$ is the number of modes included in the discretization, $\phi_{n}(x)$ are the linear vibrational mode shapes, and $q_{n}(t)$ are generalized coordinates. For example, $q_{1}(t)$ would represent the dynamics of the first mode in time domain. Multiplying this response by a scaling factor (specifically, the mode shape amplitude at $\zeta$ : $\phi_{1}(\zeta)$ ), 
gives the total dynamic response due to the first vibrational mode at a specific spacial location. The linear mode shapes are found from the eigenvalue problem presented by Eq. 3.12 and as such they are orthogonal:

$$
\int_{0}^{1} \phi_{i} \phi_{j} d \zeta=\delta_{i j}= \begin{cases}1, & \text { if } i=j \\ 0, & \text { if } i \neq j .\end{cases}
$$

Where $\delta_{i j}$ is the Kronecker delta function. For orthogonal mode shapes, the following relationship exists (Younis (2011))

$$
\int_{0}^{1} \phi_{j} \phi_{j}^{\prime \prime \prime \prime} d \zeta=k_{j}^{2}
$$

where $k_{j}$ is the $j^{t h}$ non-dimensional natural frequency. For straight, clamped beams, the linear, orthogonal mode shapes are given as (Rao (2007))

$$
\phi_{n}(\zeta)=\cosh \left(k_{n} \zeta\right)-\cos \left(k_{n} \zeta\right)-\alpha_{n}\left[\sinh \left(k_{n} \zeta\right)-\sin \left(k_{n} \zeta\right)\right]
$$

where

$$
\alpha=\frac{\sinh \left(k_{n}\right)+\sin \left(k_{n}\right)}{\cosh \left(k_{n}\right)-\cos \left(k_{n}\right)}
$$

and $n$ is the mode of interest. Values of $k$ for the first five modes of vibration are given in Table 3.1.1.

\begin{tabular}{|cc|}
\hline Mode & $k$ \\
\hline 1 & 4.73004 \\
2 & 7.85321 \\
3 & 10.9956 \\
4 & 14.1371 \\
5 & 17.2787 \\
\hline
\end{tabular}

Table 3.1: Non-dimensional Natural Frequencies

Returning to the original equation of motion, Eq. 3.13 is substituted into Eq. 


\subsection{2 giving}

$$
E I(\zeta) \sum_{n=1}^{N}\left[q_{n} \frac{d^{4} \phi_{n}}{d \zeta^{4}}\right]+\rho A \sum_{n=1}^{N}\left[\phi_{n} \frac{d^{2} q_{n}}{d t^{2}}\right]=f(\zeta, t)
$$

The Galerkin method is then implemented by multiplying Eq. 3.18 by $\phi_{m}$ and integrating from 0 to 1 with respect to $\zeta$. $\phi_{m}$ represents an arbitrary mode shape used to produce an equation for the $n$th mode where $m \neq n$. This process gives

$$
\int_{0}^{1}\left[\phi_{m}\left(E I \sum_{n=1}^{N}\left[q_{n} \frac{d^{4} \phi_{n}}{d \zeta^{4}}\right]+\rho A \sum_{n=1}^{N}\left[\phi_{n} \frac{d^{2} q_{n}}{d t^{2}}\right]=f(\zeta, t)\right)\right] d \zeta
$$

Distributing the integral and $\phi_{m}$ over each term gives

$$
E I \sum_{n=1}^{N} q_{n} \int_{0}^{1} \phi_{m} \frac{d^{4} \phi_{n}}{d \zeta^{4}} d \zeta+\rho A \sum_{n=1}^{N} \frac{d^{2} q_{n}}{d t^{2}} \int_{0}^{1} \phi_{m} \phi_{n} d \zeta=f(\zeta, t) \int_{0}^{1} \phi_{m} d \zeta
$$

Replacing spacial derivatives with "prime" superscripts and temporal derivatives with "dot" superscripts yields

$$
E I \sum_{n=1}^{N} q_{n} \int_{0}^{1} \phi_{m} \phi_{n}^{\prime \prime \prime \prime} d \zeta+\rho A \sum_{n=1}^{N} \ddot{q}_{n} \int_{0}^{1} \phi_{m} \phi_{n} d \zeta=f(\zeta, t) \int_{0}^{1} \phi_{m} d \zeta
$$

To make use of the orthogonality of the mode shapes and simplify, Eq. 3.21, we see that the orthogonality condition is only valid when $n=m$. Therefore, we make use of the orthogonality of the mode shapes (Eq. 3.14, Eq. 3.15), and adjust the indices on the summation terms:

$$
\rho A \ddot{q_{m}}+E I k_{m}^{2} q_{m}=A_{m} f(\zeta, t)
$$

where

$$
A_{m}=\int_{0}^{1} \phi_{m} d \zeta
$$


As evident in Eq. 3.22, the discretization process produces a system of ordinary differential equations that can be solved using various integration techniques. Furthermore, without any damping in the system, Eq. 3.22 represents the equations of a simple harmonic oscillator.

\subsection{Straight Beam Model: Large Deflections}

In the case of large transverse deflections, the Euler-Bernoulli assumption of no internal stretching does not suffice. To adjust for this physics, the free body diagram of Fig. 3.2 is adjusted to account for internal normal forces. Figure 3.3 shows this modification.

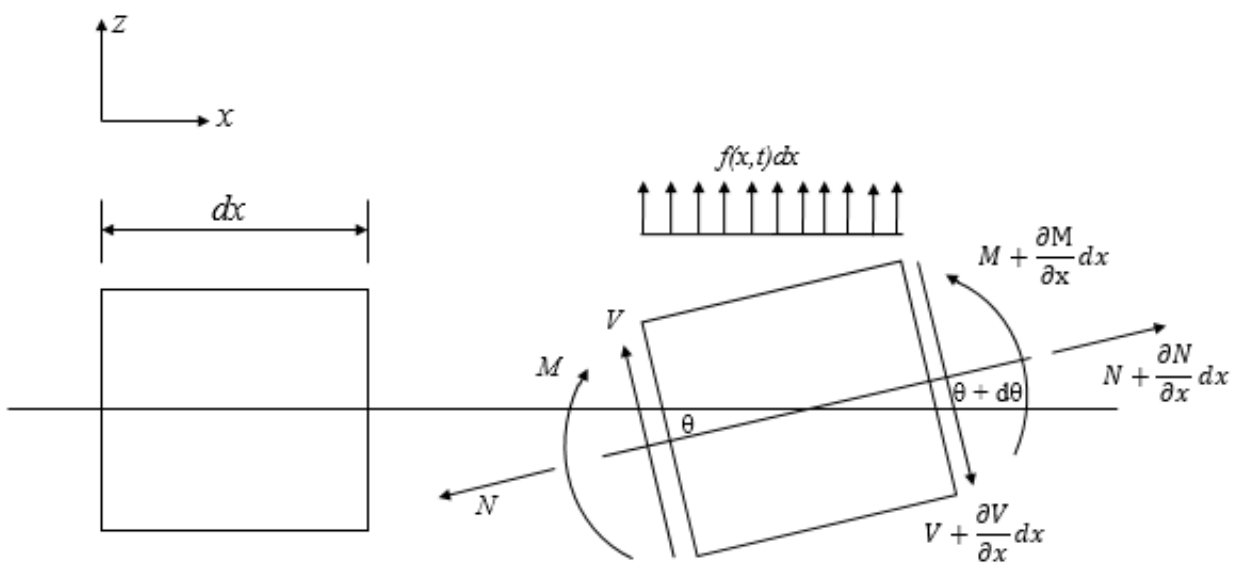

Figure 3.3: Straight Beam (Large Deflection) Free Body Diagram

The difference to note in Fig. 3.3 is the addition of the normal force, $N$, acting perpendicular to the beam element face. Deriving the equation of motion for a slender beam undergoing large transverse displacement is a similar process as that given in the previous section. Balancing the forces in the positive $z$ direction and equating to an inertial term gives 


$$
-\left(V+\frac{\partial V}{\partial x} d x\right)+V+\left(N+\frac{\partial N}{\partial x} d x\right) \sin (\theta+d \theta)-N \sin (\theta)+f(x, t)=\rho A \frac{\partial^{2} w}{\partial t^{2}}
$$

Simplifying gives

$$
-\frac{\partial V}{\partial x} d x+\left(N+\frac{\partial N}{\partial x} d x\right) \sin (\theta+d \theta)-N \sin (\theta)+f(x, t)=\rho A \frac{\partial^{2} w}{\partial t^{2}}
$$

Using the small angle approximation: $\sin (\theta)=\theta$ and $\sin (\theta+d \theta)=\theta+d \theta$ along with the substitution of $\theta=\frac{\partial w}{\partial x}$ and $d \theta=\frac{\partial^{2} w}{\partial x^{2}} d x$ gives

$$
-\frac{\partial V}{\partial x} d x+\left(N+\frac{\partial N}{\partial x} d x\right)\left(\frac{\partial w}{\partial x}+\frac{\partial^{2} w}{\partial x^{2}} d x\right)-N \frac{\partial w}{\partial x}+f(x, t)=\rho A \frac{\partial^{2} w}{\partial t^{2}}
$$

Simplifying Eq. 3.26 and ignoring terms of order $(d x)^{2}$ gives

$$
-\frac{\partial V}{\partial x}+N \frac{\partial^{2} w}{\partial x^{2}}+f(x, t)=\rho A \frac{\partial^{2} w}{\partial t^{2}}
$$

Summing the moments about the center of the element and ignoring rotary inertia (typically of negligible effect) gives

$$
\begin{gathered}
-\left(M+\frac{\partial M}{\partial x} d x\right)+M+\left(V+\frac{\partial V}{\partial x} d x\right) \frac{d x}{2}+V \frac{d x}{2}=0 \\
V(x, t)=\frac{\partial M}{\partial x}
\end{gathered}
$$

Inserting Eq. 3.29 into Eq. 3.27 and rearranging gives 


$$
-\frac{\partial^{2} M}{\partial x^{2}}+N \frac{\partial^{2} w}{\partial x^{2}}+f(x, t)=\rho A \frac{\partial^{2} w}{\partial t^{2}}
$$

From elementary theory of beam bending, the relationship between beam deflection and bending moment is given as

$$
M(x, t)=E I \frac{\partial^{2} w(x, t)}{\partial x^{2}}
$$

Substituting Eq. 3.31 into Eq. 3.30 and rearranging gives

$$
\frac{\partial^{2}}{\partial x^{2}}\left[E I \frac{\partial^{2} w}{\partial x^{2}}\right]+\rho A \frac{\partial^{2} w(x, t)}{\partial t^{2}}-N \frac{\partial^{2} w}{\partial x^{2}}=f(x, t)
$$

In order to account for any type of dissipative, non-restoring forces in the system, Eq. 3.32 can be modified to incorporate a velocity-dependent damping term

$$
\frac{\partial^{2}}{\partial x^{2}}\left[E I \frac{\partial^{2} w}{\partial x^{2}}\right]+\rho A \frac{\partial^{2} w}{\partial t^{2}}-N \frac{\partial^{2} w}{\partial x^{2}}+c \frac{\partial w}{\partial t}=f(x, t)
$$

where $c$ is the damping constant for the beam in a specific environment and is typically found experimentally. For a beam clamped at each end, the boundary conditions are given as

$$
\begin{gathered}
w(0, t)=0 \\
\frac{\partial w(0, t)}{\partial x}=0 \\
w(L, t)=0 \\
\frac{\partial w(L, t)}{\partial x}=0
\end{gathered}
$$

Eq. 3.33-3.37 give the Euler-Bernoulli beam model adjusted for axial stretching considerations. If the boundary conditions of the beam are considered completely immovable (which is the case for a clamped-clamped beam), an axial membrane force 
in the beam is created as the beam's length increases due to transverse deflection. This membrane force is given as

$$
N(x, t)=E A\left(\frac{u_{L}(t)-u_{0}(t)}{L}+\frac{1}{2 L} \int_{0}^{L}\left(\frac{\partial w}{\partial x}\right)^{2} d x\right)
$$

where $u_{L}$ is the axial deformation at $x=L$ and $u_{0}$ is the axial deformation at $x=0$. Assuming both are equal to zero and substituting Eq. 3.38 into Eq. 3.33 gives

$$
E I \frac{\partial^{4} w}{\partial x^{4}}+\rho A \frac{\partial^{2} w}{\partial t^{2}}-\frac{E A}{2 L}\left(\int_{0}^{L}\left(\frac{\partial w}{\partial x}\right)^{2} d x\right) \frac{\partial^{2} w}{\partial x^{2}}+c \frac{\partial w}{\partial t}=f(x, t)
$$

Eq. 3.39 along with the boundary conditions provided in Eq. 3.34-3.37 give the complete beam model accounting for axial stretching.

\subsubsection{Multiple Mode Approximation}

Reintroducing the variable, $\zeta=\frac{x}{L}$, and a periodic forcing term, Eq. 3.39 then becomes

$$
E I \frac{\partial^{4} w}{\partial \zeta^{4}}+\rho A \frac{\partial^{2} w}{\partial t^{2}}-\frac{E A}{2 L}\left(\int_{0}^{L}\left(\frac{\partial w}{\partial \zeta}\right)^{2} d \zeta\right) \frac{\partial^{2} w}{\partial \zeta^{2}}+c \frac{\partial w}{\partial t}=F \sin (\Omega t)
$$

were $F$ is the forcing amplitude and $\Omega$ is the forcing frequency. Following the Galerkin discretization technique for multiple modes, one assumes that the solution of Eq. 3.40 takes the form

$$
w(\zeta, t)=\sum_{n=1}^{N} \phi_{n}(\zeta) q_{n}(t)
$$

Substituting Eq. 3.41 into Eq. 3.40 gives 


$$
\begin{aligned}
& E I \sum_{n=1}^{N}\left[q_{n} \frac{d^{4} \phi_{n}}{d \zeta^{4}}\right]+\rho A \sum_{n=1}^{N}\left[\phi_{n} \frac{d^{2} q_{n}}{d t^{2}}\right]-\frac{E A}{2 L} \sum_{n=1}^{N}\left[\left(\int_{0}^{1}\left(q_{n} \frac{d \phi_{n}}{d \zeta}\right)^{2} d \zeta\right) \frac{d^{2} \phi_{n}}{d \zeta^{2}}\right] \\
& +c \sum_{n=1}^{N} \phi_{n} \frac{d q_{n}}{d t}=F \sin (\Omega t)
\end{aligned}
$$

Using shorthand notation for spatial and temporal derivatives, the Galerkin method is implemented by multiplying Eq. 3.42 by $\phi_{m}$ and integrating with respect to $\zeta$ from 0 to 1 . This process gives

$$
\begin{gathered}
E I \sum_{n=1}^{N} q_{n} \int_{0}^{1} \phi_{m} \phi_{n}^{\prime \prime \prime \prime} d \zeta+\rho A \sum_{n=1}^{N} \ddot{q}_{n} \int_{0}^{1} \phi_{n} \phi_{m} d \zeta+c \sum_{n=1}^{N} \dot{q}_{n} \int_{0}^{1} \phi_{n} \phi_{m} d \zeta \\
-\frac{E A}{2 L} \int_{0}^{1} \phi_{m}\left[\left(\int_{0}^{1}\left(\sum_{i=1}^{N} q_{i} \phi_{i}^{\prime}\right)^{2} d \zeta\right) \sum_{n=1}^{N} \phi_{n}^{\prime} q_{n}\right]=F \sin (\Omega t) \int_{0}^{1} \phi_{m} d \zeta
\end{gathered}
$$

Note the change of summation index in the fourth term in Eq. 3.43. When the Galerkin method is applied to nonlinear terms, each approximation of the total response (Eq. 3.41) needs to have a separate set of indices. This is done to capture all modal cross-coupling effects (Younis (2011) and Wagg and Neild (2014)).

$$
\begin{array}{r}
E I \sum_{n=1}^{N} q_{n} \int_{0}^{1} \phi_{m} \phi_{n}^{\prime \prime \prime \prime} d \zeta+\rho A \sum_{n=1}^{N} \ddot{q_{n}} \int_{0}^{1} \phi_{n} \phi_{m} d \zeta+c \sum_{n=1}^{N} \dot{q}_{n} \int_{0}^{1} \phi_{n} \phi_{m} d \zeta \\
-\frac{E A}{2 L} \int_{0}^{1} \phi_{m}\left[\left(\int_{0}^{1}\left(\sum_{i=1}^{N} q_{i} \phi_{i}^{\prime}\right)\left(\sum_{j=1}^{N} q_{j} \phi_{j}^{\prime}\right) d \zeta\right) \sum_{n=1}^{N} \phi_{n}^{\prime \prime} q_{n}\right]=F \sin (\Omega t) \int_{0}^{1} \phi_{m} d \zeta
\end{array}
$$

The first three terms in Eq. 3.44 can be reduced via orthogonality of mode shapes when $n=m$. Therefore we perform the reduction and adjust the subscripts on the first three terms. Furthermore, we expand the fourth term in Eq. 3.44 and include 
additional summation indices (due to nonlinearity) giving

$$
\begin{array}{r}
\rho A \ddot{q_{m}}+E I k_{m}^{2} q_{m}+c \dot{q_{m}}-\frac{E A}{2 L} \int_{0}^{1} \phi_{m}\left[\left(\int_{0}^{1}\left(\sum_{i=1}^{N} q_{i} \phi_{i}^{\prime}\right)\left(\sum_{j=1}^{N} q_{j} \phi_{j}^{\prime}\right) d \zeta\right) \sum_{n=1}^{N} \phi_{n}^{\prime \prime} q_{n}\right] \\
=F \sin (\Omega t) \int_{0}^{1} \phi_{m} d \zeta
\end{array}
$$

Finally, after further simplification, the multiple mode discretization is presented as

$$
\rho A \ddot{q_{m}}+E I k_{m}^{2} q_{m}+c \dot{q_{m}}+\frac{E A}{2 L} \sum_{m, n, i, j=1}^{N} A_{m n i j} q_{n} q_{i} q_{j}=B_{m} F \sin (\Omega t)
$$

where

$$
A_{m n i j}=-\int_{0}^{1} \phi_{m} \phi_{n}^{\prime \prime} d \zeta \int_{0}^{1} \phi_{i}^{\prime} \phi_{j}^{\prime} d \zeta
$$

and

$$
B_{m}=\int_{0}^{1} \phi_{m} d \zeta
$$

Equation 3.46 is the discretized beam model where the stiffness is affected by axial stretching due to large transverse deflection. We see cubic nonlinear terms that are a direct effect of the axial stretching. The response is in modal coordinates and can be simulated via numerical integration or perturbation methods. The full response of the beam in temporal and spatial coordinates can be achieved by inserting Eq. 3.46 into Eq. 3.41. 


\subsection{Buckled Beam Model}

Consider the beam shown in Fig. 3.4 that has been compressed by an axial load, $P$, past its first critical buckling load. Investigating a small section of the beam, $d x$, Fig. 3.5 gives the free body diagram of this buckled configuration.

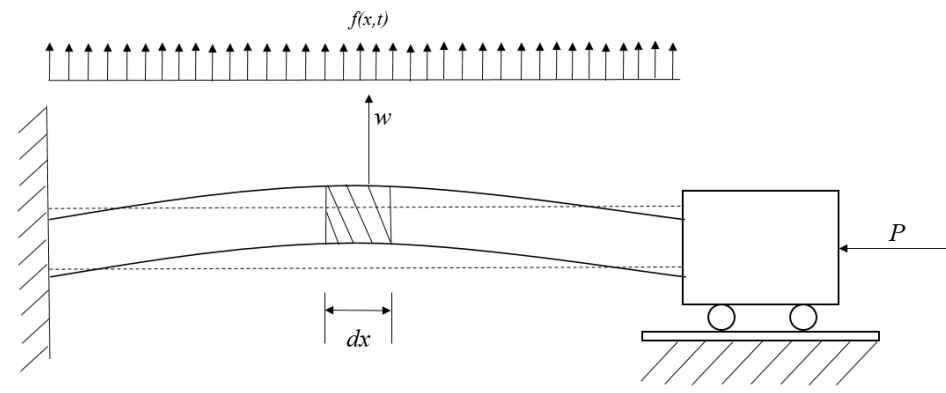

Figure 3.4: Buckled Beam Kinematic Diagram

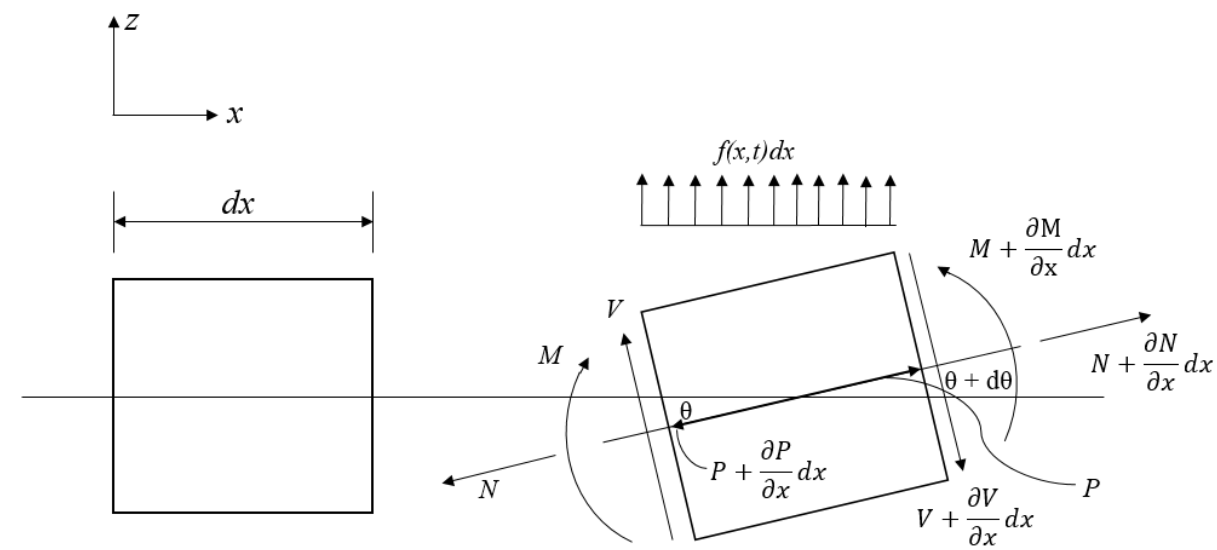

Figure 3.5: Buckled Beam Free Body Diagram

Note the addition of an internal axial force, $P$, which is due to the axial compressive load. Using the small angle approximation and the same variable substitution as before (i.e. $\theta+d \theta=\frac{\partial w}{\partial x}+\frac{\partial^{2} w}{\partial x^{2}}$ ), we balance forces in the positive $z$ direction and 
equate them to an inertial term, giving

$$
\begin{gathered}
-\left(V+\frac{\partial V}{\partial x} d x\right)+V+\left(N+\frac{\partial N}{\partial x} d x\right)\left(\frac{\partial w}{\partial x}+\frac{\partial^{2} w}{\partial x^{2}} d x\right)-N \frac{\partial w}{\partial x} \\
-\left(P+\frac{\partial P}{\partial x} d x\right)\left(\frac{\partial w}{\partial x}+\frac{\partial^{2} w}{\partial x^{2}} d x\right)+P \frac{\partial w}{\partial x}+f(x, t)=\rho A \frac{\partial^{2} w}{\partial t^{2}}
\end{gathered}
$$

Simplifying and ignoring terms of order $(d x)^{2}$ gives

$$
-\frac{\partial V}{\partial x}+N \frac{\partial^{2} w}{\partial x^{2}}-P \frac{\partial^{2} w}{\partial x^{2}}+f(x, t)=\rho A \frac{\partial^{2} w(x, t)}{\partial t^{2}}
$$

Summing the moments about the center of the element and ignoring rotary inertia (typically of negligible effect) gives

$$
-\left(M+\frac{\partial M}{\partial x} d x\right)+M+\left(V+\frac{\partial V}{\partial x} d x\right) \frac{d x}{2}+V \frac{d x}{2}=0
$$

Simplifying and ignoring terms of order $(d x)^{2}$ gives

$$
V(x, t)=\frac{\partial M}{\partial x}
$$

Inserting Eq. 3.52 into Eq. 3.50 and rearranging gives

$$
-\frac{\partial^{2} M}{\partial x^{2}}+N \frac{\partial^{2} w}{\partial x^{2}}-P \frac{\partial^{2} w}{\partial x^{2}}+f(x, t)=\rho A \frac{\partial^{2} w}{\partial t^{2}}
$$

From elementary theory of beam bending, the relationship between beam deflection and bending moment is given as

$$
M(x, t)=E I \frac{\partial^{2} w(x, t)}{\partial x^{2}}
$$

Substituting Eq. 3.54 into Eq. 3.53 and rearranging gives 


$$
\frac{\partial^{2}}{\partial x^{2}}\left[E I \frac{\partial^{2} w}{\partial x^{2}}\right]+\rho A \frac{\partial^{2} w}{\partial t^{2}}-N \frac{\partial^{2} w}{\partial x^{2}}+P \frac{\partial^{2} w}{\partial x^{2}}=f(x, t)
$$

Where $N(x, t)$ is given as

$$
N(x, t)=E A\left(\frac{u_{L}(t)-u_{0}(t)}{L}+\frac{1}{2 L} \int_{0}^{L}\left(\frac{\partial w}{\partial x}\right)^{2} d x\right)
$$

Again, assuming the axial deformation at $x=L\left(u_{L}\right)$, and $x=0\left(u_{0}\right)$ are both zero, and introducing a velocity dependent damping term, Eq. 3.55 becomes

$$
E I \frac{\partial^{4} w}{\partial x^{4}}+\rho A \frac{\partial^{2} w}{\partial t^{2}}-\frac{E A}{2 L}\left(\int_{0}^{L}\left(\frac{\partial w}{\partial x}\right)^{2} d x\right) \frac{\partial^{2} w}{\partial x^{2}}+P \frac{\partial^{2} w}{\partial x^{2}}+c \frac{\partial w}{\partial t}=f(x, t)
$$

For a beam clamped at each end, the boundary conditions are given

$$
\begin{gathered}
w(0, t)=0 \\
\frac{\partial w(0, t)}{\partial x}=0 \\
w(L, t)=0 \\
\frac{\partial w(L, t)}{\partial x}=0
\end{gathered}
$$

\subsubsection{Buckled Beam Static Mode Shapes}

At this point, we temporarily introduce the following dimensionless variables for brevity: $\hat{w}=\frac{w}{r}, \zeta=\frac{x}{L}, \hat{P}=\frac{P L^{2}}{E I}, \hat{c}=\frac{c L^{2}}{\sqrt{(\rho A E I)}}, \hat{t}=t \sqrt{\frac{E I}{\rho A L^{4}}}, \hat{F}=\frac{F L^{4}}{\sqrt{r E I}}$, and $\hat{\Omega}=\Omega \sqrt{\frac{\rho A L^{4}}{E I}}$, where $r=\sqrt{I / A}$ is the radius of gyration of the cross-section. Eq. 
3.57 thus becomes

$$
\hat{w}^{\prime \prime \prime \prime}+\ddot{\hat{w}}-\hat{w}^{\prime \prime} \frac{1}{2} \int_{0}^{1} \hat{w}^{2} d \zeta+\hat{P} \hat{w}^{\prime \prime}+\hat{c} \dot{\hat{w}}=\hat{F} \sin (\hat{\Omega} \hat{t})
$$

Where the overdot indicates temporal and prime indicates spatial derivative. The boundary conditions are given as

$$
\begin{aligned}
& \hat{w}(0, t)=0 \\
& \hat{w}^{\prime}(0, t)=0 \\
& \hat{w}(\zeta, t)=0 \\
& \hat{w}^{\prime}(\zeta, t)=0
\end{aligned}
$$

To derive the static buckled mode shapes, the time dependent terms are dropped from Eq. 3.62, giving

$$
\Psi_{B}^{\prime \prime \prime \prime}-\Psi_{B}^{\prime \prime} \frac{1}{2} \int_{0}^{1} \Psi_{B}^{\prime 2} d \zeta+\hat{P} \Psi_{B}^{\prime \prime}=0
$$

where $\Psi_{B}$ is the static buckling configuration. Furthermore, the definite integral in Eq. 3.67 is a constant for any function $\Psi_{B}$, therefore we let

$$
Q=\frac{1}{2} \int_{0}^{1} \Psi_{B}^{\prime 2} d \zeta
$$

where Q is a constant. Eq. 3.67 reduces to

$$
\Psi_{B}^{\prime \prime \prime \prime}+\lambda^{2} \Psi_{B}^{\prime \prime}=0
$$


where $\lambda=\sqrt{(P-Q)}$. The solution of Eq. 3.69 is given as

$$
\Psi_{B}(\zeta)=c_{1}+c_{2} \zeta+c_{3} \cos (\lambda \zeta)+c_{4} \sin (\lambda \zeta)
$$

where $c_{1}, c_{2}, c_{3}, c_{4}$ are constants. Using the boundary conditions described by Eq. 3.63 - Eq. 3.66, results in four equations

$$
\begin{gathered}
c_{1}+c_{3}=0 \\
c_{2}+\lambda c_{4}=0 \\
c_{1}+c_{2}+c_{3} \cos (\lambda)+c_{4} \sin (\lambda)=0 \\
c_{2}-\lambda c_{3} \sin (\lambda)+c_{4} \lambda \cos (\lambda)=0
\end{gathered}
$$

This system of equations represents an eigenvalue problem in which the eigenvalues are the Euler critical buckling loads and the eigenvectors are the buckled mode shapes. From this process the static buckled mode shapes are found to be

$$
\Psi_{B}= \begin{cases}\frac{b}{2}\left(1-\cos \left(\lambda_{n} \zeta\right)\right), & \text { if } n \text { is odd } \\ \frac{b}{2}\left(1-2 \zeta-\cos \left(\lambda_{n} \zeta\right)+\frac{2}{\lambda_{n}} \sin \left(\lambda_{n} \zeta\right)\right) & \text { if } n \text { is even }\end{cases}
$$

where $b$ is the static nondimensional rise in at the midspan of the beam (ratio of physical static deflection to beam thickness). In terms of the external compressive load, this nondimensional rise is

$$
b=\sqrt{\frac{4\left(P-P_{c}\right)}{\pi^{2}}}
$$

where $P_{c}$ is the nondimensional critical buckling load and is given as $\lambda_{n}^{2}$ where values for $\lambda_{n}$ are shown in Table 3.2.

For the remainder of the study, we will only consider the beam buckled past the 


\begin{tabular}{|cc|}
\hline $\mathbf{n}$ & $\lambda$ \\
\hline 1 & 6.2832 \\
2 & 8.9852 \\
3 & 12.5664 \\
\hline
\end{tabular}

Table 3.2: Non-dimensional Critical Buckling Load Constants

first critical buckling load and before the second buckling load.

Before we follow the multiple mode discretization process, we must adjust the partial differential equations of motion to account for vibration about the static buckled configuration.

\subsubsection{Vibration About The Buckled Configuration}

In order to derive an expression for the total response of the buckled beam we assume the solution takes the form

$$
\hat{w}(\zeta, t)=\Psi_{B}(\zeta)+v(\zeta, t)
$$

Where $v(\zeta, t)$ is the vibration response about the buckled mode shape. Substituting Eq. 3.77 into Eq. 3.62 gives

$\left(v^{\prime \prime \prime \prime}+\Psi_{B}^{\prime \prime \prime \prime}\right)+\left(\ddot{v}+\ddot{\Psi}_{B}\right)-\left(v^{\prime \prime}+\Psi_{B}^{\prime \prime}\right) \frac{1}{2} \int_{0}^{1}\left(v^{\prime}+\Psi_{B}^{\prime}\right)^{2} d \zeta+\hat{P}\left(v^{\prime \prime}+\Psi_{B}^{\prime \prime}\right)+\hat{c}\left(\dot{v}+\dot{\Psi}_{B}\right)=\hat{F} \sin (\hat{\Omega} \hat{t})$

Making use of Eq. 3.69, Eq. 3.78 becomes

$v^{\prime \prime \prime \prime}+\ddot{v}+\hat{P} v^{\prime \prime}+\hat{c} \dot{v}-\Psi_{B}^{\prime \prime} \int_{0}^{1} \Psi_{B}^{\prime} v^{\prime} d \zeta-\frac{1}{2} \Psi_{B}^{\prime \prime} \int_{0}^{1} v^{\prime 2} d \zeta-v^{\prime \prime} \int_{0}^{1} \Psi_{B}^{\prime} v^{\prime} d \zeta-\frac{1}{2} v^{\prime \prime} \int_{0}^{1} v^{\prime 2} d \zeta=\hat{F} \sin (\hat{\Omega} \hat{t})$

Eq. 3.79 gives the full equation of motion for the beam vibrating about any given 
buckled configuration. To simulate the response, we continue with the multiple mode discretization method.

\subsubsection{Multiple Mode Approximation}

Following the Galerkin discretization technique for multiple modes, one assumes that the solution of Eq. 3.79 takes the form

$$
v(\zeta, t)=\sum_{n=1}^{N} \phi_{n}(\zeta) q_{n}(t)
$$

Following the steps presented in the nonlinear straight beam discretization, the multiple mode discretization for the buckled beam results in

$$
\begin{array}{r}
\rho A \ddot{q}_{m}+c \dot{q}_{m}+P E I q_{m}+\frac{E A}{2 L}\left[\sum_{m, n, i=1}^{N} A_{m n i} q_{n} q_{i}+\right. \\
\left.\sum_{m, n, i, j=1}^{N} B_{m n i j} q_{n} q_{i} q_{j}\right]=C_{m} F \sin (\Omega t)
\end{array}
$$

where

$$
\begin{gathered}
A_{m n i}=\left(\int_{0}^{1} \phi_{n}^{\prime} \phi_{m}^{\prime} d \zeta\right)\left(\int_{0}^{1} \phi_{i}^{\prime} \Psi_{B}^{\prime} d \zeta\right)+\left(\int_{0}^{1} \phi_{n}^{\prime} \phi_{i}^{\prime} d \zeta\right)\left(\int_{0}^{1} \Psi_{B}^{\prime \prime} \phi_{m} d \zeta\right) \\
B_{m n i j}=-\frac{1}{2}\left(\int_{0}^{1} \phi_{n}^{\prime \prime} \phi_{m} d \zeta\right)\left(\int_{0}^{1} \phi_{i}^{\prime} \phi_{j}^{\prime} d \zeta\right)
\end{gathered}
$$

and

$$
C_{m}=\int_{0}^{1} \phi_{m} d \zeta
$$

Eq. 3.81 represents a set of coupled nonlinear ordinary differential equations with 
both cubic and quadratic nonlinearities. The dynamic response can be simulated via numerical integration or perturbation methods.

\subsection{Buckled Beam Model With Piezoelectric Excitation}

In this section, the nonlinear buckled beam model will be modified to include hysteretic forcing via piezoelectric patches. The added mass due to the piezoelectric patches will be neglected in this study. We will first describe the physical mechanism that causes piezoelectric actuation and then model the hysteretic behavior.

In general, the direct piezoelectric effect describes the phenomenon that occurs when a piezoelectric material is subjected to a mechanical stress. When under stress, the material responds by producing an electric displacement. Specifically, the direct effect can be expressed by

$$
D=d \sigma+e^{T} E_{f}
$$

Where $D$ is the electric displacement, $d$ is the piezoelectric constants, $\sigma$ is the mechanical stress, $e^{T}$ is the permittivity of the medium, and $E_{f}$ is the electric field. The direct piezoelectric effect is typically employed in piezoelectric sensor design as is converts mechanical stress into an electric displacement. For use as actuators, the converse piezoelectric effect is used to convert an applied electric field into mechanical strain/displacement. The converse effect is governed by the following relation:

$$
\epsilon=s^{E} \sigma+d E_{f}
$$

where $\epsilon$ is the mechanical strain and $s^{E}$ is the compliance of the medium. As shown in Fig. 3.6, when voltage is applied to a piezoelectric actuator in the transverse ( $z$ or (3)) direction, the element generates mechanical strain in the longitudinal ( $x$ or (1)) direction. This effect is known as $d_{31}$ actuation. 


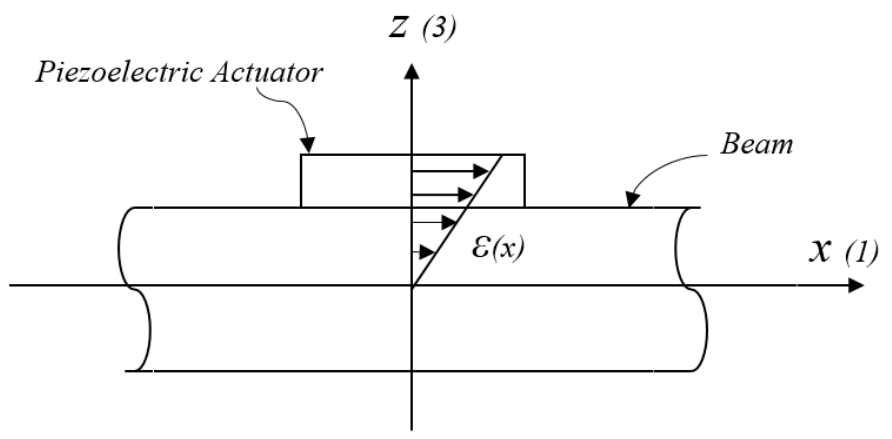

Figure 3.6: Piezoelectric Actuator Cutaway

When perfectly bonded to the beam surface, the mechanical strain in the piezoelectric element generates a bending moment about the neutral axis of the piezo-beam system. Following Rathi and Khan (2012), the resulting distributed transverse force generated by the piezoelectric element is given as

$$
F_{P}=\frac{E_{P} d_{31} w_{P} \bar{y} V}{L_{P}}
$$

where $E_{P}$ is the piezoelectric elastic modulus, $d_{31}$ is the piezoelectric constant in the 3-1 direction, $w_{P}$ is the width of the piezoelectric patch, $L_{p}$ is the length of the piezoelectric patch, $V$ is the applied voltage, and $\bar{y}$ is the distance from the neutral axis of the beam to the neutral axis of the piezoelectric element. It can be seen that with an alternating voltage, a piezoelectric element can generate a periodic transverse force on the beam. When $m$ number of patches are used, and are closely spaced along the beam, the force generated along the beam is simply the sum of the forces generated by each element (Moheimani and Fleming (2006)):

$$
F_{P}(x, t)=\sum_{i=1}^{m} F_{P_{i}}(x, t)
$$

While Eq. 3.87 describes the amplitude of the force applied by a piezoelectric patch, it assumes a linear actuation profile. This is not always the case, however, as piezoelectric actuators have shown strong hysteresis qualities in the actuation profile. 
As such, we follow a phenomenological approach to modeling the hysteresis behavior. Following the work of Low and Guo (1995), we define the relationship between an input excitation variable, $x$, and an output response variable, $z$ as

$$
\dot{z}=\alpha \dot{x}-\beta|\dot{x}| z-\gamma \dot{x}|z|
$$

Where $\alpha$ controls the restoring hysteresis force, while $\beta$ and $\gamma$ control the profile shape of the hysteresis loop. For demonstration purposes, Fig. 3.7 shows several hysteresis curves for different combinations of shape control parameters with (a): $\alpha$ $=1.0, \beta=0.5, \gamma=0.1 ;(\mathrm{b}): \alpha=1.0, \beta=0.1, \gamma=0.9 ;(\mathrm{c}): \alpha=1.0, \beta=0.5, \gamma=$ $-0.5 ;(d): \alpha=1.0, \beta=0.25, \gamma=-0.5 ;$

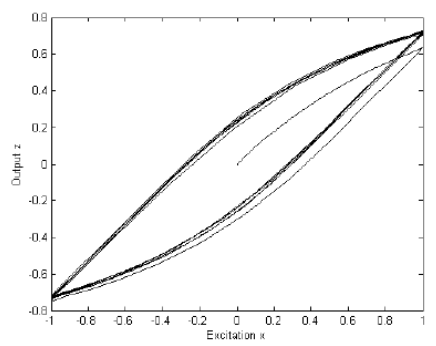

(a)

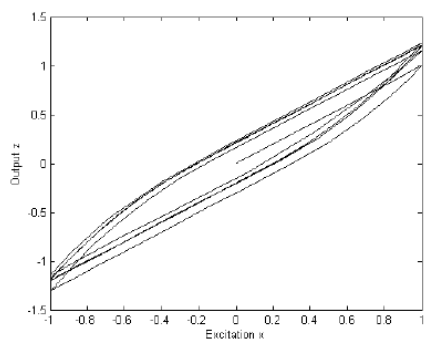

(c)

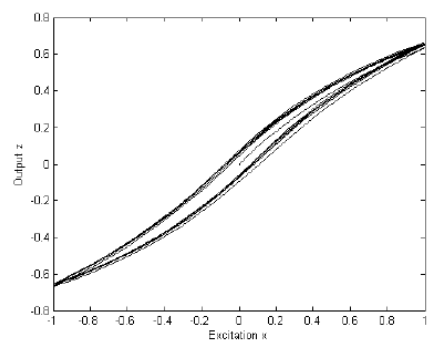

(b)

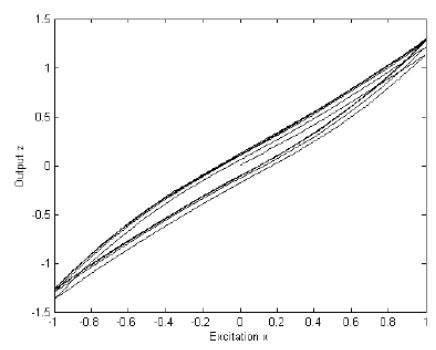

(d)

Figure 3.7: Various Hysteresis Models

Figure 3.7 shows how the shape and magnitude of the hysteresis loop can be altered and adjusted with different parameter values. More importantly, however, we observe that the hysteresis model is a trajectory and has a transient initial response which eventually settles to a steady-state periodic response. As we aim to simulate 
harmonic responses of the beam models, the initial value of the hysteresis trajectory must chosen to ensure immediate periodicity without any transient behavior. This consideration is discussed in detail in Chapter VI.

Adjusting the hysteresis model to account for physical piezoelectric properties, the input excitation is changed to voltage, $V$, giving

$$
\dot{z}=\alpha d_{31} \dot{V}-\beta|\dot{V}| z-\gamma \dot{V}|z|
$$

where $d_{31}$ is the piezoelectric constant in the 3-1 direction. The total hysteresis output force generated by the piezoelectric element is given as

$$
F_{P}=k\left(d_{31} V-z\right)
$$

where $k$ is the axial stiffness of the piezoelectric element, given by

$$
k=\frac{E_{P} w_{P} \bar{y}}{L_{P}}
$$

Consideration will be made in choosing piezoelectric parameter values that give rise to a hysteresis forcing function that matches well with published experimental data.

In addition to the hysteresis forcing profile from the piezoelectric elements, the elements add mass and stiffness to the beam model. As such, we will add the stiffness terms for the beam and piezoelectric elements together $(E I)$ as well as the mass per unit length terms terms $(\rho A)$. This combination is in agreement with the approach taken by Donoso and Sigmund (2009) for modeling multilayer piezoelectric bimorphs. Returning to the nonlinear buckled beam model, we will apply a sinusoidal voltage to the piezoelectric element so the total model becomes 


$$
\begin{array}{r}
\rho A \ddot{q}_{m}+c \dot{q}_{m}+P E I q_{m}+\frac{E A}{2 L}\left[\sum_{m, n, i=1}^{N} A_{m n i} q_{n} q_{i}+\right. \\
\left.\sum_{m, n, i, j=1}^{N} B_{m n i j} q_{n} q_{i} q_{j}\right]=C_{m} k\left(d_{31} V \sin (\Omega t)-z\right)
\end{array}
$$

Eq. 3.93 represents the complete nonlinear piezoelectrically-driven buckled beam model, accounting for hysteresis forcing. This model along with the buckled beam model with generic harmonic excitation is used in this work. 


\section{CHAPTER IV}

\section{Periodic Response}

\subsection{Introduction}

Many nonlinear systems are operated under the presence of harmonic excitation with intention to induce a periodic system response. When a nonlinear system is forced harmonically, the response of the system can show high sensitivity to the forcing configuration. In some cases, there may be several possible periodic solutions that exist for a single forcing configuration. Furthermore, for some forcing configurations, a highly nonlinear system can behave in a linear fashion while for other configurations, it can exhibit strong nonlinearity. Therefore, in order to fully understand the dynamics of a nonlinear system, it is important to (1) predict and achieve periodic responses, and (2) determine their dynamic stability.

There are many techniques to predict and calculate periodic orbits of nonlinear systems. Despite the diversity of the tools used in industry, the techniques typically fall into one of two categories. The first is an analytical approach and the second is a numerical one. Typical analytical techniques employ approximation methods to the differential equations and attempt to develop algebraic expressions for the periodic orbits. One such method is called the harmonic balance technique in which one assumes a solution in the form of a harmonic series (sometimes truncated after one harmonic term) and collects like terms on each side of the equations. While harmonic 
balance is a common tool used to calculate periodic orbits, it falls short as it tends to exhibit large error when the response contains strong nonlinearities (Genesio and Tesi (1992)). Mitigating this by adding more harmonic terms is often beneficial but at the cost of computational time and resources. The "method of multiple scales" and the "method of averaging" are two other analytical techniques in which perturbation analysis is employed. In these methods, one attempts to find an asymptotic linearization of the nonlinear solution. A number of applications of these methods have proven successful Sze et al. (2005), however in the case of complicated forcing or strong nonlinearities, the analytical techniques can be difficult to implement.

The second category of predicting periodic orbits is that of numerical methods. In these methods, one deals directly with the nonlinear equations and performs direct time integration to search for a solution. Without the requirement of closed formed equations of motion, this direct technique can be powerful. Basically, a system is directly integrated until it settles to a semi-periodic response. A zero-finding method is then employed to iterate the initial conditions and forcing frequency to step closer to a perfectly-periodic orbit. Among the zero-finding algorithms, the Newton-Raphson correction methods is most prevalent as it converges to a solution quadratically. This approach is typically started with an initial guess and is subsequently corrected to satisfy a convergence criteria. Using the Newton-Raphson method to attain periodic orbits is called the shooting method. Extending from the shooting method, a parameter of interest can then be varied to predict and compute a branch of solutions. This prediction method is called continuation. For this study, the shooting method combined with a continuation method is employed as it allows for parametric studies and can handle complicated systems more efficiently than analytical techniques. The derivations in this chapter follow the work of Peeters et al. (2008), and Sracic and Allen (2011). 


\subsection{The Newton-Raphson Method}

Consider a function $f(x)$ with a zero crossing at $x^{*}$ such that $f\left(x^{*}\right)=0$. The Newton-Raphson method makes use of a local tangent, $f^{\prime}\left(x^{i}\right)$, and extrapolates that tangent's zero crossing. With $x^{i}$ representing the previous guess for the method, the next iteration, $x^{i+1}$ is found as

$$
x^{i+1}=x^{i}-\frac{f\left(x^{i}\right)}{f^{\prime}\left(x^{i}\right)}
$$

By choosing a starting guess sufficiently close to the zero crossing, the algorithm is repeated until a convergence criteria is met. The Newton-Raphson method can also be used to find a zero of a multi-dimensional function, $\boldsymbol{f}$. A set of linear equations is formed

$$
\boldsymbol{D} \boldsymbol{f}\left(\boldsymbol{x}^{i}\right) \Delta \boldsymbol{x}=-\boldsymbol{f}\left(\boldsymbol{x}^{i}\right)
$$

and the next iteration guess is generated by $\boldsymbol{x}^{i+1}=\boldsymbol{x}^{i}+\Delta \boldsymbol{x}$ where $\boldsymbol{D} \boldsymbol{f}$ is the Jacobian matrix, and $\boldsymbol{x}$ is a vector of independent variables. The Newton-Raphson method is a gradient-based method because it employs the local gradient to select the next guess. Gradient-based zero finding methods can have relatively high convergence rates, however careful selection of starting guesses is needed as the methods are local (not global) solvers.

\subsection{The Shooting Method}

Consider a time varying system with response vector $x(t)$ periodically forced with frequency $\Omega$. After a certain amount of time, the transient response of the system will decay and steady state solution will remain. Let $x_{T}$ be the response vector at

the end of one period of forcing $\left(T=\frac{2 \pi}{\Omega}\right)$ and $x_{0}$ be the response vector at the initial 
state (i.e. the initial condition vector). This is best illustrated for state variables $x(t)$ and $\dot{x}(t)$ as shown in Fig. 4.1.
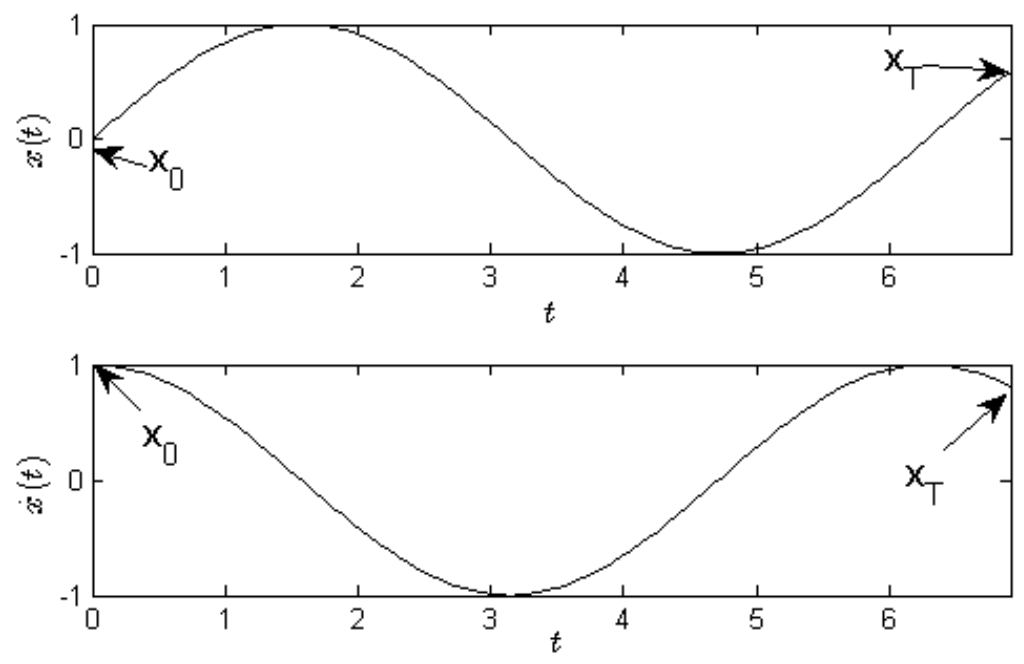

Figure 4.1: Response After One Forcing Period

The difference between vector $x_{T}$ and vector $x_{0}$ defines the shooting function $H\left(x_{0}, T\right)$

$$
H\left(\boldsymbol{x}_{\mathbf{0}}, T\right) \equiv \boldsymbol{x}_{\boldsymbol{T}}-\boldsymbol{x}_{\mathbf{0}}
$$

The goal of the shooting method is to solve the two-point boundary value problem and determine values for the initial state vector, $x_{0}$ and the period, $T$, such that $H\left(x_{0}, T\right)=0$

We begin by expressing the shooting function as a Taylor Series expansion about $\boldsymbol{x}_{\mathbf{0}}+\Delta \boldsymbol{x}_{\mathbf{0}}$ and $T+\Delta T$ :

$$
H\left(\boldsymbol{x}_{\mathbf{0}}, T\right)+\frac{\partial H}{\partial \boldsymbol{x}_{\mathbf{0}}} \Delta \boldsymbol{x}_{\mathbf{0}}+\frac{\partial H}{\partial T} \Delta T=0
$$

Adjusting Eq. 4.4, we obtain the multi-dimensional linear system in the form of the Newton-Raphson method: 


$$
\left[\begin{array}{ll}
\frac{\partial H}{\partial \boldsymbol{x}_{\mathbf{0}}} & \frac{\partial H}{\partial T}
\end{array}\right]\left[\begin{array}{c}
\Delta \boldsymbol{x}_{\mathbf{0}} \\
\Delta T
\end{array}\right]=\left[-H\left(\boldsymbol{x}_{\mathbf{0}}, T\right)\right]
$$

Using the Newton-Raphson algorithm, $x_{0}$ and $T$ are adjusted by $\Delta x_{0}$ and $\Delta T$, respectively, until the shooting function is below an acceptable convergence value. The outcome of this method is a periodic solution (also called orbit or trajectory). To illustrate the method, Fig. 4.2 shows state variable $\dot{x}(t)$ against state variable $x(t)$ before shooting method. After successful completion of the shooting method, Fig. 4.3 is attained and the solution is periodic.

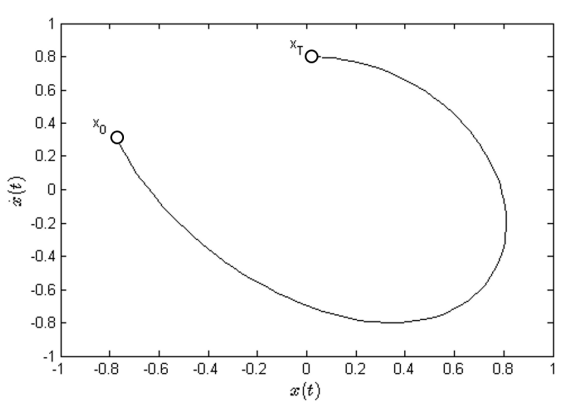

Figure 4.2: Before Shooting Method

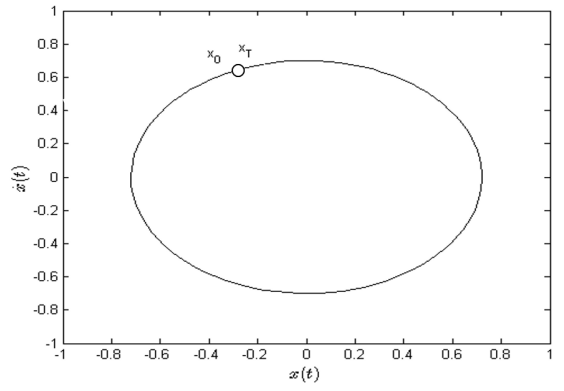

Figure 4.3: After Shooting Method

In order to evolve the periodic solutions across a range of forcing frequencies, a continuation method is needed to predict the location of other periodic solutions in $x_{0^{-}}$ $\Omega$ space. Continuation methods typically use the shooting method as an intermediate step of the algorithms. Two types of continuation methods exist: sequential and arclength continuation. Each are discussed next.

\subsection{Sequential Continuation}

The shooting method is able to refine a guess into a periodic solution but can not find a periodic solution out of thin air. However by finding a periodic solution (via shooting method) and perturbing the forcing frequency by a small enough value, an additional implementation of the shooting method should be able to refine to a 
new periodic solution at that new frequency. If this process is continued across a frequency range of interest, a frequency response curve can be generated by simply plotting the response amplitude (A) after each frequency advancement and shooting method refinement. In a general sense, however, sequential continuation allows for the variation of a system parameter and measurement of any metric, after a periodic solution is attained. Figure 4.4 shows the sequential continuation method varying a system parameter (the forcing frequency) and plotting the subsequent response metric (the response amplitude, in this example), after periodicity is achieved

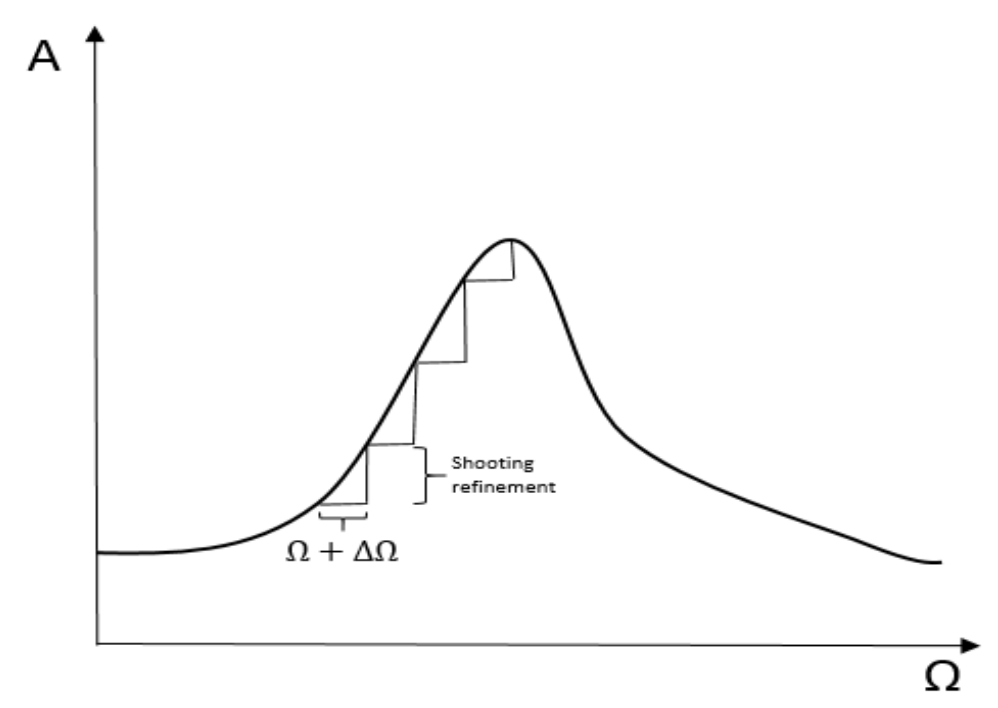

Figure 4.4: Sequential Continuation

Sequential continuation is a suitable technique for producing frequency response curves for linear and weakly nonlinear systems. For strongly nonlinear systems, however, sequential continuation has a major drawback in that the frequency advancement direction and step size is fixed. This drawback is due to the fact that in strongly nonlinear systems, jump phenomenon as in Fig. 4.5 is present and can most easily be visualized in nonlinear frequency response plots in which a single given forcing frequency $\Omega$, gives rise to more than one periodic solution. As the forcing frequency is swept along the range of interest, sequential continuation cannot handle doubling back toward frequencies of multiple solutions. 


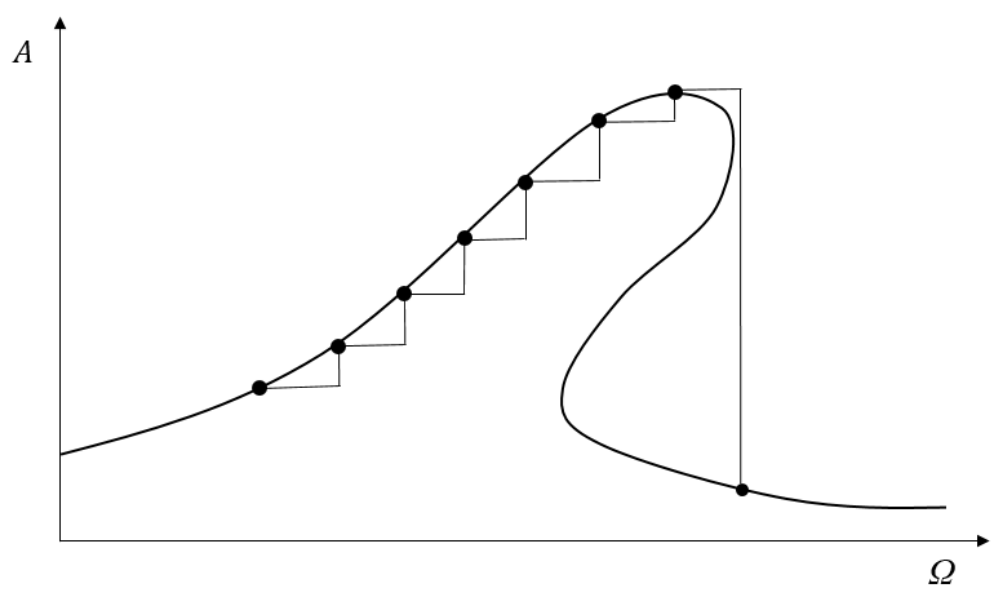

Figure 4.5: Sequential Continuation for Strongly Nonlinear System

A way to mitigate this shortfall of sequential continuation is to alter the algorithm to allow for varying frequency step sizes and directions. In general, this type of algorithm is called a predictor-corrector technique and with its application to nonlinear systems it is called arc length continuation.

\subsection{Arc-Length Continuation}

The arc-length continuation method is primarily based on a predictor step that is tangent to the current periodic solution branch and subsequent corrector steps that forces the algorithm to converge back to a periodic solution at a new frequency. The method can round turning points of nonlinear frequency response functions and therefore can double back to reevaluate previous frequencies and find different periodic solutions. The algorithm begins by using the shooting method to calculate a periodic solution at a starting frequency value. This is typically performed at a frequency that has relatively low energy and is in the linear regime of the nonlinear system, if possible. After the starting periodic solution is found, a predictor step is implemented 
advances the forcing frequency as well as the initial state vector in a direction that is tangent to the current solution branch. The predictor step is given by the system

$$
\left[\begin{array}{ll}
\frac{\partial H}{\partial \boldsymbol{x}_{\mathbf{0}}} & \frac{\partial H}{\partial T}
\end{array}\right]\left[\begin{array}{c}
\boldsymbol{P}_{\boldsymbol{x}_{\mathbf{0}}} \\
P_{T}
\end{array}\right]=\mathbf{0}
$$

where $\boldsymbol{P}_{\boldsymbol{x}_{\mathbf{0}}}$ and $P_{T}$ are the predictor directions for $\boldsymbol{x}_{\mathbf{0}}$ and $T$, respectively. The predictor vector is simply the null space of the matrix on the left of Eq. 4.6. Once calculated, the predictor vector is normalized to one, as only the direction is needed. The initial state vector and frequency is then advanced by

$$
\left[\begin{array}{c}
\boldsymbol{x}_{\mathbf{0} \boldsymbol{p}^{(j)}} \\
T_{p}^{(j)}
\end{array}\right]=\left[\begin{array}{c}
\boldsymbol{x}_{\mathbf{0}}{ }^{(j)} \\
T^{(j)}
\end{array}\right]+s^{(j)}\left[\begin{array}{c}
\boldsymbol{P}_{x_{0}}^{(j)} \\
P_{T}^{(j)}
\end{array}\right]
$$

with $s$ representing the step size used in the predictor step and $j$ being the current corrector step. The step size magnitude and sign are unfixed and are calculated by

$$
s^{(j)}=\operatorname{sign}\left(s^{(j-1)} \mathbf{p}^{(j)^{T}} \mathbf{p}^{(j-1)}\right)\left(\frac{K^{*}}{K^{(j-1)}}\right)
$$

where $K^{(j-1)}$ is the number of Newton-Raphson iterations that were needed to obtain the previous periodic solution and $K^{*}$ is a predefined desired number of iterations to obtain any periodic solution. The $\frac{K^{*}}{K^{(j-1)}}$ ratio acts as a governor on the arc length continuation algorithm, effectively slowing it down in regions of high sensitivity to system parameters. Also, it should be noted that an initial step size is needed and is determined via trial and error method. After the predictor step is calculated, the corrector step is implemented in an attempt to refine the predicted variables to a periodic solution. This process is similar to the shooting method, except the direction of the Newton-Raphson iteration steps are restricted to be orthogonal to the tangent predictor vector to increase convergence rate. The set of equations describing the corrector step is given as: 


$$
\left[\begin{array}{ll}
\frac{\partial H}{\partial \boldsymbol{x}_{\mathbf{0}}} & \frac{\partial H}{\partial T} \\
\boldsymbol{P}_{\boldsymbol{x}_{\mathbf{0}}} & P_{T}
\end{array}\right]\left[\begin{array}{c}
\Delta \boldsymbol{x}_{\mathbf{0}} \\
\Delta T
\end{array}\right]=\left[\begin{array}{c}
-H\left(\boldsymbol{x}_{\mathbf{0}}, T\right) \\
0
\end{array}\right]
$$

The Newton-Raphson based shooting method is implemented on this system until the shooting function is converged. In the event that the corrector step diverges or converges slowly, the predictor step can be reimplemented but with the step size divided by two. After successful corrector step convergence, the amplitude of the periodic solution is recorded and is used as a single point on the frequency response curve. After the frequency range of interested is covered, the finished response curve is plotted. An illustrative example is given in Fig. 4.6.

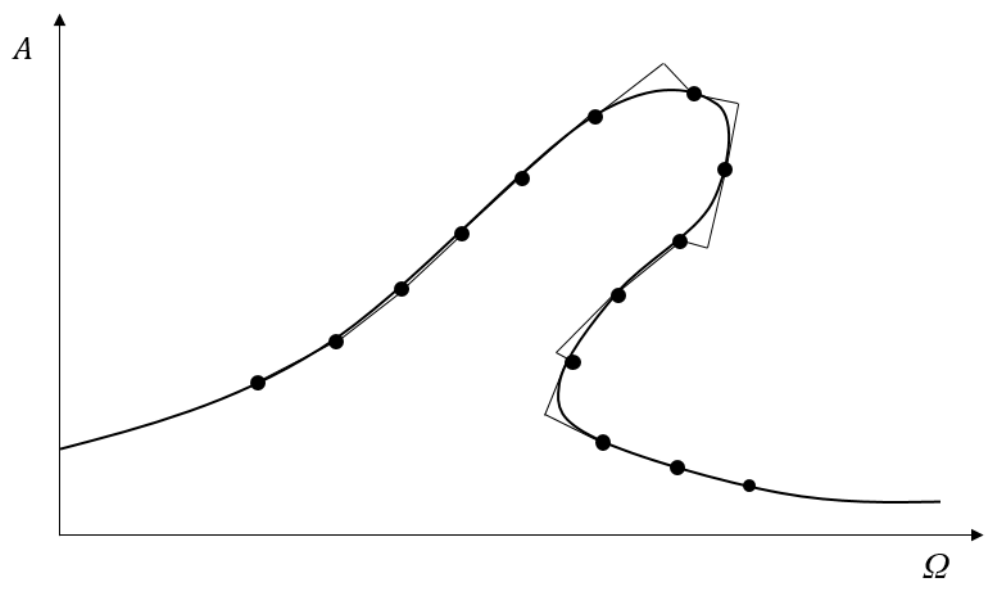

Figure 4.6: Arc-Length Continuation for Nonlinear System

\subsubsection{Stability of Periodic Orbits}

Once a periodic orbit has been established, it is important to classify the stability of such solution. In general terms, stability analysis aims to determine the sensitivity of a periodic solution to perturbations of its initial conditions. Consider the dynamic system in state space representation 


$$
\dot{x}=f(x)
$$

where $x\left(t, \boldsymbol{x}_{\mathbf{0}}\right)$ is the state vector dependent on time $t$ is time and initial state vector $\boldsymbol{x}_{\mathbf{0}}$. Differentiating Eq. 4.10 with respect to $\boldsymbol{x}_{\mathbf{0}}$ gives

$$
\frac{\partial}{\partial \boldsymbol{x}_{\mathbf{0}}}\left(\dot{x}\left(t, \boldsymbol{x}_{\mathbf{0}}\right)\right)=\frac{\partial f\left(x\left(t, \boldsymbol{x}_{\mathbf{0}}\right)\right)}{\partial \boldsymbol{x}_{\mathbf{0}}}
$$

Implementing the chain rule on the right hand side of Eq. 4.11

$$
\frac{d}{d t}\left(\frac{\partial x\left(t, \boldsymbol{x}_{\mathbf{0}}\right)}{\partial \boldsymbol{x}_{\mathbf{0}}}\right)=\left(\frac{\partial f\left(x\left(t, \boldsymbol{x}_{\mathbf{0}}\right)\right)}{\partial \boldsymbol{x}_{\mathbf{0}}}\right)\left(\frac{\partial x\left(t, \boldsymbol{x}_{\mathbf{0}}\right)}{\partial \boldsymbol{x}_{\mathbf{0}}}\right)
$$

Renaming $\frac{\partial x\left(t, \boldsymbol{x}_{0}\right)}{\partial \boldsymbol{x}_{\mathbf{0}}}$ as $\boldsymbol{\Phi}$, and $\frac{\partial f\left(x\left(t, \boldsymbol{x}_{0}\right)\right)}{\partial \boldsymbol{x}_{0}}$ as $\boldsymbol{A}$, Eq. 4.12 takes the form of

$$
\dot{\Phi}\left(t, x_{0}\right)=A \Phi\left(t, x_{0}\right)
$$

The fundamental solution matrix, $\boldsymbol{\Phi}$, is the solution of an initial value problem given in Eq. 4.13 with initial condition vector

$$
\boldsymbol{\Phi}\left(t_{0}, \boldsymbol{x}\left(\boldsymbol{t}_{\mathbf{0}}\right)\right)=\boldsymbol{I}
$$

where $\boldsymbol{I}$ is an identity matrix. The eigenvalues of the fundamental solution matrix are called Floquet Multipliers. If at least one of the Floquet Mulitpliers lies outside of a unit circle on the complex plane, then the solution is deemed unstable as shown by Fig. 4.8. Otherwise, the solution is stable as shown by Fig. 4.7.

Numerically, the fundamental solution matrix can be found by finite-difference approximation after perturbing each initial condition successively and numerically integrating the system.

In this work, the arc-length continuation method will be used to analyze the response of the beam models to varying system parameters. Stability classification 


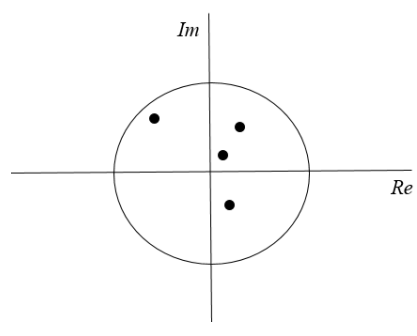

Figure 4.7: Stable Orbit

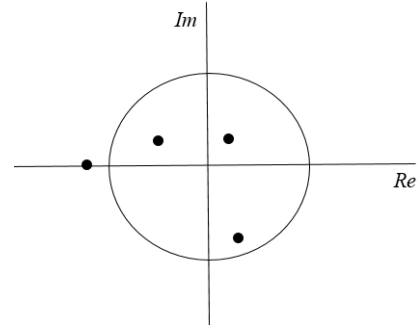

Figure 4.8: Unstable Orbit

will be performed as intermediate calculations in the continuation method, to identify the stability of each periodic solution. 


\section{CHAPTER V}

\section{Optimization Approach}

In this chapter, an approach for optimization of nonlinear dynamical systems is presented. We aim to define an optimization problem and constraints that, once solved, ensures system stability, periodicity, and amplified response in a manner that is more efficient, robust, and capable than continuation.

\subsection{Motivation}

The arc-length continuation method presented in Chapter IV is an appropriate and useful tool for determining the response amplitude as a function of a system parameter. When additional system parameters are varied in conjunction with the forcing frequency, the continuation method becomes less effective. As stated by Liao (2013), "[...] the combined effects of parameter variations on the peak vibration amplitude cannot be investigated via the continuation method when all of the parameters studied vary simultaneously." The reason for this is that each periodic response calculated in the continuation method depends entirely on the previous response parameters. Predicting the necessary parameters that produce the peak amplitude requires significant design space bracketing and knowledge of parameters values close to the peak. Even with this knowledge, the arc-length continuation method simply cannot vary the initial condition vector, forcing frequency and additional system parameters 
simultaneously. A possible solution might involve performing a full arc-length continuation "sweep" for every parameter variation and recording the peak amplitude as an objective function evaluation at that parameter value. While potentially feasible, this approach becomes increasingly inefficient with more systems parameters.. The requirement of Floquet stability of the solution also creates more complications as this effectively decreases the size of a feasible design region. Because of this ineffectiveness of the continuation method to produce maximized, stable, periodic responses with multiple system parameters, a new optimization method and approach is needed.

\subsection{Problem Formulation}

The proposed approach combines classical optimization procedures with the nonlinear analysis tools and techniques presented earlier in this work. Instead of full design space exploration via continuation, we will recast various stages of continuation as constraints on an optimization problem. Given a dynamic system with response, $x(t)$, we aim to maximize its amplitude:

$$
\text { maximize: } f\left(\boldsymbol{x}_{\mathbf{0}}, \Omega, \boldsymbol{v}\right)=\frac{\left(x_{\max }(t)-x_{\min }(t)\right)}{2}
$$

The objective function of Eq. 5.1 is a function of the initial state vector, $\boldsymbol{x}_{\mathbf{0}}$, the forcing frequency, $\Omega$, and a vector of system parameters, $\boldsymbol{v}$. To ensure periodicity of the maximized solution, we define a nonlinear inequality constraint based on the shooting function:

$$
H\left(\boldsymbol{x}_{\mathbf{0}}, \Omega, \boldsymbol{v}\right)=\max \left|\boldsymbol{x}_{\boldsymbol{T}}-\boldsymbol{x}_{\mathbf{0}}\right|<\epsilon
$$

where $\epsilon$ is a maximum allowable error for convergence. In addition to periodicity, it is desired that the maximized solution is also dynamically stable. To ensure this, we define an additional nonlinear inequality constraint that is based on Floquet stability: 


$$
g\left(\boldsymbol{x}_{\mathbf{0}}, \Omega, \boldsymbol{v}\right)=\max |\boldsymbol{\rho}|<1
$$

where $\boldsymbol{\rho}$ is a vector of Floquet multipliers for a given system response. The optimization problem is thus presented completely:

$$
\begin{array}{r}
\text { maximize: } f\left(\boldsymbol{x}_{\mathbf{0}}, \Omega, \boldsymbol{v}\right)=\frac{\left(x_{\max }(t)-x_{\min }(t)\right)}{2} \\
\text { s.t. }\left\{\begin{array}{l}
H\left(\boldsymbol{x}_{\mathbf{0}}, \Omega, \boldsymbol{v}\right)=\max \left|\boldsymbol{x}_{\boldsymbol{T}}-\boldsymbol{x}_{\mathbf{0}}\right|<\epsilon \\
g\left(\boldsymbol{x}_{\mathbf{0}}, \Omega, \boldsymbol{v}\right)=\max |\boldsymbol{\rho}|<1
\end{array}\right.
\end{array}
$$

Additional constraints can be imposed, as needed, to further govern a desired response. Examples of such constraints include limiting displacement, velocity, or mechanical stress. Eq. 7.1 along with the nonlinear constraints in Eq. 5.5 can be solved with traditional optimization methods without the need for intermediate shooting method applications to ensure periodicity. The solution will be maximized, periodic, and stable. This approach is, in essence, a multiobjective optimization problem in which the objectives have been recast as nonlinear constraints. This adjustment allows the chosen optimization algorithm to focus on a single objective function subject to constraints instead of mitigating multiple objectives via linear scaling or tradeoff solution evaluation.

We must now select an appropriate computational algorithm to perform the optimization iterations.

\subsection{Optimization Algorithm Selection}

While traditional optimization algorithms can be used to solve Eq. 7.1, significant knowledge of the design space is needed. As shown in Chapter IV, there often exist multiple periodic solutions for given excitation frequencies. This issue is exacerbated 
when additional degrees of freedom are given to the problem, such as system parameter variation. Because of this issue, there is a need for an optimization algorithm that can best handle many independent variables and many local solutions.

Genetic algorithms (GA's) are suitable for problems with large, noisy design spaces with many local solutions and discontinuous functions (Ghanea-Hercock (2013)). GA's work by essentially imitating biological system genetics by encoding, "mating", and "mutating" different design variables over several generations of populations. Intermittently, the populations are sorted by fitness and the least fit solutions are discarded from the population. Repeating this process and occasionally "mutating" population members, the most fit solution should dominate as the optimization problem solution. One drawback to using GA's is the finesse required to appropriately apply the algorithm to a given problem. While this is true to some extent for all optimization algorithms, it is especially crucial for GA's . The choices for encoding procedure, population size, mating frequency, mutating frequency, and other metrics often require a lot of trial and error. Furthermore, GA's are good at finding regions of interest within large design spaces, but often struggle with the fine tuning toward the optimal solution. The reason for this struggle, is that the precision of the algorithm depends almost entirely on the encoding procedure. A more refined encoding process can help alleviate local convergence issues, but at the expense of computation time (Melanie (1999)).

Another optimization algorithm of potential interest is that of surrogate modeling. Surrogate modeling optimization aims to iteratively approximate the shape of the design space by means of numerical models. In general, surrogate modeling casts a set of trial points into the design space, evaluates their objective function values, and then performs regression curve fitting to approximate the space. A local optimization solver is then employed to find the minimum of the surrogate. As more trials points are added to the space, the surrogate model can become more representative of the 
true design space. The issue arises, however, of choosing an appropriate surrogate model. A priori heuristics about the space is typically needed to choose the surrogate that best describes the true design space. Furthermore, for spaces with multiple minima, a surrogate model can iterate toward an undesired minima, or can completely misrepresent the space (Mack et al. (2007)).

The last potential optimization algorithm for our problem is a multi-start approach in which local optimization solvers are employed at multiple points in the design space. Essentially, a set of trial points is cast into the design space, and local gradientbased algorithms work independently to find local minima. After convergence (or divergence) of all trial points is reached, the local solutions that were found are compared and the best set are kept. Additional trial points are then recast at locations near the areas of most feasible solutions and the gradient searches are repeated. Upon completion of the algorithm, the method returns the best solution found of all local searches. The algorithm is powerful in that it can search throughout large design spaces with multi minima. The local solver can be tailored to the problem at hand, to best mitigate any potential local convergence issues. The multi-start algorithm requires selection of the number of trial points as well as selection of the local solver algorithm. The algorithm also typically requires relatively smooth design spaces and continuous objective functions as the local solvers typically make use of the local gradient (Ugray et al. (2007)).

With the optimization algorithm options considered, a multi start type algorithm was selected for our problem. The method has a high potential for success because of three main reasons. First, with multiple minima and relatively large design spaces, our problems requires a large design space survey to find feasible regions for exploration. A multi start algorithm is able to cast many exploratory trial points throughout the space and find multiple local solutions. Second, high tolerances on local convergence is needed as the objective function and constraints are very sensitive to the design 
variables. Using a gradient-based local solver, a multi start algorithm can converge to near absolute minima at a local solution. Lastly, it might be wise to use a gradientbased algorithm as the shooting function constraint has shown excellent convergence with gradient methods in the past (via arc-length continuation) (Liao (2013)). A multi start method can employ many types of gradient local solvers.

\subsubsection{Scatter Search Optimization Algorithm (SSOA)}

The optimization method to be used in this study is the Scatter Search algorithm designed and developed by Egea et al. (2014). The algorithm performs multiple local optimization searches to converge to multiple feasible solutions. These local optimization operations are scattered throughout the entire design space by means of a Latin Hypercube sampling methodology. This approach maximizes the distance between starting points for local exploration and create a diverse design space exploration set. The local solution that gives rise to the best objective function value as well as satisfies all constraints is taken as the global solution.

The first step in the Scatter Search algorithm is to generate a set of initial trial points. Those points are then evaluated for constraint violation as well as objective function value. For demonstration, Fig. 5.1 shows a two-parameter design space with multiple local minima, represented by square markers, and nine initial trial points, represented by circle markers.

After each point in the diverse set of trials is evaluated, a reference set is then created based on several criteria: trial point objective function value (fitness), distance from other trial points (diversity), and constraint violation (feasibility). The reference set is typically much smaller than the diverse set. Figure 5.2 shows the selection of the reference set. The original trial points that are not included in this set are discarded permanently.

The optimization algorithm then employs a gradient-based local algorithm to 


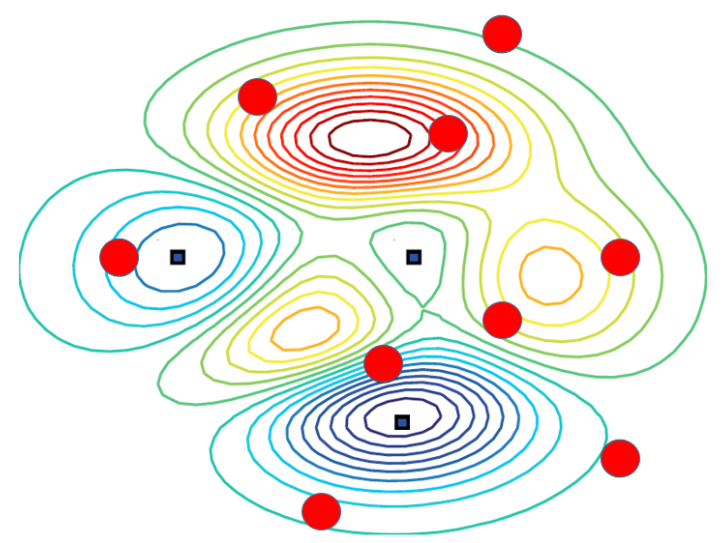

Figure 5.1: Diverse Set of Trial Points

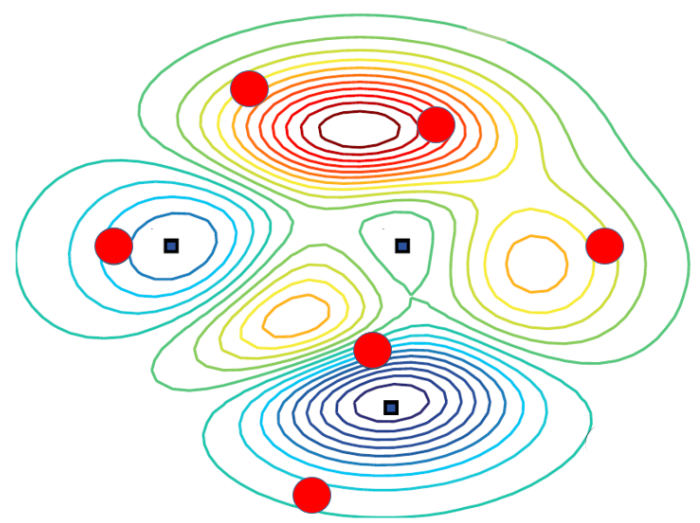

Figure 5.2: Reference Set

perform local design space exploration. Each local search terminates after either a maximum number of function evaluations is attained or a minimum change in objective function is reached (indicating a local solution). This local search operation is illustrated in Fig. 5.3.

After completion of the local searches, the Scatter Search algorithm recasts a new set of trial points based upon Latin Hypercube Sampling, as well as information gained from the local searches (constraint violations within design space, and objective function values). A new reference set is then created and local searches are performed again. This process continues until a pre-specified number of local searches is completed or a maximum computational evaluation time is reached. The algorithm records the objective function value reached at each local minimum as 


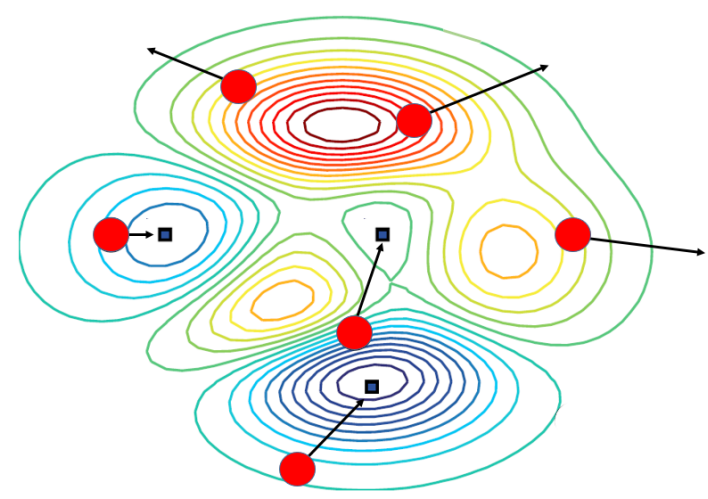

Figure 5.3: Local Gradient Optimization

well as the constraint violations. A vector of design variable values along with the associated objective function value is returned at the completion of the optimization.

At this point, we present a review of our approach to optimizing the nonlinear beam models. Figure 5.4 shows a summary of our method.

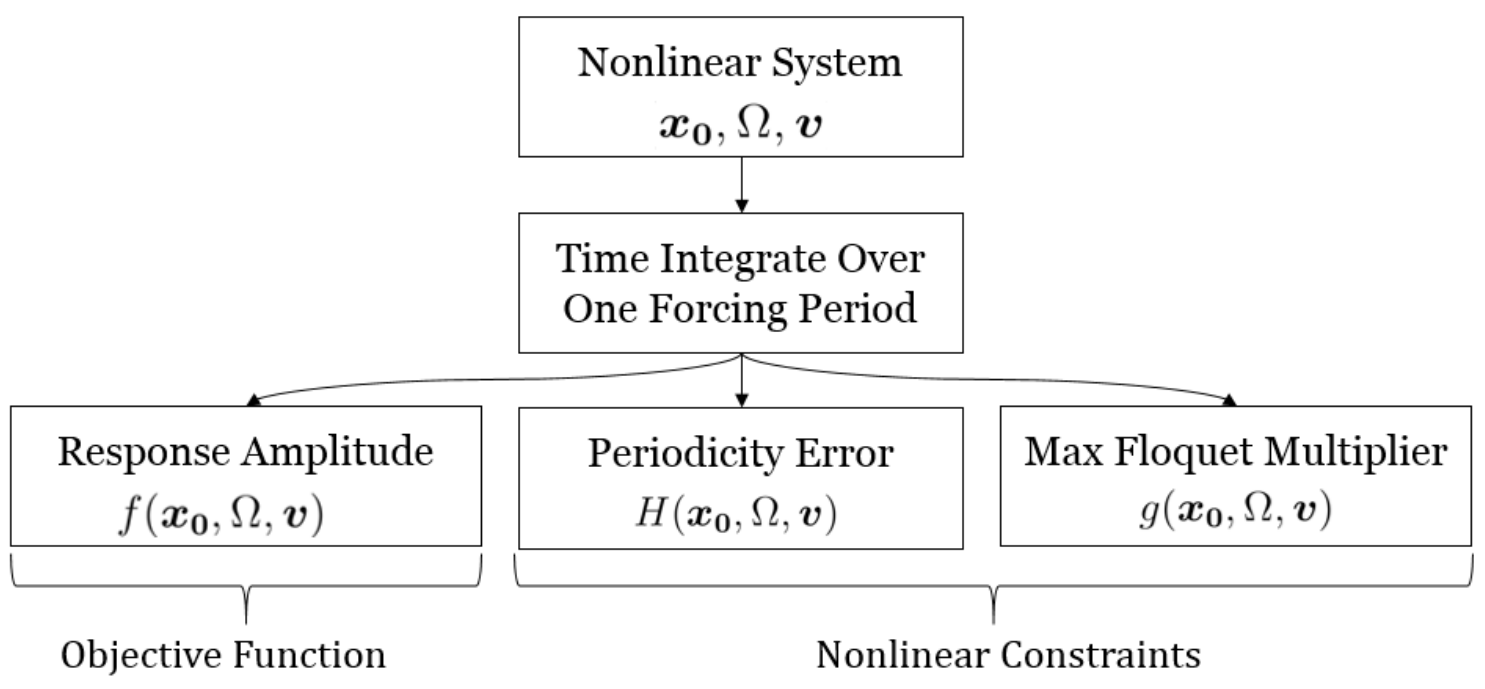

Figure 5.4: Optimization Approach Overview

In short, we are recasting the shooting method and Floquet stability criteria as nonlinear constraints in a global optimization problem. The SSOA will be employed to maximize response amplitudes of the nonlinear beam models, while ensuring both periodicity and stability of the solutions. 


\section{CHAPTER VI}

\section{Model Validation and Parametric Studies}

In this chapter, the beam models derived in Chapter III will be validated using experimental work provided by Tseng and Dugundji (1970). Parametric studies of the beam models will be presented to illustrate potential areas for nonlinear dynamic exploitation.

\subsection{Continuation Method Verification}

The beam dimensions and properties used in this research are taken from Tseng and Dugundji (1970) and are given in Table 6.1. The linear Euler-Bernoulli beam model previously presented will act as a starting point for analysis. This model will be used to verify the arc-length continuation method's ability to calculate and evolve periodic orbits. After this is verified, the continuation method will then be used to explore the dynamic behavior of the other beam models.

Recall the previously derived Euler-Bernoulli beam model:

$$
\rho A \ddot{q_{m}}+E I k_{m}^{2} q_{m}=A_{m} f(\zeta, t)
$$

with boundary conditions previously stated. With a velocity-dependent damping term, Eq. 6.1 becomes 
Table 6.1: Nominal Beam Dimensions and Material Properties

\begin{tabular}{lll}
\hline Dimension & Value & Unit \\
\hline Length, $\boldsymbol{L}$ & 18.0 & $\mathrm{in}$. \\
Width, $\boldsymbol{c}$ & 0.5 & $\mathrm{in}$. \\
Thickness, $\boldsymbol{h}$ & 0.021 & $\mathrm{in}$. \\
Elastic Modulus, $\boldsymbol{E}$ & $29 \times 10^{6}$ & $\mathrm{psi}$. \\
Density, $\boldsymbol{\rho}$ & 0.28 & $\mathrm{lb} / \mathrm{in}^{3}$ \\
Midspan Rise, $\boldsymbol{b}$ & 0.0315 & $\mathrm{in}$. \\
Base Excitation Amplitude, $\boldsymbol{W}_{\boldsymbol{B}}$ & 0.2 & $\mathrm{in}$. \\
\hline
\end{tabular}

$$
\rho A \ddot{q_{m}}+E I k_{m}^{2} q_{m}+c \dot{q_{m}}=A_{m} F \sin (\Omega t)
$$

where $c=2 \zeta_{d} \rho A \omega_{n}$ and $\zeta_{d}$ is the damping coefficient found experimentally to be 0.001 via logarithmic decrement. For this validation model, we will use an external force amplitude of $0.2 \mathrm{lbs}$. To test the arc-length continuation method, Eq. 6.2 is integrated with arbitrary initial conditions and for an extended amount of time until steady state behavior is observed. The integration uses MATLAB's built-in integrator, ODE45, which employs a variable step Runge-Kutta method to solve differential equations. By manual inspection, initial conditions are selected from the periodic, steady-state, response and are used as the first step in the arc-length continuation method. With the starting values gathered from the time integration, the arc length continuation method is then implemented. The periodic responses in generalized coordinates are then converted to physical displacement measured at the midpoint of the beam. Furthermore, the response amplitude is normalized by dividing the response by the beam thickness. Figure 6.1 shows the frequency response of the straight beam with Frequency Ratio representing the forcing frequency divided by the fundamental frequency of the beam system. Also shown in Fig. 6.1 is the analytical frequency response found via lumped parameter closed form expressions (see Appendix A).

Each data point on Fig. 6.1 represents the steady state amplitude of the straight 


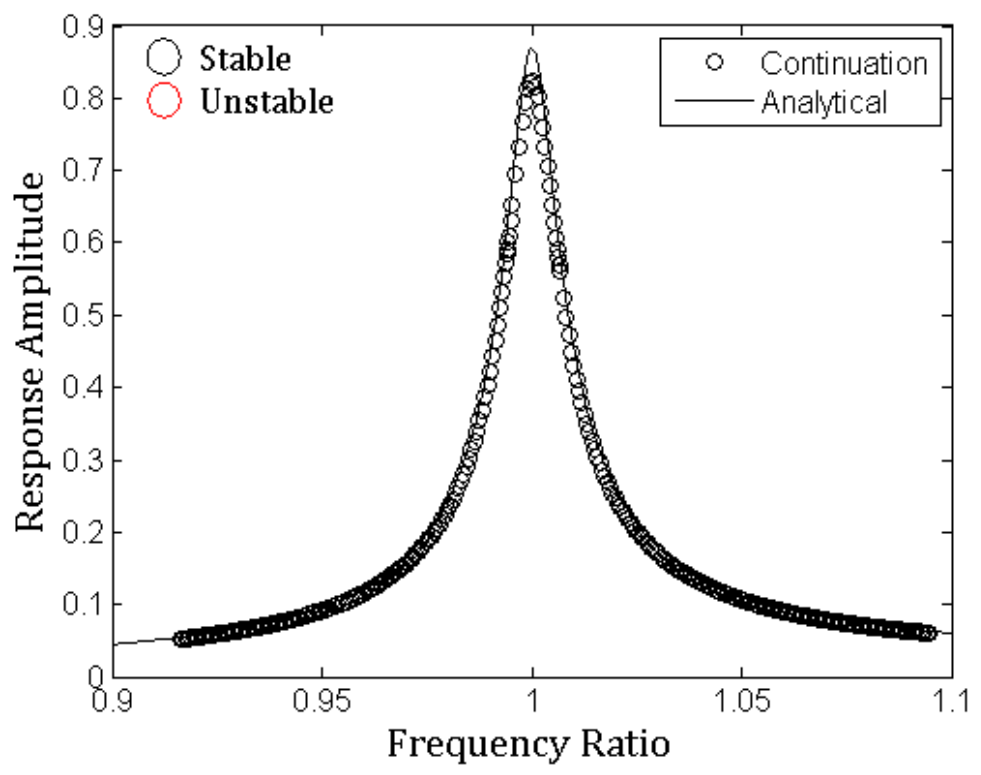

Figure 6.1: Linear Frequency Response

beam response at that forcing frequency. All of the points are black colored indicating that all periodic solutions in this frequency range of interest are dynamically stable. Furthermore for every forcing frequency there is only one possible periodic solution, which is consistent with linear systems. We also see excellent agreement between the arc-length continuation solution and the analytical solution. With the arc-length continuation method verified, we continue to the analysis of the nonlinear beam models.

\subsection{Nonlinear Straight Beam Model Response}

The large deflection straight beam model is restated

$$
\rho A \ddot{q_{m}}+E I k_{m}^{2} q_{m}+c \dot{q_{m}}+\frac{E A}{2 L} \sum_{m, n, i, j=1}^{N} A_{m n i j} q_{n} q_{i} q_{j}=B_{m} F \sin (\Omega t)
$$

with boundary conditions and constants previously defined. Tseng and Dugundji (1970) experimentally excited this beam configuration (Table 6.1) by means of a 
shaker table. The shaker table's excitation amplitude was $0.2 \mathrm{in}$. In order to convert this excitation displacement into a transverse force in our model, we differentiate the sinusoidal displacement function twice (with respect to time) and multiply by an inertia term. This results in an acceleration-inertia term, that converts the physical shaker displacement into a transverse force. This modification is given as

$$
\rho A \ddot{q_{m}}+E I k_{m}^{2} q_{m}+c \dot{q_{m}}+\frac{E A}{2 L} \sum_{m, n, i, j=1}^{N} A_{m n i j} q_{n} q_{i} q_{j}=-B_{m} \rho A W_{B} \Omega^{2} \sin (\Omega t)
$$

where $W_{B}$ is the physical excitation displacement amplitude of the shaker.

We next apply a single mode discretization and produce a frequency response plot via the arc-length continuation method as shown in Fig. 6.2.

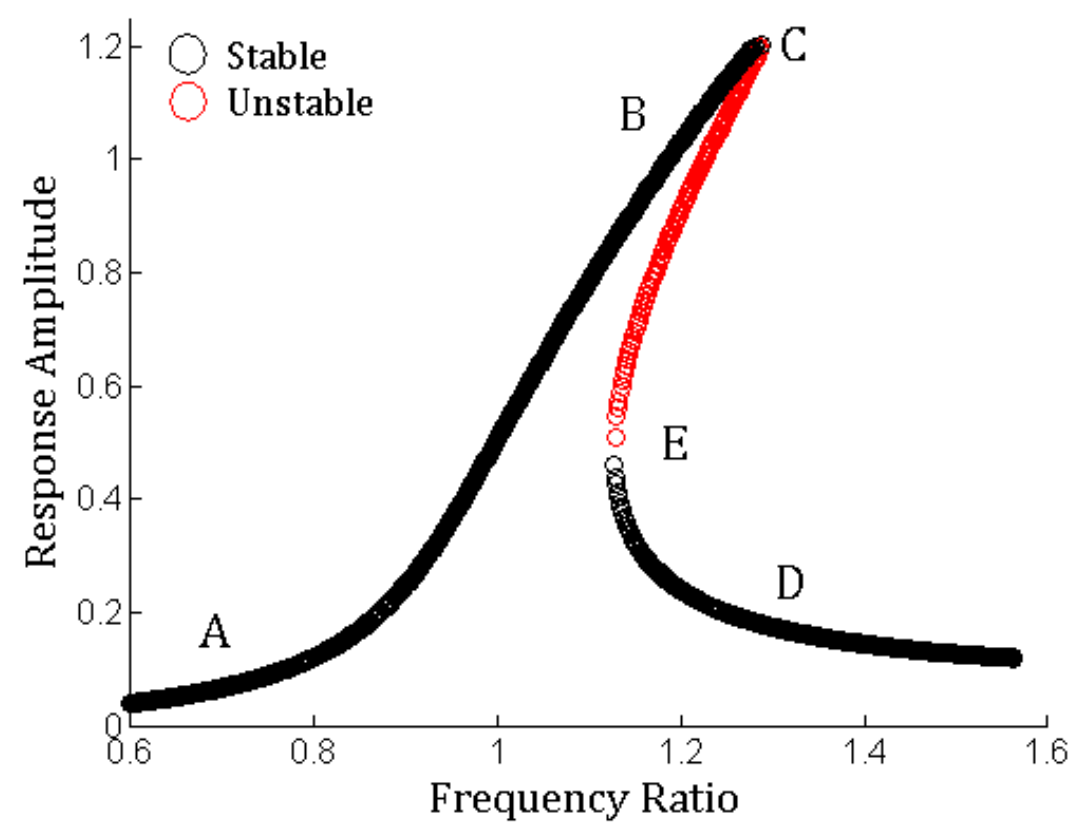

Figure 6.2: Large Deflection Beam Frequency Response (Single Mode Discretization)

There are several key nonlinear phenomena that give rise to the frequency response of Fig. 6.2. The first phenomenon is frequency shifting. This event is illustrated by the peak of the frequency response function shifting from typical linear resonance 
$($ Frequency Ratio $=1)$ to a different value (Frequency Ratio $\approx 1.3)$. When a shift occurs toward higher frequency values, a hardening nonlinear effect is experienced. The strong hardening nonlinearity can be attributed to an increase in effective stiffness as the beam is stretched during large transverse displacements. The second phenomenon occurring is amplitude jump. While the continuation algorithm turned the corner at point $\mathrm{C}$ as it swept from low to high frequencies, this is not necessarily representative of the true physical behavior of the beam. Instead, as the forcing frequency is swept from low to high (point A to $\mathrm{C}$ ), the response can jump from relatively high response amplitude (point $\mathrm{C}$ ), directly to much lower value amplitude (point D) and continues onward. Conversely, sweeping frequency from high to low (point D to E), the response can increase amplitude until it drastically jumps up to a much higher response amplitude (point B) and continue onward to point $\mathrm{A}$.

Another implication of the jump phenomena is that at certain forcing frequencies there exists one periodic solution per forcing frequency (Frequency Ratio $<1.1$ and Frequency Ratio $>1.3$ ), and at other frequencies there exists up to three. Highlighted in red are periodic solutions that are unstable while highlighted in black are stable solutions. The final phenomenon to observe in this system is the area contained by points C, D, E, B in Fig. 6.2. This area is evidence of nonconservative hysteresis in the system giving rise to energy loss.

\subsubsection{Model Validation}

Tseng and Dugundji (1970) performed shaker-table tests on this beam configuration and, for a sweep of frequencies, recorded the steady-state amplitude of the beam response. They then plotted the steady state amplitudes against the frequency ratio. Their results agreed with the analytical backbone curve they developed via the harmonic balance method. Using their experimental data backbone curve as a means of model validation, Fig. 6.3 shows a strong level of agreement between the model 
used here and the experiment.

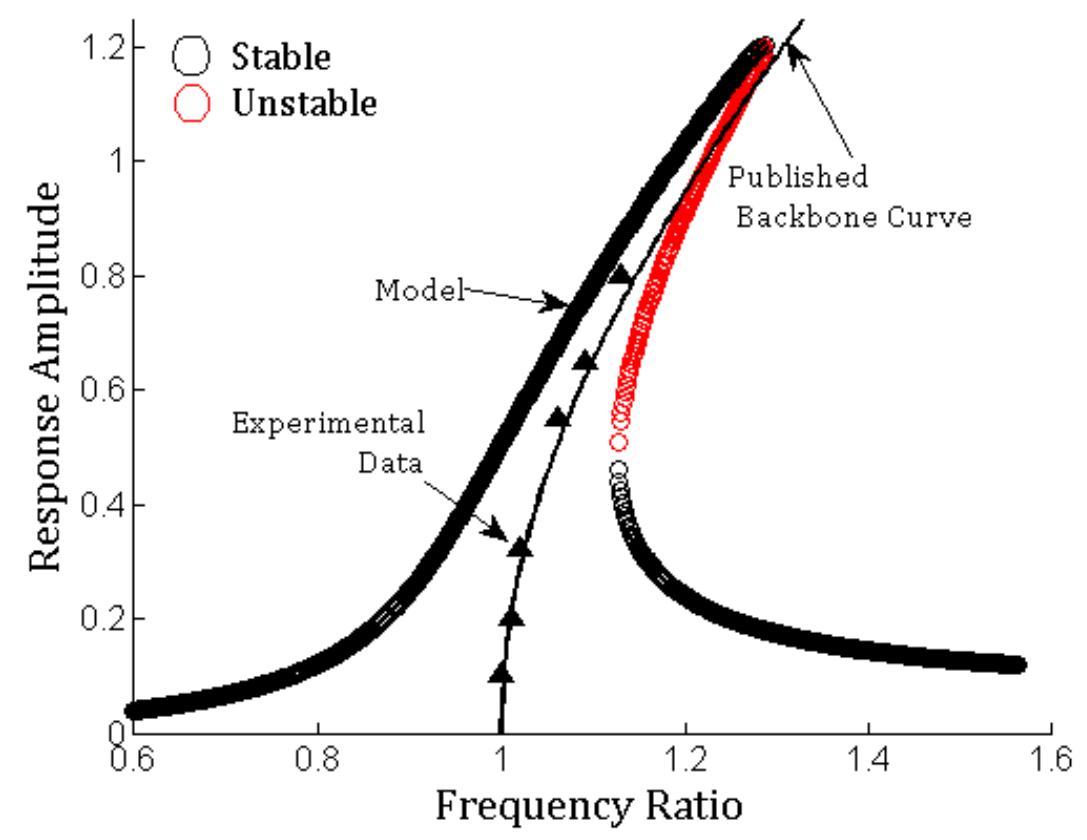

Figure 6.3: Large Deflection Beam Validation (Single Mode Discretization)

We see that our model effectively captures the nonlinear hardening behavior of the beam.

\subsubsection{Parametric Study: Beam Geometry}

Using the configuration used by Tseng and Dugundji (1970) as a control or baseline case (specifically, beam thickness, $h=0.021 \mathrm{in}$.), a parametric study with $h$ as the parameter of interest was performed. The values for $h$ that were used are: 0.0242 , $0.0231,0.0221,0.0199,0.0189$, and $0.0179 \mathrm{in}$. The results of this study are shown in Fig. 6.4. 


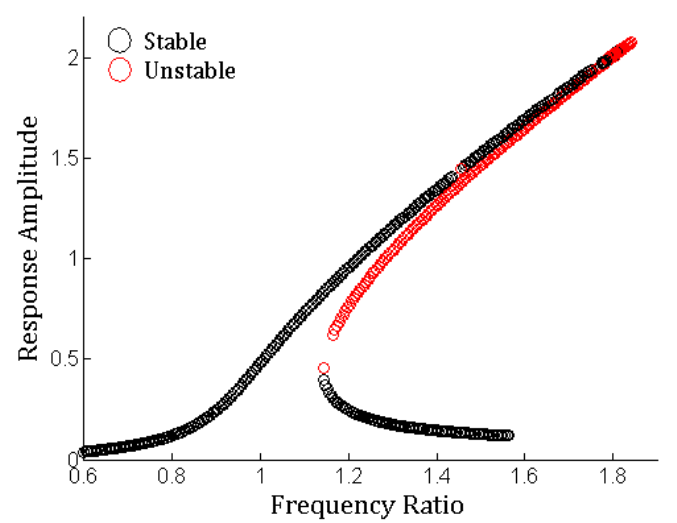

$\mathrm{h}=0.0242 \mathrm{in}$.

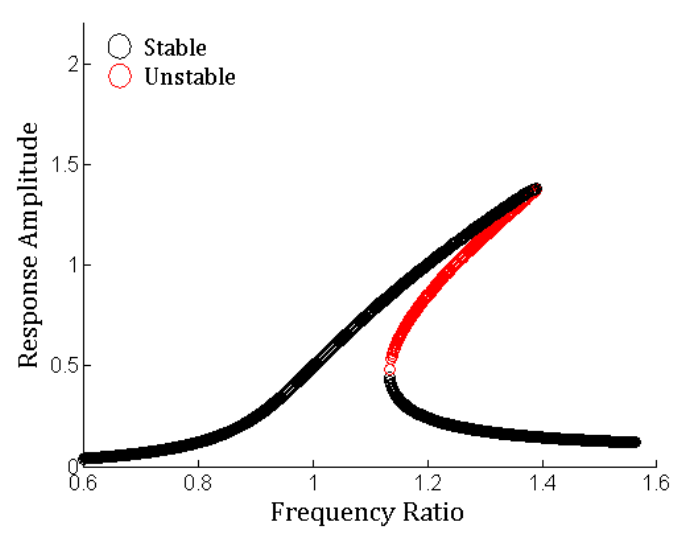

$\mathrm{h}=0.0221 \mathrm{in}$.

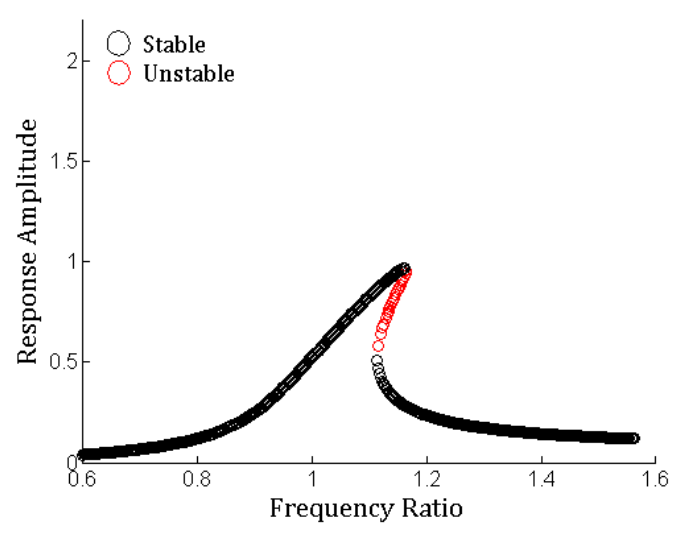

$\mathrm{h}=0.0189 \mathrm{in}$.

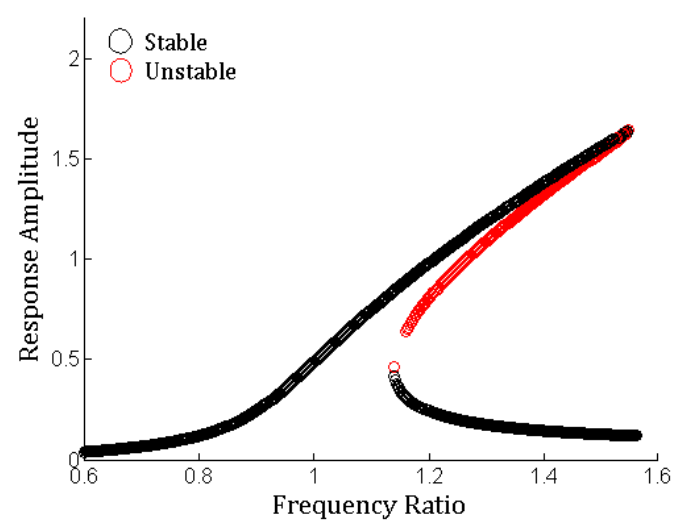

$\mathrm{h}=0.0231 \mathrm{in}$.

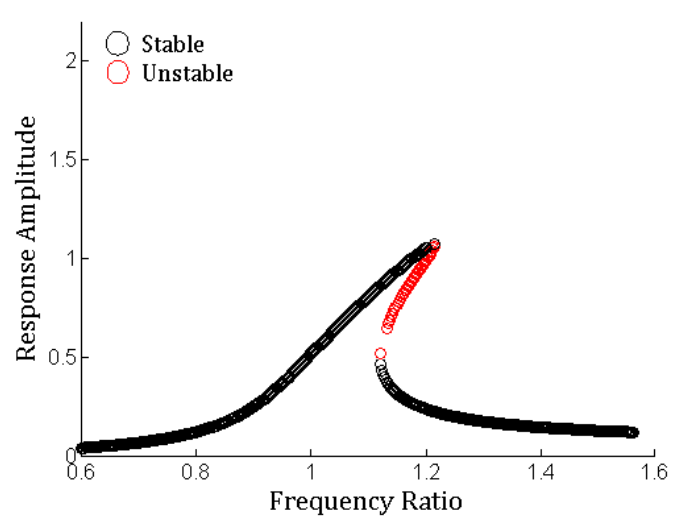

$\mathrm{h}=0.0199 \mathrm{in}$.

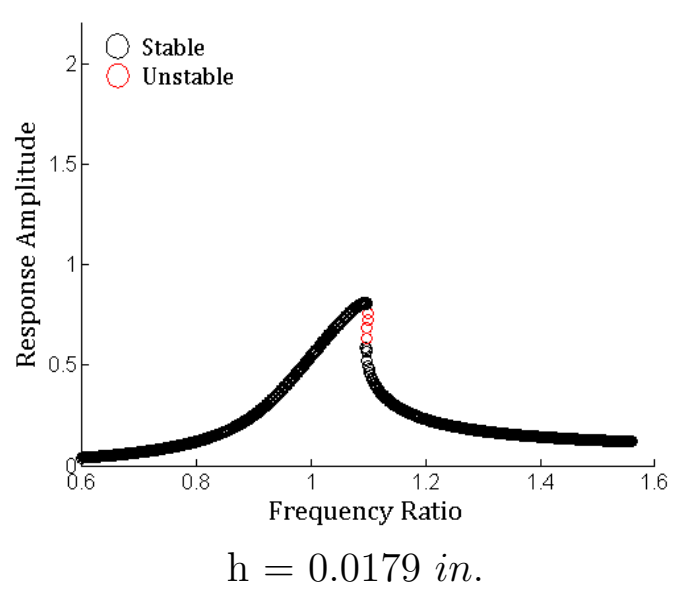

Figure 6.4: Parametric Study: Effect of Beam Thickness 
As the beam thickness increases, the effective hardening nonlinearity increase. This is due to the beam thickness increasing the beam's cross-sectional area, $A$, in Eq. 6.4, which in turn increases the coefficient of the cubic nonlinear term. The more interesting trend that can be seen is that of response amplitude amplification as the beam thickness is increased. While increasing beam thickness causes larger areas of instability, the amplitude increase shows a potential area for exploiting these nonlinear dynamics for benefit. To further explain what is occurring in this parametric study, one can view the beam thickness as an effective third axis in the dynamic space and each of the plots in Fig. 6.4 is a slice along that axis. Fig. 6.5 shows a representation of this concept.

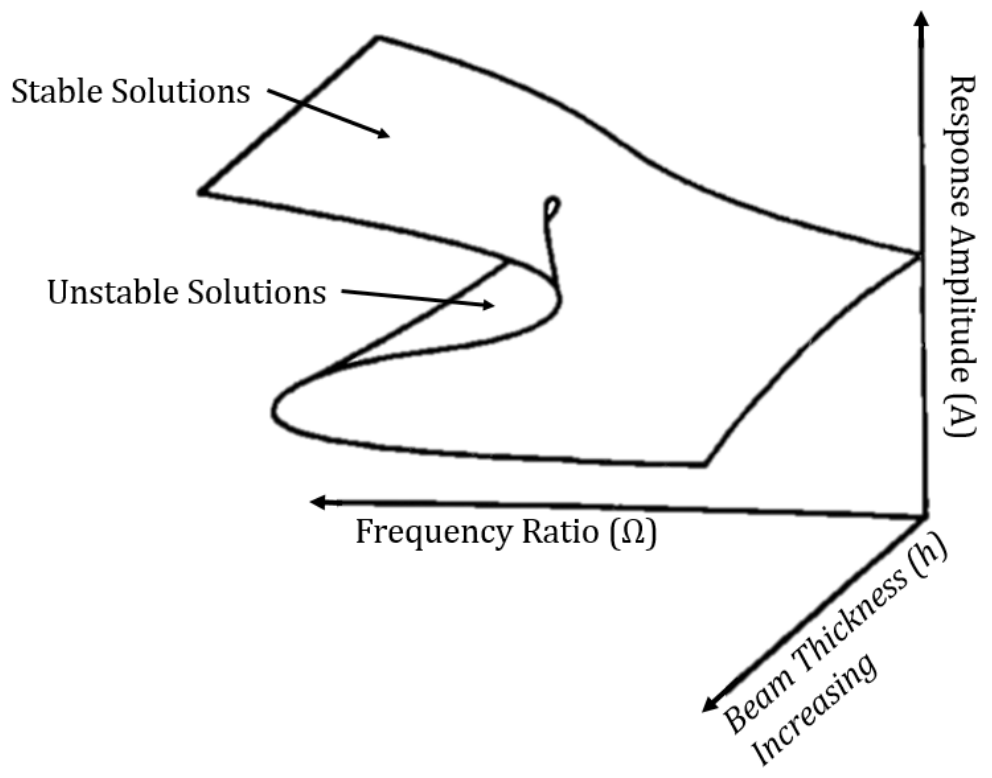

Figure 6.5: Beam Response Surface In $h-\Omega-A$ Space

Figure 6.5 depicts a cusp catastrophe, in which the underside of the manifold in the response surface represents unstable solutions. The visualization of this surface shows the feasibility of using optimization methods to find the peak response amplitude within this design space. Figure 6.5 certainly inspires the potential for response surface optimization. 


\subsection{Buckled Beam}

For convenience, the multiple mode discretized buckled beam model is restated

$$
\begin{array}{r}
\rho A \ddot{q}_{m}+c \dot{q}_{m}+P E I q_{m}+\frac{E A}{2 L}\left[\sum_{m, n, i=1}^{N} A_{m n i} q_{n} q_{i}+\right. \\
\left.\sum_{m, n, i, j=1}^{N} B_{m n i j} q_{n} q_{i} q_{j}\right]=C_{m} F \sin (\Omega t)
\end{array}
$$

Modifying the forcing term in Eq. 6.6 to account for the displacement-based shaker excitation gives

$$
\begin{array}{r}
\rho A \ddot{q}_{m}+c \dot{q}_{m}+P E I q_{m}+\frac{E A}{2 L}\left[\sum_{m, n, i=1}^{N} A_{m n i} q_{n} q_{i}+\right. \\
\left.\sum_{m, n, i, j=1}^{N} B_{m n i j} q_{n} q_{i} q_{j}\right]=-C_{m} W_{B} \rho A \Omega^{2} \sin (\Omega t)
\end{array}
$$

Tseng and Dugundji (1970) performed shaker tests on a buckled beam with an initial static rise of the midspan of the beam equal to $0.0315 \mathrm{in}$. using a compressive axial force, $P=25.09 \mathrm{lbf}$. This is approximately $5.25 \%$ greater than the critical buckling load $P_{c}=23.84 \mathrm{lbf}$. The shaker excitation amplitude as well as the material properties and dimensions of the beam in this study are the same as the previous model in Table 6.1. A single mode discretization was performed on Eq. 6.6 and then numerically integrated until steady state response was achieved. Initial conditions where then retrieved from the time response and the arc-length continuation method was implemented. After a sweep of the frequency range of interest, the frequency response of the beam at midpoint $(\zeta=0.5)$ of the buckled beam model was generated and shown in Fig. 6.6.

Response Amplitude is the physical amplitude of the beam response divided by the 


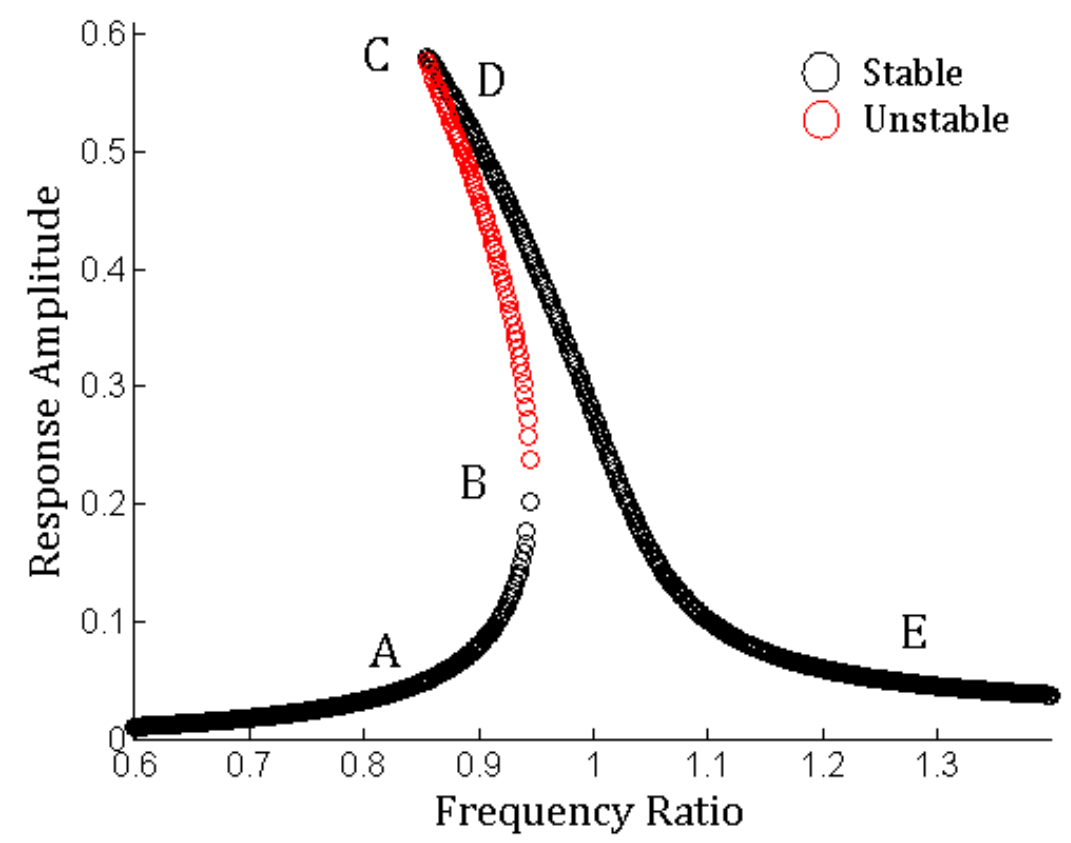

Figure 6.6: Buckled Beam Frequency Response (Single Mode Discretization)

beam thickness. The frequency response of the buckled beam model is qualitatively different from the nonlinear straight beam response. Firstly, the buckled beam model exhibits a nonlinear softening response. This is attributed to the decrease in beam stiffness at low buckling levels. This phenomenon is in agreement with Virgin and Plaut (2003) and Mallon (2008). Hysteresis and jump phenomenon are also present in the buckled beam dynamics as the response amplitude jumps from point $\mathrm{B}$ to point $\mathrm{D}$ as the excitation frequency is swept from point A toward point E. Conversely, as the excitation frequency is swept from point $\mathrm{E}$ to point A the response climbs through point $\mathrm{D}$ up to point $\mathrm{C}$ but then jumps down to a solution near point $\mathrm{B}$ and continues to point A. Points A, B, and C encompass the hysteresis response of the system giving rise to energy loss. 


\subsubsection{Model Validation}

For this configuration, Tseng and Dugundji (1970) excited the buckled beam across a range of excitation frequencies, and recorded the steady-state amplitude of the beam response. They then plotted those amplitudes against the frequency ratio. Their results agreed with the analytical backbone curve they developed via the harmonic balance method. Using this backbone curve as a means of model validation, Fig. 6.7 compares our model to the experiment.

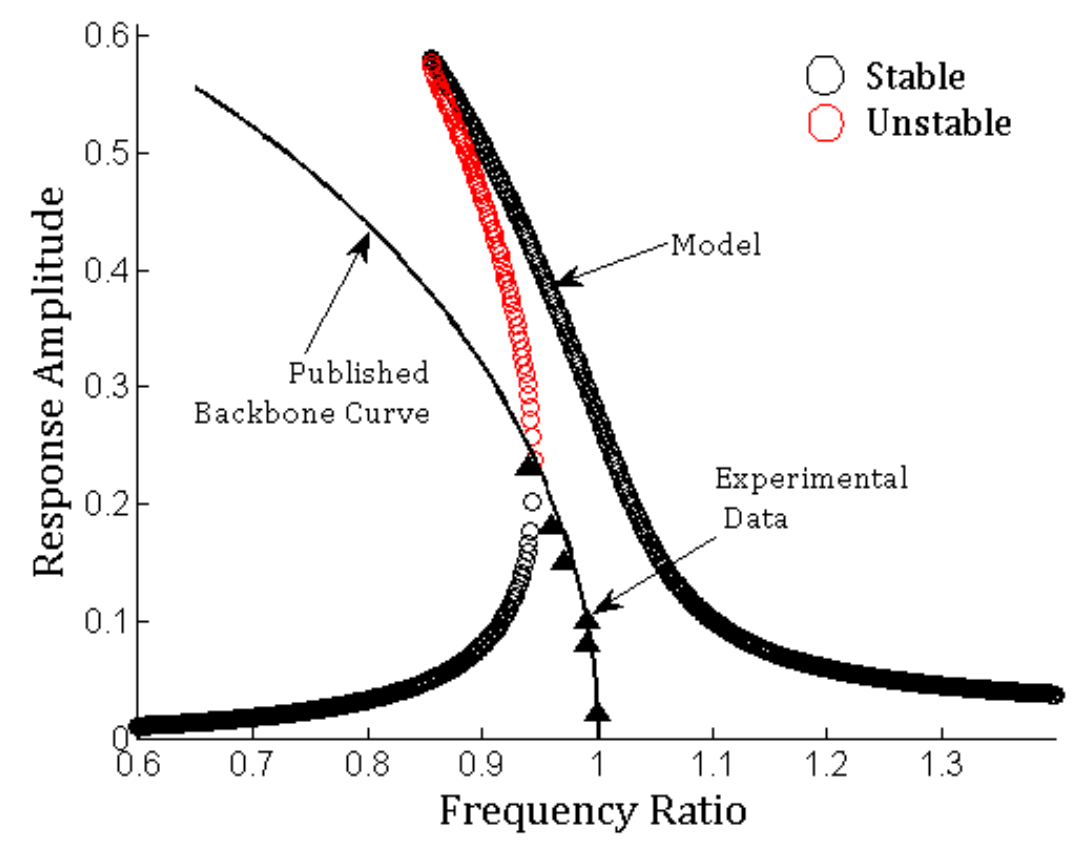

Figure 6.7: Buckled Beam Validation (Single Mode Discretization)

The buckled beam model appears to differ more significantly from the experimental data than that of the nonlinear straight beam. While a few qualitative characteristics were captured in the simulation (primarily nonlinear softening), the two responses are significantly different. Several literature sources Lacarbonara (1997), Emam (2002)), and (Mestrom (2009) have shown that the number of modes included in the discretization process can have an effect on effective nonlinearity and model accuracy of the first mode. As such, a second vibration mode was added to the 
Galerkin discretization process and the frequency response plot was generated. As the base excitation is uniform along the length of the beam, the excitation itself does not activate the second mode. Nonlinear modal interactions, however, can occur in which excitation of the first mode can activate the second mode at the first natural frequency. This phenomenon is discussed in great detail by Tseng and Dugundji (1970), Lacarbonara (1997), Emam (2002), and Yabuno et al. (2003). This coupled modal interaction of the first two modes can quantitatively and qualitatively change the response of the first mode. This change can be seen in Fig. 6.8.

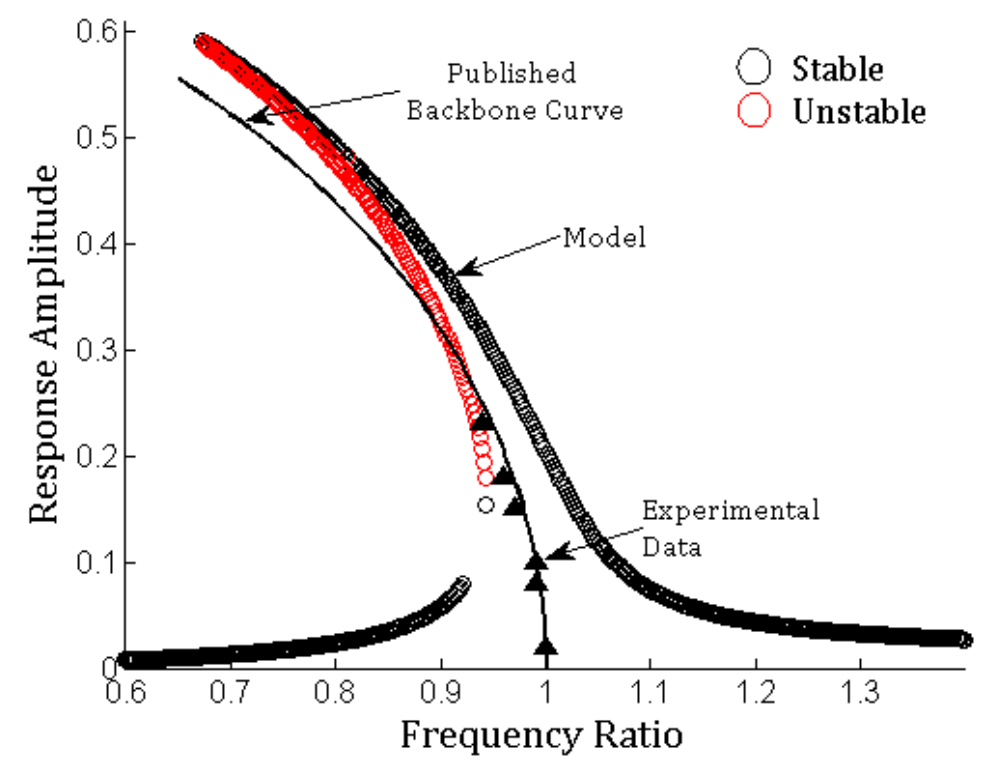

Figure 6.8: Buckled Beam Model Validation (Two Mode Discretization)

Fig. 6.8 shows better agreement between the two-mode modal and the experimental response. The model better captures the magnitude of the dynamic nonlinearly than that of the single-mode discretization. Two more state variables need to be accounted for during continuation as well as future optimization with the inclusion of each additional mode. As discussed in Chapter V, the state variables will be treated as system parameters in the optimization computations. As such, the two-mode model both captures the dynamics well and limits the degrees of freedom in the forthcoming 
optimization problem.

\subsubsection{Parametric Study: Buckling Level}

Using the configuration used by Tseng and Dugundji (1970) as a control or baseline case (specifically, midspan rise, $b=0.0315 \mathrm{in}$.), a parametric study with $b$ as the parameter of interest was performed on the two-mode model. The values for $b$ that were used are: $0.0252,0.0315,0.0347,0.0441,0.0504,0.0536 \mathrm{in}$. The results of this study are shown in Fig. 6.9. All other system parameters were held constant at nominal values. 


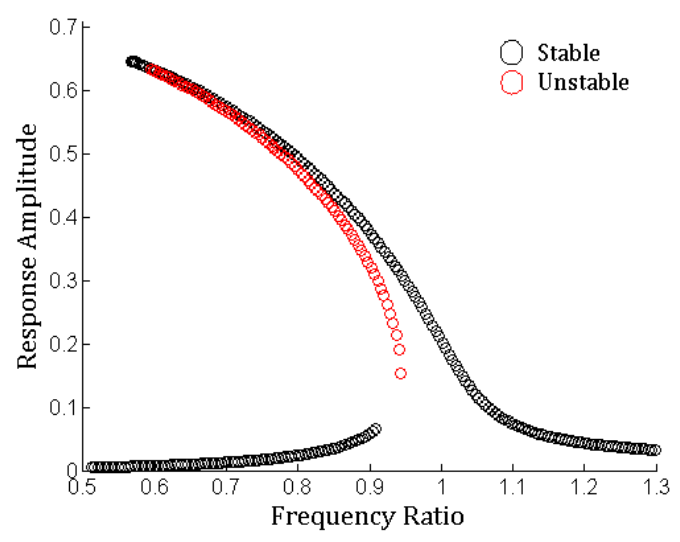

$\mathrm{b}=0.0252 \mathrm{in}$.

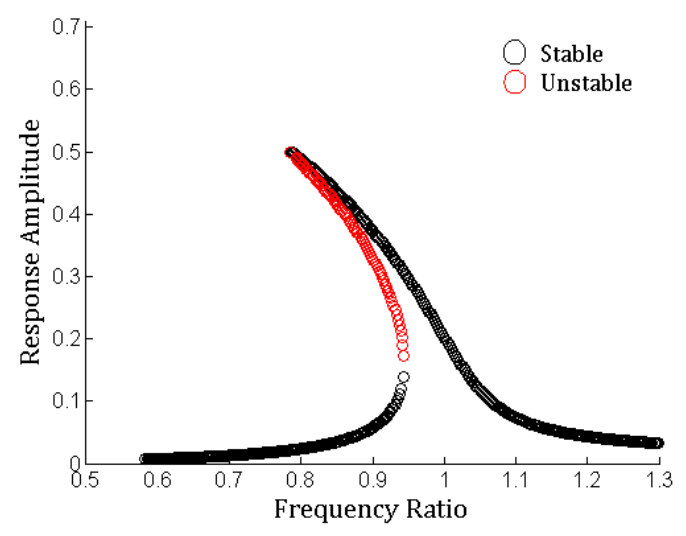

$\mathrm{b}=0.0347 \mathrm{in}$.

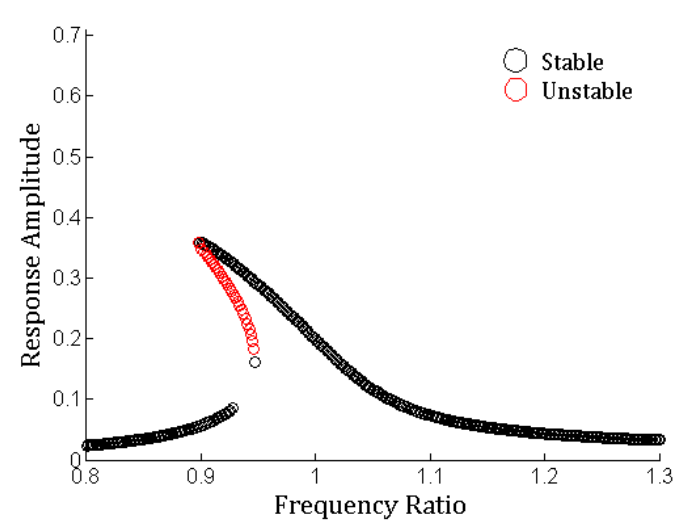

$\mathrm{b}=0.0504 \mathrm{in}$.
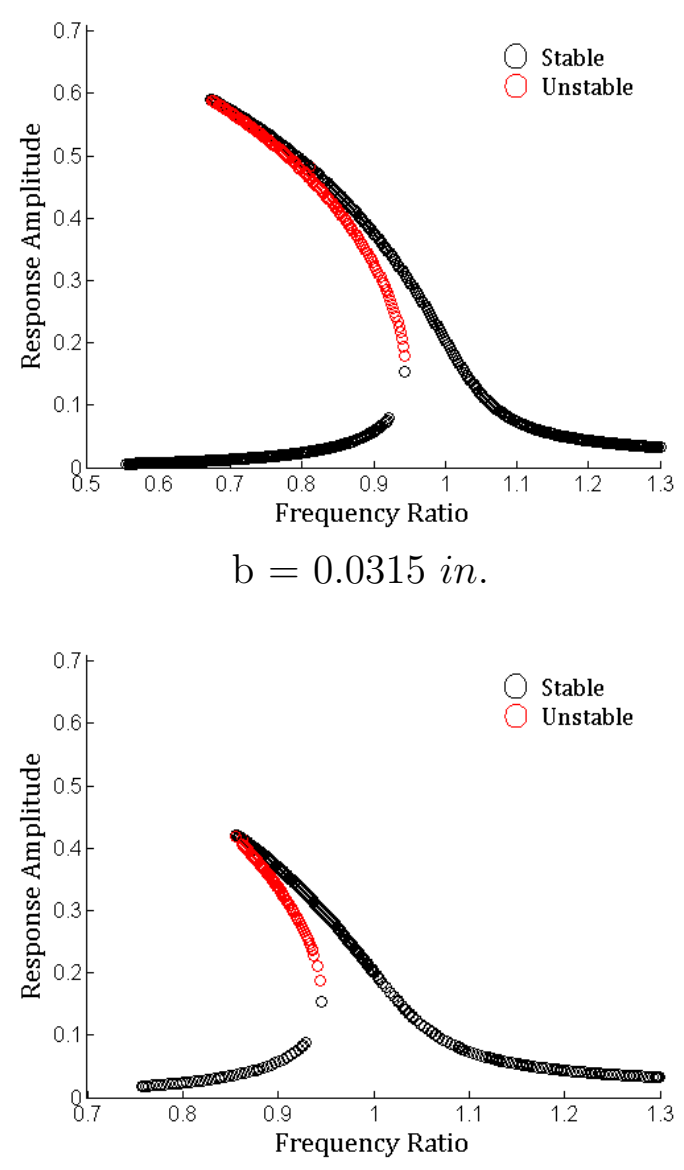

$\mathrm{b}=0.0441 \mathrm{in}$.

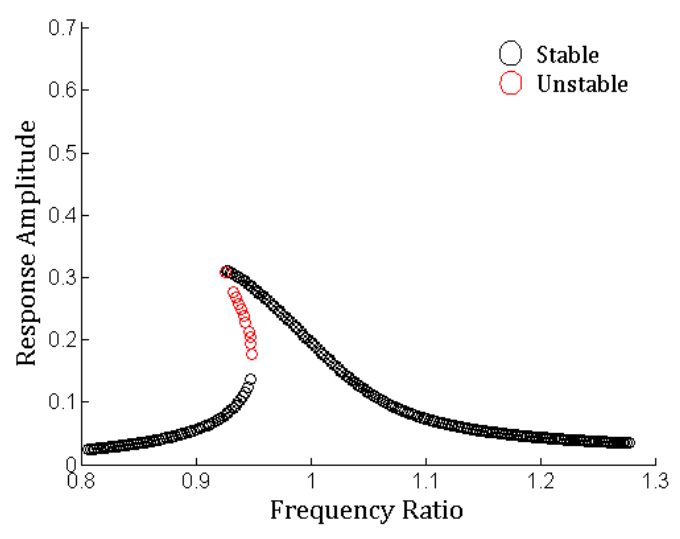

$\mathrm{b}=0.0536 \mathrm{in}$.

Figure 6.9: Parametric Study: Effect of Static Rise 
Midspan rise values closer to the buckling bifurcation point $(b=0)$, give rise to many more unstable responses near resonance. As the midspan rise is increased (as a consequence of larger axial loading), the system becomes more and stable. At $b=0.0504 \mathrm{in}$., the response becomes almost completely stable The second result of this parametric study is that with midspan rise values close to the bifurcation point, the amplitude of the peak periodic response increases. In contrast to the amplitude control as a function of a geometric quantity in the straight beam, this trend in the buckled beam is an even more promising area of exploitation. What has been effectively captured in this study, is the decrease in transverse beam stiffness toward the buckling bifurcation point. Studies performed by Tseng and Dugundji (1970) and Virgin and Plaut (2003) verify this phenomena.

\subsubsection{Three Mode Discretization}

For perfect beams, the second mode is never directly activated under symmetric transverse excitation. This is qualitatively easily understood, but also seen quantitatively in the equations of motion for the buckled beam model. For modal response state variable, $q_{m}$, the external forcing term is multiplied by a constant that is the anti-derivative of the $m$ th mode shape. This constant is the projection of the forcing function on the $m$ th mode. For symmetric modes (or even indices), this constant is zero. There can be, however, inter-modal coupling that can excite non-active modes, but the symmetric modes are never directly activated. Even under asymmetric excitation, or imperfect beams, the contribution of the second mode (and all other even

modes) to the beam response is never observed at the beam midpoint. As an additional study, a third mode was added to the discretized buckled beam model. Fig. 6.10 shows the beam response due to the first, second, and third modes, measured at the midpoint, with an initial static deflection of $0.0315 \mathrm{in}$. The response amplitude is normalized by the beam thickness. 


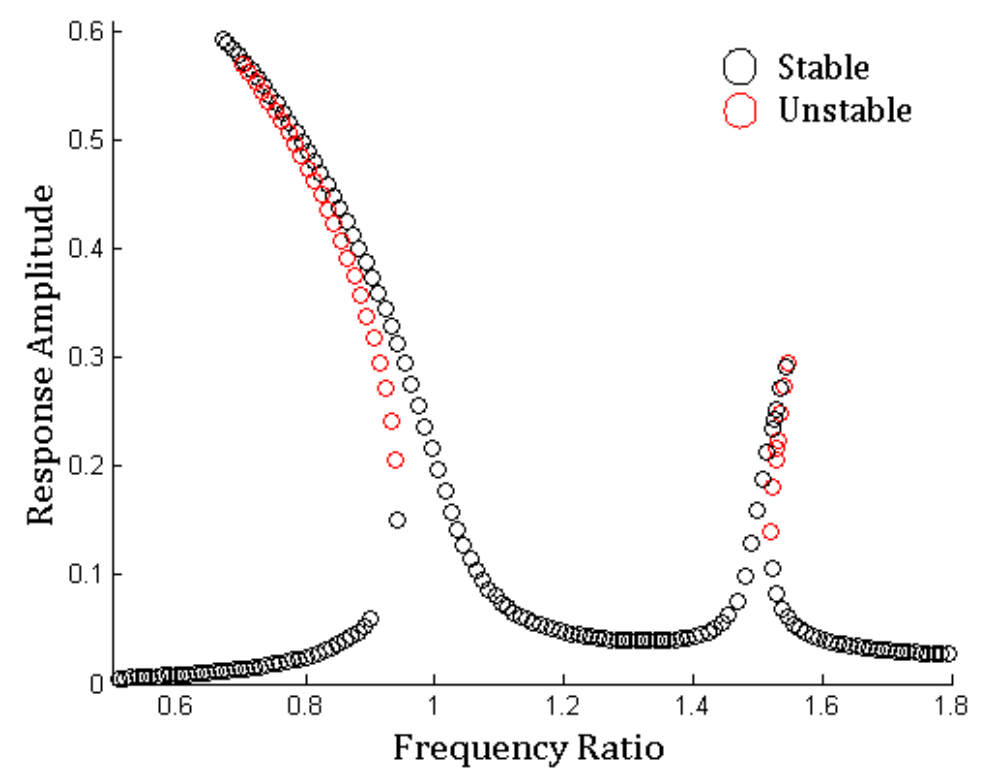

Figure 6.10: Buckled Beam Model (Three Mode Discretization)

In the three mode response, we see no significant change to the response of the first mode. The response amplitude and nonlinearity are no different those seen in the two mode model. The third mode, however demonstrates a nonlinear hardening behavior at its resonance. The modal contribution of each mode to the response at the midpoint can be seen in the linear mode shapes:

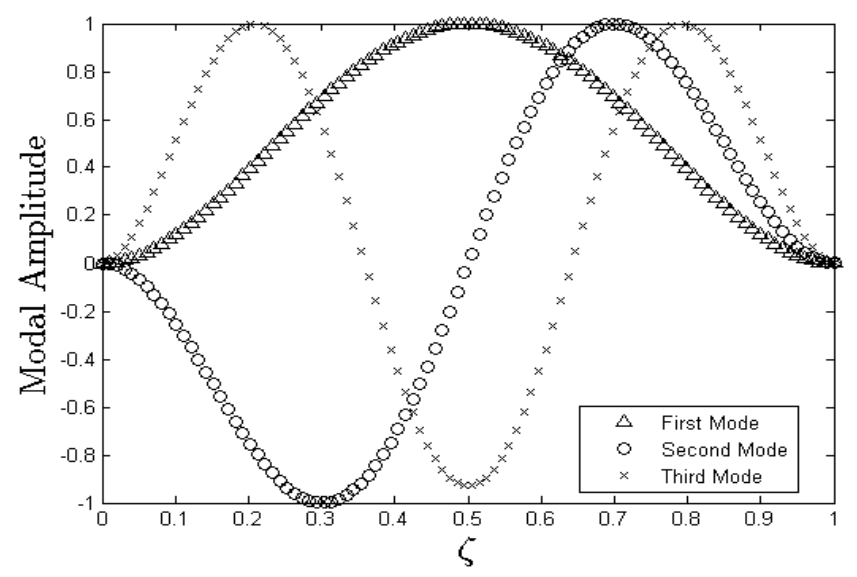

Figure 6.11: First and Third Mode Shapes

We see in the linear mode shapes that at $\zeta=0.5$, there is full contribution of the 
first and third modes, but zero contribution of the second mode. As an additional parametric study, the measurement location, $\zeta$ as well as the static midspan rise due to buckling, $b$ are varied on the three mode buckled beam model. Frequency response plots were generated at $\zeta=[0.8,0.85,0.9]$ and at $b=[0.0315,0.0504,0.063]$ in.. This study is shown in Fig. 6.12. 


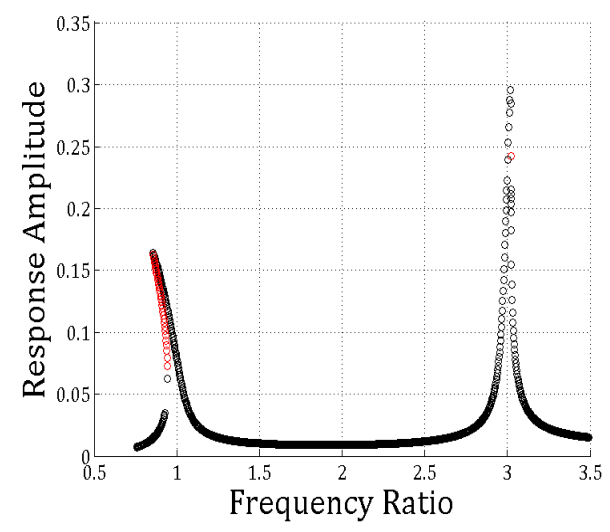

(a) $\zeta=0.8 b=0.063 \mathrm{in}$.

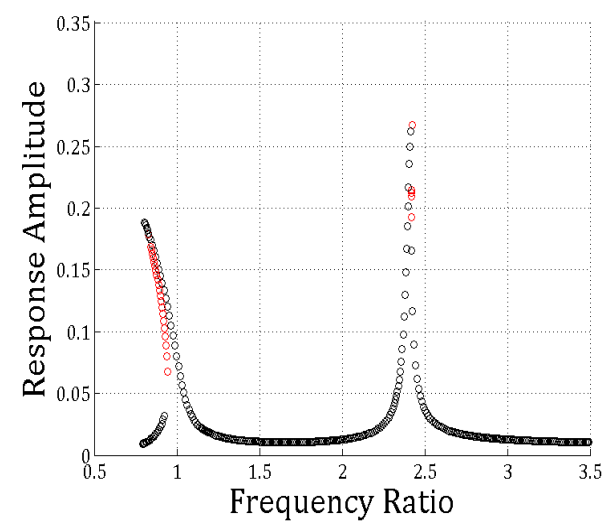

(d) $\zeta=0.8 b=0.0504 i n$.

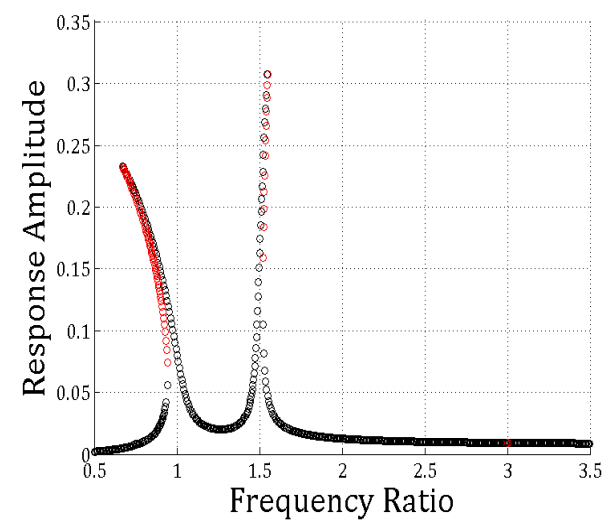

(g) $\zeta=0.8 b=0.0315 i n$.

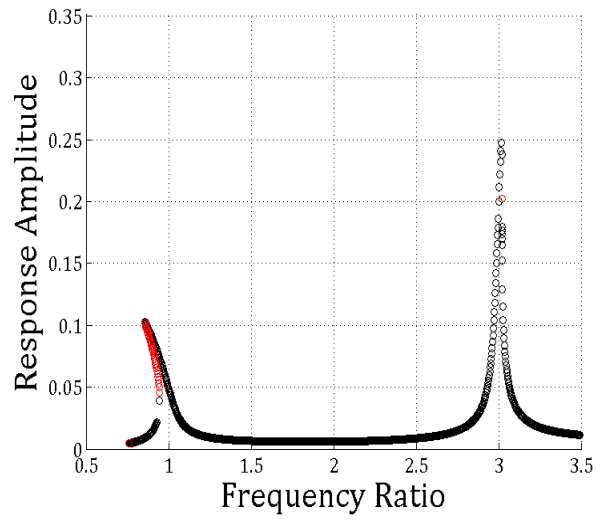

(b) $\zeta=0.85 b=0.063 \mathrm{in}$.

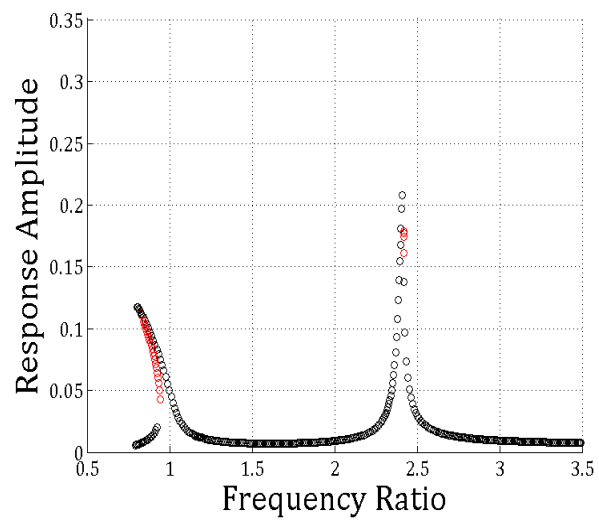

(e) $\zeta=0.85 b=0.0504 i n$.

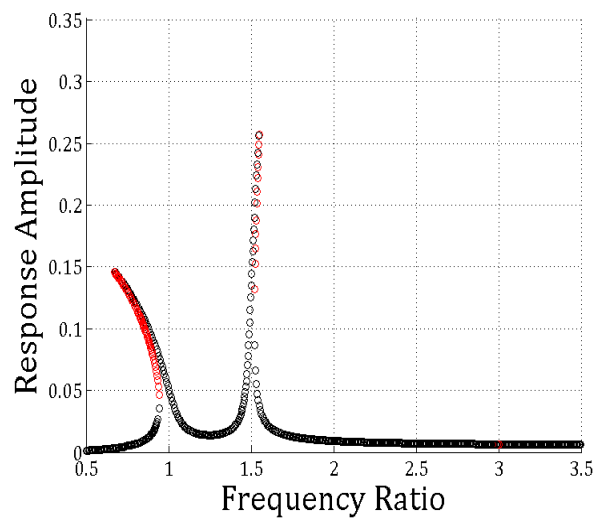

(h) $\zeta=0.85 b=0.0315$ in .

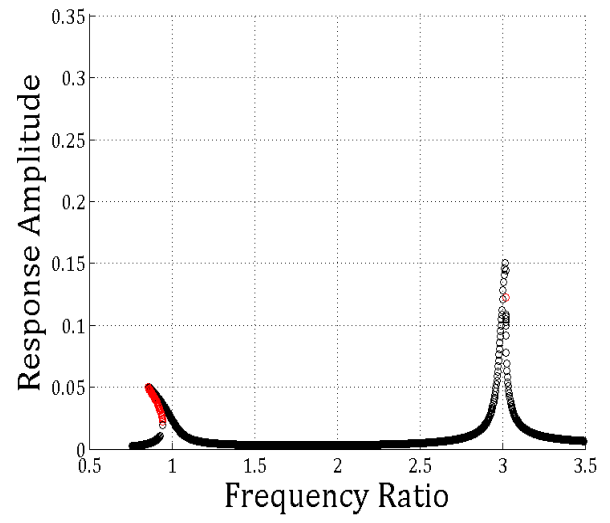

(c) $\zeta=0.9 b=0.063$ in.

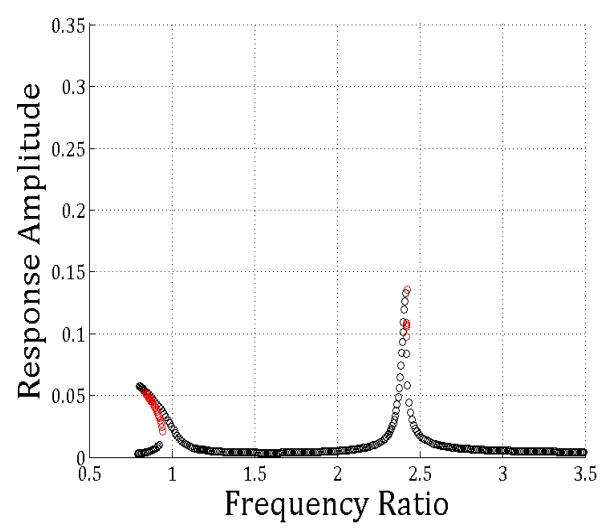

(f) $\zeta=0.9 b=0.0504 \mathrm{in}$.

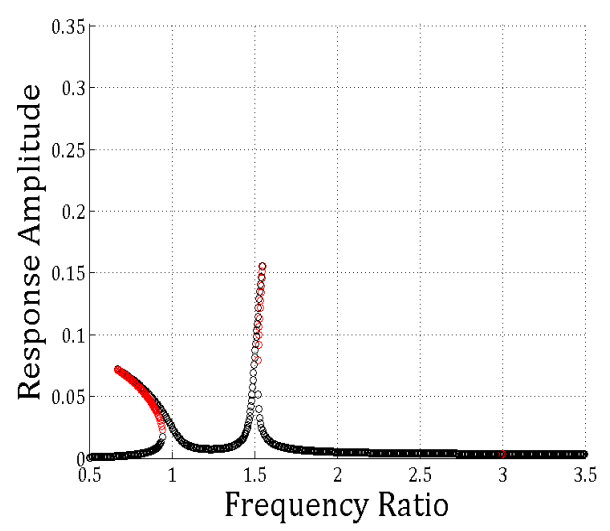

(i) $\zeta=0.9 b=0.0315 \mathrm{in}$.

Figure 6.12: Buckled Beam (3 Mode Discretization) Two Parameter Study 
We see in the two parameter parametric study that there is an increase in response amplitudes of both the first and third mode as the buckling level is reduced, which is consistent with past parametric studies and optimization trials. We also observe that the relative separation between the first and third modes increases as the buckling level is increased. This phenomena has been researched by Lacarbonara (1997), Emam (2002), and Virgin and Plaut (2003). In addition to the modal separation, we also observe the effect of measurement location on the responses. We see that toward $\zeta=0.8$, both vibration modes are amplified. Furthermore, the third mode response amplitude is greater than that of the first mode, throughout this design space.

At this point, an important question arises: is there a more robust, efficient method to obtain the peak amplitude of the beam response subject to various system parameters? Can the methods presented in Chapter V optimize within different design spaces to achieve amplified, periodic, and stable responses? Chapter VII aims to answer this question. 


\section{CHAPTER VII}

\section{Optimization Case Studies}

In this chapter, we aim to first verify the optimization approach presented in Chapter V, by comparing an optimization result with one found via arc length continuation. After the method is verified, we present case studies demonstrating the use of optimization to exploit the nonlinear dynamics of the buckled beam model with harmonic forcing. Specifically, we investigate the effect of the beam geometry (length, width, and thickness), buckling level, base excitation displacement, and measurement location along the beam on the periodic response amplitude of the beam. We then present additional case studies of optimization of the piezoelectric driven beam model investigating the effects of beam and piezoelectric element geometry, buckling level and applied excitation voltage on the periodic beam response.

\subsection{Optimization Method Verification}

In this section, the optimization approach and algorithm presented in Chapter $\mathrm{V}$ is verified. The Scatter Search Optimization Algorithm (SSOA) is used throughout this chapter. The verification approach tests the optimization method's ability to find the necessary design variables that produce the peak response amplitude of the mid-span of the buckled beam (non-hysteresis forcing) model. This is akin to finding the peak solution in Fig. 6.8. Stated formally we aim to maximize the function 


$$
\text { maximize: } f\left(\boldsymbol{q}_{\mathbf{0}}, \Omega\right)=\frac{w(0.5, t)_{\max }-w(0.5, t)_{\min }}{2}
$$

where $\boldsymbol{q}_{\mathbf{0}}$ is the vector of initial state variables in modal coordinates. The right side of Eq. 7.1 is the physical amplitude of the response of the beam measured at the midpoint when subject to the initial state vector, $\boldsymbol{q}_{\mathbf{0}}$. Even though the second mode contributes zero displacement at the midpoint, it contributes to the nonlinear behavior of the first mode, as seen in Chapter VI. As such, the buckled beam, two mode discretization model is used. In order to ensure periodicity of the solution, the nonlinear equality constraint is imposed

$$
H\left(\boldsymbol{q}_{\mathbf{0}}, \Omega\right)=\max \left|\boldsymbol{q}_{\boldsymbol{T}}-\boldsymbol{q}_{\mathbf{0}}\right|<\epsilon
$$

where $\epsilon$ is the convergence tolerance on the shooting function and is set to $10^{-6}$ for all case studies. Additionally, the nonlinear inequality constraint, based on Floquet stability criteria, is imposed to ensure that the response is stable

$$
g\left(\boldsymbol{q}_{\mathbf{0}}, \Omega\right)=\max |\boldsymbol{\rho}|<1
$$

The local solver used by the SSOA is MATLAB's built-in sequential quadratic programming method, which performs function minimization, not maximization. As such the objective function used, computationally, in the SSOA is given as

$$
\text { minimize: }-f\left(\boldsymbol{q}_{\mathbf{0}}, \Omega\right)=-\frac{w(0.5, t)_{\max }-w(0.5, t)_{\min }}{2}
$$

The SSOA was implemented with 2000 diverse trials points, distributed via Latin Hypercube sampling, and 1000 points included in the reference set. Figure 7.1 shows the physical response of the beam after optimization.

Figure 7.1 shows that the optimization solution agrees well with the peak solution 


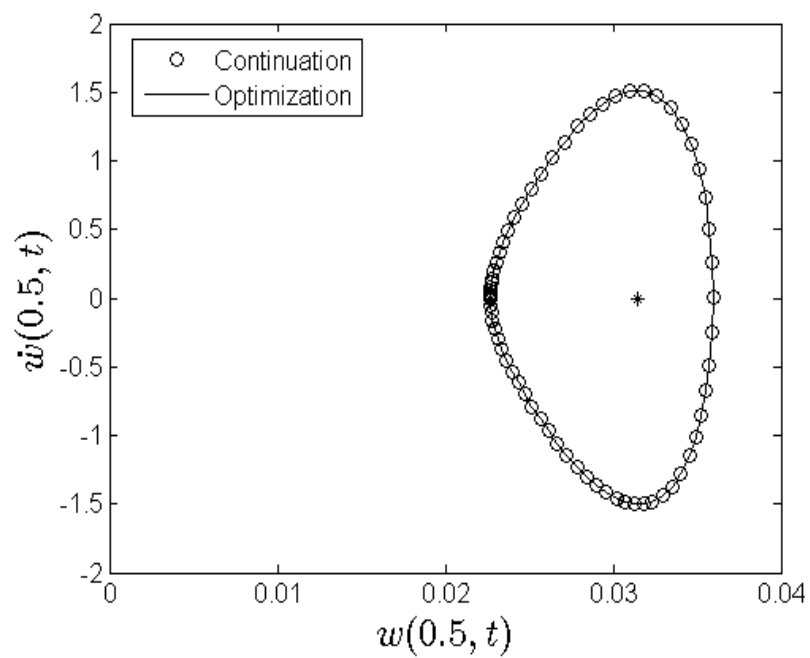

Figure 7.1: Optimization Method Verification (Measured At Beam Midpoint)

found via arc-length continuation. The asterisk in Fig. 7.1 represents the static buckled configuration $(b=0.0315 \mathrm{in})$, and zero on the horizontal axis represents the horizontal unbuckled configuration of the beam. To further compare the two solutions as well as verify the constraint adherence, Table 7.1 compares the solutions found for maximum amplitude of each method.

Table 7.1: Solutions Found Via Optimization and Continuation Methods

\begin{tabular}{lllll}
\hline Design Vector & Optimization & Continuation & Units & Difference (\%) \\
\hline$q_{1_{0}}$ & -0.788159 & -0.784396 & - & 0.4797 \\
$\dot{q}_{1_{0}}$ & -0.00822609 & -0.00822409 & - & 0.02431 \\
$q_{2_{0}}$ & -0.000274449 & -0.000247181 & - & 10.451 \\
$\dot{q}_{2_{0}}$ & -0.384301 & -0.363272 & - & 5.625 \\
$\Omega$ & 15.9840 & 16.0558 & $H z$. & 0.4482 \\
$\max |\boldsymbol{\rho}|$ & 0.9466 & 0.9457 & - & 0.0951 \\
\hline Objective Function & & & & \\
\hline$f\left(\boldsymbol{q}_{\mathbf{0}}, \Omega\right)$ & 0.006644389 & 0.00643774 & in. & 3.159 \\
CPU Time & 28.01 & 50.56 & $s$ & 57.401 \\
\hline
\end{tabular}

Table 7.1 shows excellent agreement between the two methods. Not only were similar results found, but both methods found solutions that satisfied all constraints. Furthermore, the optimization approach proved to be about $45 \%$ computationally 
faster than solution generation via the arc-length continuation method.

Case studies that incorporate several system parameters as variables are explored next. We begin with the harmonically excited, buckled beam model.

\subsection{Optimization Case Studies: Harmonic Forcing}

In this section, several case studies are presented in which the optimization method aims to maximize the nonlinear, buckled beam response amplitude as a function of several design variables and constraints. We begin with the buckled beam (two mode discretization) model with harmonic forcing.

\subsubsection{Case 1: Buckling Level and Beam Width}

In this case study, we aim to maximize the buckled beam response amplitude, measured at the midpoint, while restricting the motion of the beam about one of the buckled configuration (i.e. motion without snap-though). We will use the two mode discretization model here, and not the three mode model, as we are maximizing the amplitude at the beam's midpoint. It was observed in the three mode model's frequency response (in Chapter VI), that the response amplitude at the midpoint due to the third mode is lower than that of the first mode. Furthermore, we saw that the inclusion of the third mode did not significantly affect the response of the first mode (no modal coupling observed). The response of the first mode was, however, influenced by the second mode via modal coupling. As such, the two mode beam model is used.

For this case study, the constraints on periodicity and stability will we applied. We also introduce two design variables to be used in the optimization problem: the initial midspan rise of the center of the beam due to buckling, $b$ and the geometric beam width, $c$. The bounds for the design variables are given in Table 7.2. 
Table 7.2: Case 1 Design Space

\begin{tabular}{lllll}
\hline Variable & Lower Bound & Baseline & Upper Bound & Unit \\
\hline$b$ & 0.0053 & 0.0315 & 0.058 & in. \\
$c$ & 0.25 & 0.5 & 2 & in. \\
\hline
\end{tabular}

Formally, the optimization problem is stated as

$$
\begin{gathered}
\text { maximize: } f\left(\boldsymbol{x}_{\mathbf{0}}, \Omega, \boldsymbol{v}\right) \\
\text { s.t. }\left\{\begin{array}{l}
H\left(\boldsymbol{q}_{\mathbf{0}}, \Omega, \boldsymbol{v}\right)=\max \left|\boldsymbol{q}_{\boldsymbol{T}}-\boldsymbol{q}_{\mathbf{0}}\right|<\epsilon \\
g\left(\boldsymbol{q}_{\mathbf{0}}, \Omega, \boldsymbol{v}\right)=\max |\boldsymbol{\rho}|<1 \\
\min (w(0.5, t))>0
\end{array}\right.
\end{gathered}
$$

The last constraint in Eq. 7.6 ensures non-snap-through behavior as 0 represents the unbuckled configuration of the beam. The SSOA was implemented with 2000 diverse trials points, distributed via Latin Hypercube sampling, and 1000 points included in the reference set. Figure 7.2 shows the result of the optimization.

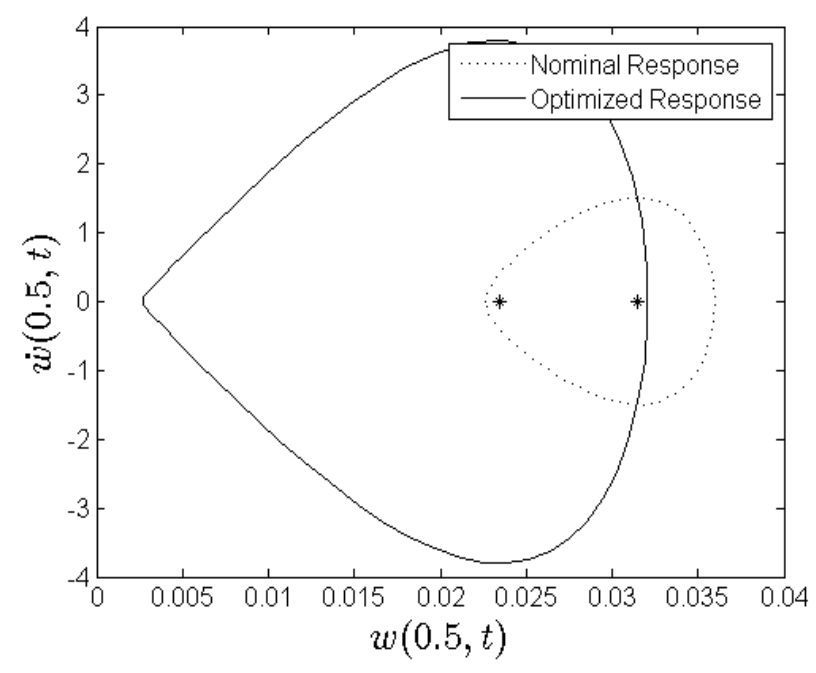

Figure 7.2: Optimization Case 1 Result (Measured at Beam Midpoint)

Qualitatively, Fig. 7.2 shows that the response amplitude of the beam was in- 
creased while remaining within the buckled regime. We observe that by lowering the static rise of the beam, the response amplitude increased. To further explore the results of the optimization, Table 7.3 shows the detailed results.

\begin{tabular}{|c|c|c|}
\hline Design Vector & Value & Unit \\
\hline$q_{1_{0}}$ & -0.974611 & - \\
\hline$\dot{q}_{1_{0}}$ & 1.57574 & - \\
\hline$q_{2_{0}}$ & 0.191891 & - \\
\hline$\dot{q}_{2_{0}}$ & 5.70138 & - \\
\hline$\Omega$ & 12.194 & $H z$. \\
\hline$c$ & 1.4516 & $i n$ \\
\hline$b$ & 0.023456 & in. \\
\hline \multicolumn{3}{|l|}{ Constraints } \\
\hline $\max \left(\left|H\left(\boldsymbol{q}_{\mathbf{0}}, \Omega, \boldsymbol{v}\right)\right|\right)$ & $3.17753 \times 10^{-9}$ & - \\
\hline $\max (|\rho|)$ & 0.99450 & - \\
\hline $\min (w(0.5, t))$ & 0.00265751 & in. \\
\hline \multicolumn{3}{|l|}{ Objective Function } \\
\hline$f\left(\boldsymbol{q}_{\mathbf{0}}, \Omega, \boldsymbol{v}\right)$ & 0.014703 & in. \\
\hline
\end{tabular}

Table 7.3 shows that the maximized solution was found toward lowered values of static midspan rise (toward unbuckling). This trend is consistent with the parametric studies performed in Chapter VI. We also see that the solution was toward a wider beam. Furthermore, we see that the shooting function converged to an acceptable value and Floquet stability was achieved as illustrated by the maximum Floquet multiplier being 0.9945 . The motion was also restricted to the buckled configuration as the minimum displacement was $0.00265751 \mathrm{in}$. Finally, Table 7.3 also shows a $121 \%$ increase in response amplitude when compared to the baseline response of Fig. 7.1.

\subsubsection{Case 2: Beam Geometry, Buckling Level, Excitation Amplitude}

In this case study, we aim to maximize the response amplitude of the buckled beam model as a function of: initial mid span rise, $b$, external forcing displacement amplitude, $W_{B}$, beam thickness, $h$, and beam length, $L$. The initial conditions and 
forcing frequency will also be included in the optimization as usual. The bounds on each design variable are given in Table 7.4.

Table 7.4: Case 2 Design Space

\begin{tabular}{lllll}
\hline Variable & Lower Bound & Baseline & Upper Bound & Unit \\
\hline$b$ & 0.0053 & 0.0315 & 0.058 & in. \\
$W_{B}$ & 0.1 & 0.2 & 0.6 & in. \\
$h$ & 0.007 & 0.021 & 0.0630 & in. \\
$L$ & 9.0 & 18.0 & 27.0 & in. \\
\hline
\end{tabular}

As with the previous case study, we constrain motion to the buckled configuration, and require periodicity and stability of the response. Formally, the optimization problem for this study is stated:

$$
\begin{gathered}
\text { maximize: } f\left(\boldsymbol{q}_{\mathbf{0}}, \Omega, \boldsymbol{v}\right) \\
\text { s.t. }\left\{\begin{array}{l}
H\left(\boldsymbol{q}_{\mathbf{0}}, \Omega, \boldsymbol{v}\right)=\max \left|\boldsymbol{q}_{\boldsymbol{T}}-\boldsymbol{q}_{\mathbf{0}}\right|<\epsilon \\
g\left(\boldsymbol{q}_{\mathbf{0}}, \Omega, \boldsymbol{v}\right)=\max |\boldsymbol{\rho}|<1 \\
\min (w(0.5, t))>0
\end{array}\right.
\end{gathered}
$$

The SSOA was implemented with 2000 diverse trials points, via Latin Hypercube sampling, and 1000 points included in the reference set. Fig. 7.3 shows the result of the optimization.

Figure 7.3 shows that the buckled beam response was greatly amplified because of the additional degrees of freedom given to the design space (the inclusion of additional system parameters). Furthermore, the response obeys the non-snap through constraint but exploits the dynamics to maximize the response. Table 7.5 shows the detailed results of the optimization.

The quantitative results of the optimization show that the shooting function as well as the stability constraints were satisfied. The minimum response displacement was $2.341 \times 10^{-4} \mathrm{in}$. which is close to the bounds on the displacement constraint. 


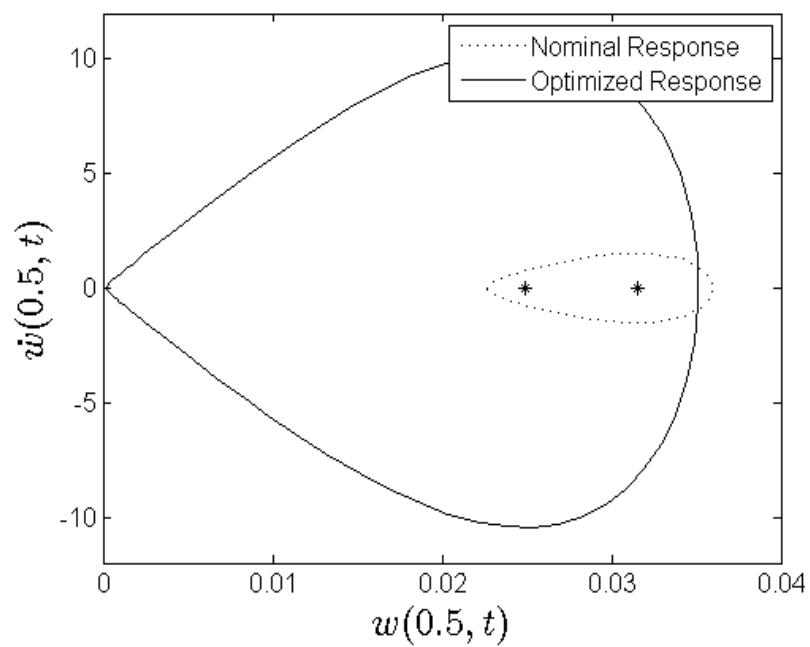

Figure 7.3: Optimization Case 2 Result (Measured at Beam Midpoint)

The response amplitude increased about 166\% from the baseline case. Furthermore, the trends of the beam response with respect to the initial static deflection, $b$, and the beam thickness, $h$, are in a agreement with the parametric studies presented in Chapter VI. Specifically, the optimization found maximum amplitude at higher values of beam thickness (previously explored in section 6.2.2) and at lower values of static rise due to buckling (previously explored in section 6.3.2), with respect to the baseline case. We also see that the beam velocity drastically increases when compared to the baseline case. This is due, in part to the approximately $162 \%$ increase in excitation amplitude.

\subsubsection{Case 3: Snap-Through Dynamics}

In this case study, we will remove the constraint previously applied on the displacement of the beam to stay within the buckled regime. Now, instead, the optimization algorithm will attempt to find the maximum response amplitude within the design space, regardless if snap-through is occurring. The design variables used in this study are the initial static deflection, $b$, the beam thickness, $h$, and the beam length, $L$. 
Table 7.5: Solution Found Via Optimization

\begin{tabular}{lll}
\hline Design Vector & Value & Unit \\
\hline$q_{1_{0}}$ & -0.932588 & - \\
$\dot{q}_{1_{0}}$ & -17.5941 & - \\
$q_{2_{0}}$ & 0.0421334 & - \\
$\dot{q}_{2_{0}}$ & 0.281924 & - \\
$\Omega$ & 21.291 & $H z$. \\
$b$ & 0.0249 & in. \\
$W_{B}$ & 0.28137 & in. \\
$h$ & 0.057638 & in. \\
$L$ & 21.502 & in. \\
\hline Constraints & & \\
\hline max $\left(\left|H\left(q_{0}, \Omega, v\right)\right|\right)$ & $8.721287 \times 10^{-9}$ & - \\
$\max (|\boldsymbol{\rho}|)$ & 0.97320 & - \\
$\min (w(0.5, t))$ & $2.341 \times 10^{-4}$ & in. \\
\hline Objective Function & & \\
\hline$f\left(\boldsymbol{q}_{\mathbf{0}}, \Omega, \boldsymbol{v}\right)$ & 0.0174345 & in. \\
\hline
\end{tabular}

The adjusted optimization problem and constraints thus become

$$
\begin{gathered}
\text { maximize: } f\left(\boldsymbol{x}_{\mathbf{0}}, \Omega, \boldsymbol{v}\right) \\
\text { s.t. }\left\{\begin{array}{l}
H\left(\boldsymbol{x}_{\mathbf{0}}, \Omega, \boldsymbol{v}\right)=\max \left|\boldsymbol{x}_{\boldsymbol{T}}-\boldsymbol{x}_{\mathbf{0}}\right|<\epsilon \\
g\left(\boldsymbol{x}_{\mathbf{0}}, \Omega, \boldsymbol{v}\right)=\max |\boldsymbol{\rho}|<1
\end{array}\right.
\end{gathered}
$$

The bounds on the design variables are given in Table 7.6.

Table 7.6: Case 3 Design Space

\begin{tabular}{lllll}
\hline Variable & Lower Bound & Baseline & Upper Bound & Unit \\
\hline$b$ & 0.0053 & 0.0315 & 0.058 & in. \\
$h$ & 0.007 & 0.021 & 0.0630 & in. \\
$L$ & 9.0 & 18.0 & 27.0 & in. \\
\hline
\end{tabular}

Again, the SSOA was implemented with 2000 diverse trials points, distributed via Latin Hypercube sampling, and 1000 points included in the reference set. Fig. 7.4 shows the result of the optimization. 


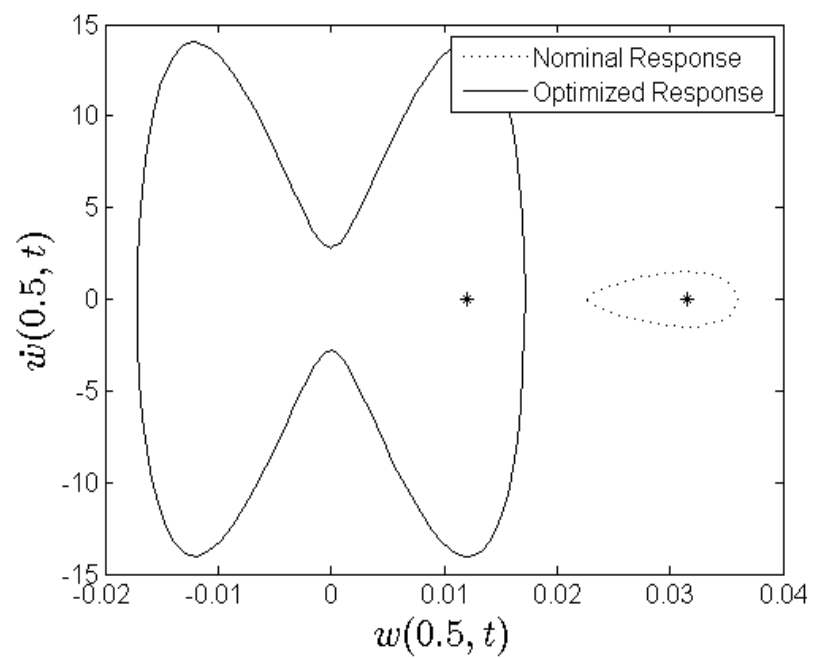

Figure 7.4: Optimization Case 3 Result (Measured at Beam Midpoint)

Fig. 7.4 shows that without the snap through constraint, the optimization algorithm found a solution with larger response amplitude than that of the previous cases by exploiting the nonlinear snap through dynamics. Furthermore, we see a decrease in velocity as the beam crosses the horizontal, as expected. The numerical results for this optimization case study are shown in Table 7.7 .

\begin{tabular}{lll}
\multicolumn{3}{c}{ Table 7.7: Solution Found Via Optimization } \\
\hline Design Vector & Value & Unit \\
\hline$q_{1_{0}}$ & -1.106273 & - \\
$\dot{q}_{1_{0}}$ & -63.85210 & - \\
$q_{2_{0}}$ & -0.00742378 & - \\
$\dot{q}_{2_{0}}$ & 2.938056 & - \\
$\Omega$ & 24.416 & $H z$. \\
$b$ & 0.0121 & in. \\
$h$ & 0.0546 & in. \\
$L$ & 20.495 & in. \\
\hline Constraints & & \\
max $\left(\left|H\left(\boldsymbol{q}_{\mathbf{0}}, \Omega, \boldsymbol{v}\right)\right|\right)$ & $2.45276 \times 10^{-7}$ & - \\
max $(|\boldsymbol{\rho}|)$ & 0.94120 & - \\
\hline Objective Function & & \\
\hline$f\left(\boldsymbol{q}_{\mathbf{0}}, \Omega, \boldsymbol{v}\right)$ & 0.0171724 & in. \\
\hline
\end{tabular}

Table 7.7 shows that the solution for maximum amplitude of this configuration 
is toward a thicker, longer beam, and toward a lower initial static deflection. The shooting function as well as Floquet stability constraints were obeyed leading to a solution with a response amplitude that was roughly $158 \%$ larger than the baseline case.

\subsubsection{Case 4: Measurement Location and Buckling Level}

In this case study, we aim to optimize the response amplitude as a function of the static midspan rise due to buckling, $b$, as well as the measurement/observation location along the beam, $\zeta$. As the second vibration mode is not directly activated by the uniform forcing function (and does not appear at the second natural frequency), we will use the three mode buckled beam model for this case study. The two parameter study explored in Chapter VI gives some a priori knowledge of this design space. The bounds on the design variables are given in Table 7.8 .

Table 7.8: Case 4 Design Space

\begin{tabular}{lllll}
\hline Variable & Lower Bound & Baseline & Upper Bound & Unit \\
\hline$b$ & 0.0053 & 0.0315 & 0.058 & in. \\
$\zeta$ & 0.75 & 0.85 & 0.95 & - \\
\hline
\end{tabular}

Formally, the optimization problem for this study is stated:

$$
\begin{gathered}
\text { maximize: } f\left(\boldsymbol{q}_{\mathbf{0}}, \Omega, \boldsymbol{v}\right) \\
\text { s.t. }\left\{\begin{array}{l}
H\left(\boldsymbol{q}_{\mathbf{0}}, \Omega, \boldsymbol{v}\right)=\max \left|\boldsymbol{q}_{\boldsymbol{T}}-\boldsymbol{q}_{\mathbf{0}}\right|<\epsilon \\
g\left(\boldsymbol{q}_{\mathbf{0}}, \Omega, \boldsymbol{v}\right)=\max |\boldsymbol{\rho}|<1 \\
\min (w(0.5, t))>0
\end{array}\right.
\end{gathered}
$$

Again, the SSOA was implemented with 2000 diverse trials points, distributed via Latin Hypercube sampling, and 1000 points included in the reference set. Figure 7.5 shows the result of the optimization. 


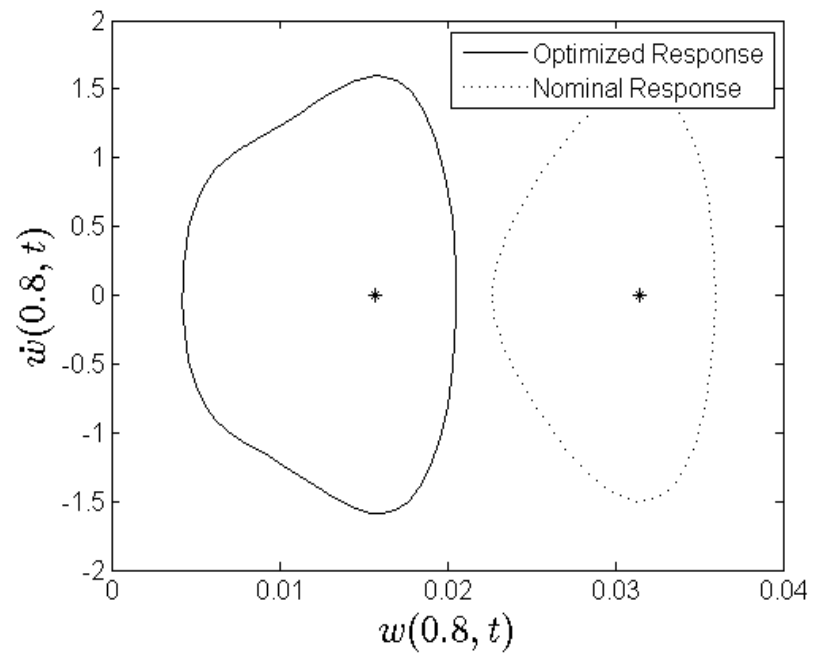

Figure 7.5: Optimization Case 4 Result (Measured at $\zeta=0.8$ )

We see that the optimized response was amplified against the baseline case, even with the bracketed observation location. The nominal response in Fig. 7.5 is the response at the midpoint of the beam and the optimized response is measured at the new observation location. We also see that the shape of the response is qualitatively different than any of the previous trials. This is possibly due to the contribution of the third mode to the total response. Table 7.9 shows the details of this study.

Firstly, we see in the detailed results, that all constraints were satisfied. Secondly, we see that the solution found was toward lower buckling level and at an observation location of $\zeta=0.8$. Furthermore, we see from the initial condition vector that, both the first and third modes were activated at this configuration. The second mode, however, has little contribution. Fig. 7.6 and Fig. 7.7 depict the physical motion of the beam due to each mode at $\zeta=0.8$.

We see that both the first and third mode, do contribute to the overall dynamics of the beam at this observation location. Furthermore, the SSOA was able to find an amplified solution within the bracketed observation location that exploits modal contributions from both the first and third mode. 
Table 7.9: Solution Found Via Optimization

\begin{tabular}{lll}
\hline Design Vector & Value & Unit \\
\hline$q_{1_{0}}$ & -0.392991 & - \\
$\dot{q}_{1_{0}}$ & 32.0606 & - \\
$q_{2_{0}}$ & $1.0398 \times 10^{-4}$ & - \\
$\dot{q}_{2_{0}}$ & $4.3932 \times 10^{-7}$ & - \\
$q_{3_{0}}$ & -0.213973 & - \\
$\dot{q}_{3_{0}}$ & 39.5667 & - \\
$\Omega$ & 31.932 & $H z$. \\
$b$ & 0.01575 & in. \\
$\zeta$ & 0.8 & - \\
\hline Constraints & & \\
\hline $\max \left(\left|H\left(\boldsymbol{q}_{\mathbf{0}}, \Omega, \boldsymbol{v}\right)\right|\right)$ & $1.27696 \times 10^{-8}$ & in. \\
$\max (|\boldsymbol{\rho}|)$ & 0.7137 & - \\
$\min (w(0.5, t))$ & 0.00423719 & in. \\
\hline Objective Function & & \\
\hline$f\left(\boldsymbol{q}_{\mathbf{0}}, \Omega, \boldsymbol{v}\right)$ & 0.00813813 & in. \\
\hline
\end{tabular}

\subsection{Optimization Case Studies: Hysteresis Forcing}

In the remaining case studies, we aim to optimize the piezoelectrically-forced buckled beam (two mode model) as a function of several design variables, and subject to various constraints. Before we begin, however, we must define the piezoelectric model parameters. Low and Guo (1995) modeled the hysteresis forcing associated with G-1195 PZT (Lead Zirconate Titanate) patches. The parameters found were validated experimentally and are shown in Table 7.10.

Figure 7.8 shows the buckled beam model with the piezoelectric patches, where the black boxes represent individual piezoelectric patches. We assume that there is perfect bonding between the patches and the beam. Additionally, we add the respective mass terms $(\rho A)$ and stiffness terms $(E I)$ for the beam and piezoelectric patches in order to account for the added material. Finally, we assume that the force due to a single element is distributed evenly along the length of the patch.

We continue with the optimization case studies. 


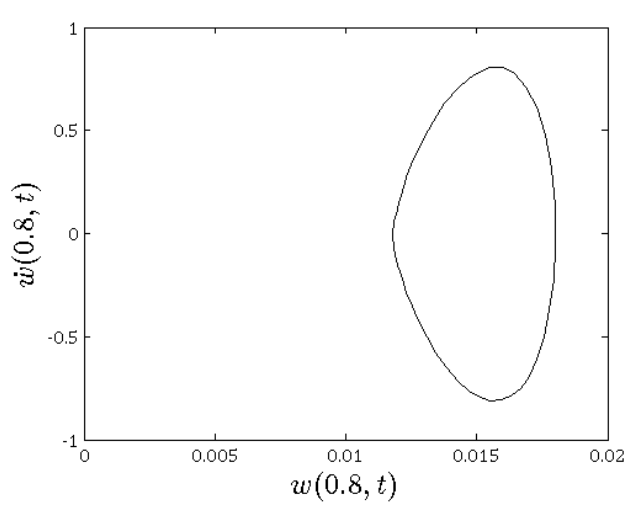

Figure 7.6: First Mode Contribution

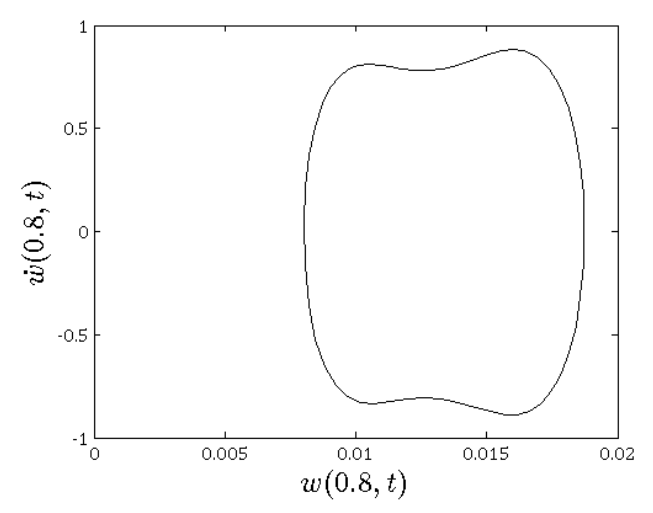

Figure 7.7: Third Mode Contribution

Table 7.10: Piezoelectric Element Parameters

\begin{tabular}{llll}
\hline Dimension & Value & Unit & Description \\
\hline$\alpha$ & $4.297 \times 10^{-1}$ & - & Hysteresis Parameter \\
$\beta$ & $3.438 \times 10^{-2}$ & - & Hysteresis Parameter \\
$d_{31}$ & $7.0866 \times 10^{-9}$ & in. $/ V$ & Piezoelectric Constant \\
$E_{P}$ & $9.1374 \times 10^{6}$ & lb/in. & Piezoelectric Young's Modulus \\
$\gamma$ & $-2.865 \times 10^{-3}$ & - & Hysteresis Parameter \\
$L_{P}$ & 1.5 & in. & Piezoelectric Patch Length \\
$t_{P}$ & 0.0075 & in. & Piezoelectric Patch Thickness \\
$V$ & 100 & $V$ & Applied Voltage \\
$w_{P}$ & 0.375 & in. & Piezoelectric Patch Width \\
\hline
\end{tabular}

\subsubsection{Piezoelectric Beam Nominal Response}

As a baseline case, we will aim to maximize the periodic amplitude of the nonlinear, piezoelectrically-driven, buckled beam model. The constraints will be similar to case study 1 in which we ensured periodicity, stability, and non-snap through response. One additional requirement needed for the piezoelectric cases is periodicity of the hysteresis forcing. Periodicity could be ensured a priori by means of the shooting method. However, several design variables will be used in subsequent optimization trials that will cause the hysteresis forcing to become aperiodic as they are altered. This issue is mitigated by including the hysteresis state variable in the shooting function which will satisfy 


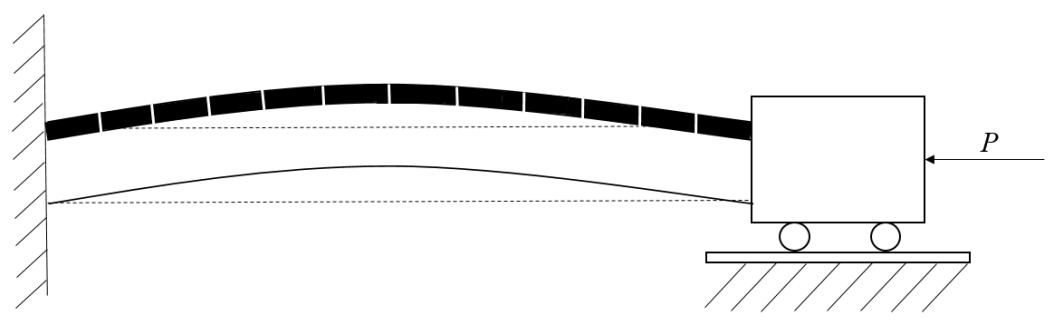

Figure 7.8: Piezoelectric Buckled Beam Model

$$
\left|z_{T}-z_{0}\right|<\epsilon_{z}
$$

which defines the difference between the initial state variable $z_{0}$ and the state variable after one forcing period, $z_{T}$. This state variable will be supplemented to the system state vector, $\boldsymbol{q}_{\mathbf{0}}$, and the hysteresis periodicity error will be supplemented to the shooting function constraint, $H\left(\boldsymbol{q}_{\mathbf{0}}, \Omega\right)$. For the subsequent cases studies, $\epsilon_{z}$ is set to $10^{-6}$. Formally stated, the complete baseline piezoelectric optimization case is given as

$$
\begin{gathered}
\text { maximize: } f\left(\boldsymbol{q}_{\mathbf{0}}, \Omega\right) \\
\text { s.t. }\left\{\begin{array}{l}
H\left(\boldsymbol{q}_{\mathbf{0}}, \Omega\right)=\max \left|\boldsymbol{q}_{\boldsymbol{T}}-\boldsymbol{q}_{\mathbf{0}}\right|<\epsilon \\
g\left(\boldsymbol{q}_{\mathbf{0}}, \Omega\right)=\max |\boldsymbol{\rho}|<1 \\
\min (w(0.5, t))>0
\end{array}\right.
\end{gathered}
$$

The SSOA was implemented with 2000 diverse trials points, distributed via Latin Hypercube sampling, and 1000 points included in the reference set. Figure 7.9 and 7.10 show the result of the optimization.

We observe that the response adhered to the non-snap-through motion as well as apparent periodicity. In addition to the response solution, the optimization algorithm also determined an appropriate value for the initial hysteresis state variable to produce 


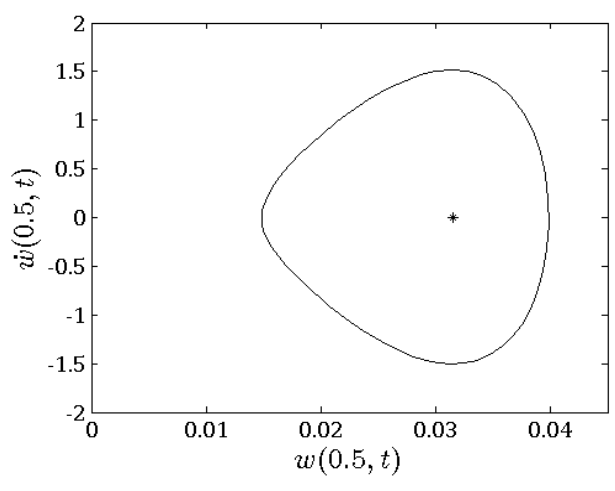

Figure 7.9: Optimized Response

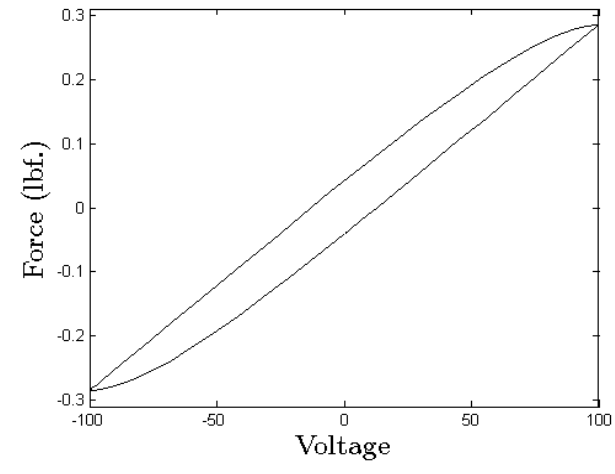

Figure 7.10: Optimized Forcing Profile

Figure 7.11: Optimization Nominal Case Result (Measured at Beam Midpoint)

a periodic forcing function, as shown in Fig. 7.10. The magnitude of the hysteresis is about 10 to $15 \%$ of the motion range. The forcing amplitude is $0.2851 \mathrm{lbf}$. Table 7.11 shows the numerical results of the optimization.

Table 7.11: Solution Found Via Optimization

\begin{tabular}{lll}
\hline Design Vector & Value & Unit \\
\hline$q_{1_{0}}$ & -0.784396 & - \\
$\dot{q}_{1_{0}}$ & -0.00822409 & - \\
$q_{2_{0}}$ & -0.000217181 & - \\
$\dot{q}_{2_{0}}$ & -0.363272 & - \\
$z_{0}$ & $1.26076 \times 10^{-5}$ & - \\
$\Omega$ & 19.174 & $H z$. \\
\hline Constraints & & \\
\hline $\max \left(\left|H\left(q_{0}, \Omega\right)\right|\right)$ & $2.1048 \times 10^{-8}$ & - \\
$\max (|\boldsymbol{\rho}|)$ & 0.99110 & - \\
$\min (w(0.5, t))$ & 0.01482 & in. \\
\hline Objective Function & & \\
\hline$f\left(q_{0}, \Omega\right)$ & 0.012525 & in. \\
\hline
\end{tabular}

Table 7.11 shows that the periodic and stability constraints were satisfied and the response amplitude was found to be $0.01311 \mathrm{in}$.. The periodicity constraint on the hysteresis force was also numerically satisfied. This case study demonstrates that even with several sources of nonlinearity (mid-plane stretching, buckling phenomena, and 
hysteresis, nonlinear forcing), optimization is able to exploit the nonlinear dynamics and achieve a periodic solution that is stable and maximized. Typically, hysteresis phenomena give rise to system energy loss in the form of damping. Even with these nonlinearities, the SSOA found a desired solution.

\subsubsection{Case 5: Piezoelectric Beam Geometry and Buckling Level}

In this case study, we aim to maximize the piezoelectric-forced, buckled beam model's response as a function of the beam thickness, $h$, and initial static midspan rise due to buckling, $b$. This is an interesting case study because the beam thickness impacts the forcing amplitude as well as the overall beam's stiffness.

As before, we apply constraints on periodicity of both the response and the hysteresis forcing function. Floquet stability constraint is also applied. The bounds on the design variables are given in Table 7.12.

Table 7.12: Case 5 Design Space

\begin{tabular}{lllll}
\hline Variable & Lower Bound & Baseline & Upper Bound & Unit \\
\hline$b$ & 0.0053 & 0.0315 & 0.058 & in. \\
$h$ & 0.007 & 0.021 & 0.0630 & in. \\
\hline
\end{tabular}

Furthermore, we restrict response motion to the buckled regime without allowing snap-through dynamics. As such, the optimization problem for this case study is presented:

$$
\begin{gathered}
\text { maximize: } f\left(\boldsymbol{q}_{\mathbf{0}}, \Omega, \boldsymbol{v}\right) \\
\text { s.t. }\left\{\begin{array}{l}
H\left(\boldsymbol{q}_{\mathbf{0}}, \Omega, \boldsymbol{v}\right)=\max \left|\boldsymbol{q}_{\mathbf{T}}-\boldsymbol{q}_{\mathbf{0}}\right|<\epsilon \\
g\left(\boldsymbol{q}_{\mathbf{0}}, \Omega, \boldsymbol{v}\right)=\max |\boldsymbol{\rho}|<1 \\
\min (w(0.5, t))>0
\end{array}\right.
\end{gathered}
$$

The SSOA was implemented with 2000 diverse trials points, distributed via Latin 
Hypercube sampling, and 1000 points included in the reference set. Fig. 7.12 and Fig. 7.13 show the result of the optimization.

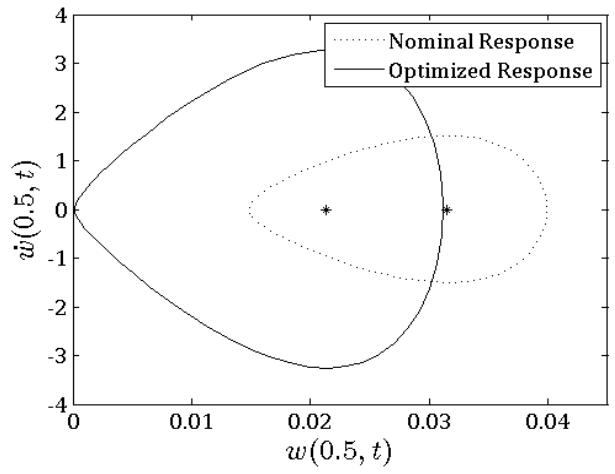

Figure 7.12: Optimized Response

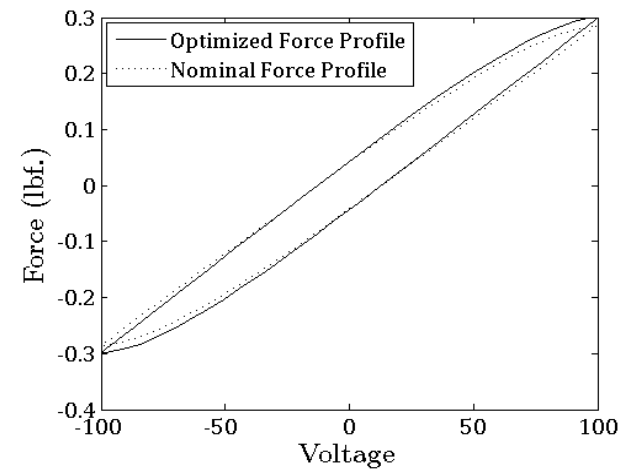

Figure 7.13: Optimized Forcing Profile

Figure 7.14: Optimization Case 5 Result (Measured at Beam Midpoint)

Figure 7.12 shows that the optimization algorithm was able to increase the response amplitude of the piezoelectrically-forced, buckled beam when compared to the nominal response. Figure 7.12 also shows how the snap through constraint was obeyed as the minimum response amplitude is greater than 0. Figure 7.13 shows that the forcing function appears to be periodic as dictated by the optimization constraint. The forcing solution found is nearly identical to the forcing solution found in the previous case study. To investigate the detailed numerical results of this optimization case study, Table 7.13 shows the solution.

We see in Table 7.13 that the response periodicity, forcing periodicity, response stability, and non-snap-though dynamics constraints were all numerically satisfied. Furthermore, the results of this case study follow the trends seen in the parametric studies in Chapter VI. Specifically, the optimized solution was toward greater beam thickness and lower buckling level. The response saw a $23 \%$ increase in periodic amplitude when compared to the baseline, with these optimized parameters. 
Table 7.13: Solution Found Via Optimization

\begin{tabular}{lll}
\hline Design Vector & Value & Unit \\
\hline$q_{1_{0}}$ & -0.955814 & - \\
$\dot{q}_{1_{0}}$ & -0.0408608 & - \\
$q_{2_{0}}$ & 0.0000770798 & - \\
$\dot{q}_{2_{0}}$ & -0.204790 & - \\
$z_{0}$ & $1.27241 \times 10^{-5}$ & - \\
$\Omega$ & 20.159 & $H z$. \\
$b$ & 0.02133 & $i n$. \\
$h$ & 0.02482 & $i n$. \\
\hline Constraints & & \\
\hline $\max \left(\left|H\left(\boldsymbol{q}_{\mathbf{0}}, \Omega, \boldsymbol{v}\right)\right|\right)$ & $1.43802 \times 10^{-7}$ & - \\
$\max (|\boldsymbol{\rho}|)$ & 0.98310 & - \\
$\min (w(0.5, t))$ & $1.40095 \times 10^{-4}$ & $i n$. \\
\hline Objective Function & & \\
\hline$f\left(\boldsymbol{q}_{\mathbf{0}}, \Omega, \boldsymbol{v}\right)$ & 0.0155101 & - \\
\hline
\end{tabular}

\subsubsection{Case 6: Piezoelectric Element Thickness}

In this case study, the thickness of the piezoelectric element layer, $t_{P}$ will be used as design variables to maximize the buckled beam response amplitude. This design variable influences the buckled beam dynamics in two ways. First, the piezoelectric layer thickness has a direct affect on the transverse force output from the elements. Secondly, as the piezoelectric elements have been included as additional mass and stiffness in the model, the their thicknesses also have an effect on the rigidity of the beam. The bounds on the piezoelectric beam thickness are given in Table 7.14. As

Table 7.14: Case 6 Design Space

\begin{tabular}{lllll}
\hline Variable & Lower Bound & Baseline & Upper Bound & Unit \\
\hline$t_{P}$ & 0.0025 & 0.0075 & 0.0225 & in. \\
\hline
\end{tabular}

an aside-in practice, the design space in Table 7.14 may not be feasible, as available piezoelectric elements might not have dimensions within this range. Regardless, we will proceed with the case studies as the purpose of this research is to demonstrate the exploitation of the nonlinear dynamics of the system via optimization. The piezo- 
electric elements simply provide additional degrees of freedom and nonlinearity to the already complex buckled beam system. Practical implementation of these methods would require careful selection of design variable ranges and bounds.

Continuing, the formalized optimization problem for this case study is presented as:

$$
\begin{gathered}
\text { maximize: } f\left(\boldsymbol{q}_{\mathbf{0}}, \Omega, \boldsymbol{v}\right) \\
\text { s.t. }\left\{\begin{array}{l}
H\left(\boldsymbol{q}_{\mathbf{0}}, \Omega, \boldsymbol{v}\right)=\max \left|\boldsymbol{q}_{\boldsymbol{T}}-\boldsymbol{q}_{\mathbf{0}}\right|<\epsilon \\
g\left(\boldsymbol{q}_{\mathbf{0}}, \Omega, \boldsymbol{v}\right)=\max |\rho|<1 \\
\min (w(0.5, t))>0
\end{array}\right.
\end{gathered}
$$

The SSOA was implemented with 2000 diverse trials points, distributed via Latin Hypercube sampling, and 1000 points included in the reference set. Fig. 7.15 and Fig. 7.16 show the result of the optimization.

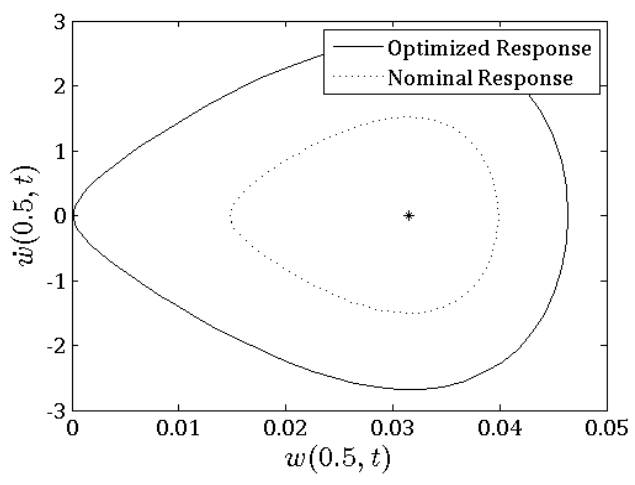

Figure 7.15: Optimized Response

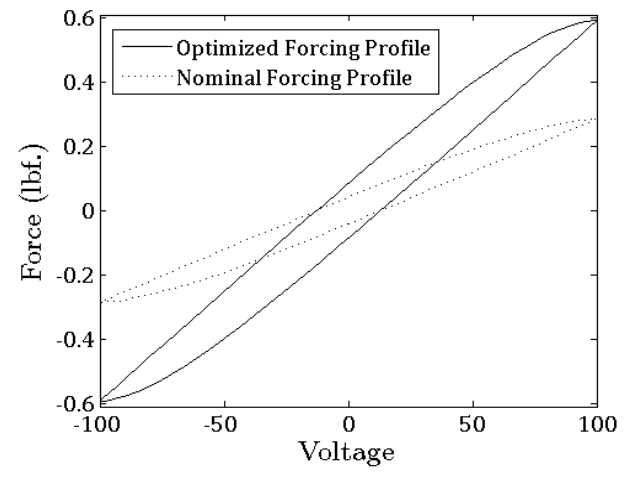

Figure 7.16: Optimized Forcing Profile

Figure 7.17: Optimization Case 6 Result (Measured at Beam Midpoint)

Firstly, we observe that the optimization algorithm was able to maximize the response of the piezoelectric-forced, buckled beam. More importantly however, in Fig. 7.16, we see how the optimum piezoelectric material properties altered the forcing profile. Not only was the force amplitude output increased, but the magnitude of the nonlinear hysteresis behavior was also increased, while remaining periodic. The 
magnitude of the response amplification is not as large as many of the previous cases. Table 7.15 shows the detailed result of the optimization.

\begin{tabular}{lll}
\multicolumn{3}{c}{ Table 7.15: Solution Found Via Optimization } \\
\hline Design Vector & Value & Unit \\
\hline$q_{1_{0}}$ & -0.861213 & - \\
$\dot{q}_{1_{0}}$ & -3.212784 & - \\
$q_{2_{0}}$ & -0.003631206 & - \\
$\dot{q}_{2_{0}}$ & 1.088524 & - \\
$z_{0}$ & $1.16785 \times 10^{-5}$ & - \\
$\Omega$ & 14.169 & $H z$. \\
$t_{P}$ & 0.0155686 & in. \\
\hline Constraints & & \\
\hline $\max \left(\left|H\left(\boldsymbol{q}_{\mathbf{0}}, \Omega, \boldsymbol{v}\right)\right|\right)$ & $7.9689646 \times 10^{-7}$ & - \\
$\max (|\boldsymbol{\rho}|)$ & 0.97820 & - \\
$\min (w(0.5, t))$ & 0.0001447 & in. \\
\hline Objective Function & & in. \\
\hline$f\left(\boldsymbol{q}_{\mathbf{0}}, \Omega, \boldsymbol{v}\right)$ & 0.023262 & \\
\hline
\end{tabular}

From Table 7.15, see that all the applied constraints were satisfied. The solution was toward a thicker piezoelectric element layer. Furthermore, we see a $85 \%$ increase in response amplitude when compared to the piezoelectric baseline case study.

\subsubsection{Case 7: Piezoelectric Snap-Through Dynamics}

As a final comprehensive case study, we aim to maximize the piezoelectricallyforced, buckled beam system as a function of the piezoelectric layer thickness, $t_{P}$, the applied excitation voltage, $V$, the initial static deflection due to buckling, $b$, and the beam thickness, $h$. Furthermore, we will not restrict the motion of the beam (thereby allowing snap-through, if feasible). The bounds on the design variables are given in Table 7.16.

As always, we add constraints to the optimization problem to ensure response and force periodicity, as well as dynamic Floquet stability of the response: 
Table 7.16: Case 7 Design Space

\begin{tabular}{lllll}
\hline Variable & Lower Bound & Baseline & Upper Bound & Unit \\
\hline$t_{P}$ & 0.0025 & 0.0075 & 0.0225 & in. \\
$V$ & 50 & 100 & 150 & V \\
$b$ & 0.0053 & 0.0315 & 0.058 & in. \\
$h$ & 0.0070 & 0.021 & 0.0630 & in. \\
\hline
\end{tabular}

$$
\begin{gathered}
\text { maximize: } f\left(\boldsymbol{q}_{\mathbf{0}}, \Omega, \boldsymbol{v}\right) \\
\text { s.t. }\left\{\begin{array}{l}
H\left(\boldsymbol{q}_{\mathbf{0}}, \Omega, \boldsymbol{v}\right)=\max \left|\boldsymbol{q}_{\boldsymbol{T}}-\boldsymbol{q}_{\mathbf{0}}\right|<\epsilon \\
g\left(\boldsymbol{q}_{\mathbf{0}}, \Omega, \boldsymbol{v}\right)=\max |\boldsymbol{\rho}|<1
\end{array}\right.
\end{gathered}
$$

The SSOA was implemented with 2000 diverse trials points, distributed via Latin Hypercube sampling, and 1000 points included in the reference set. Fig. 7.18 and Fig. 7.19 show the result of the optimization.

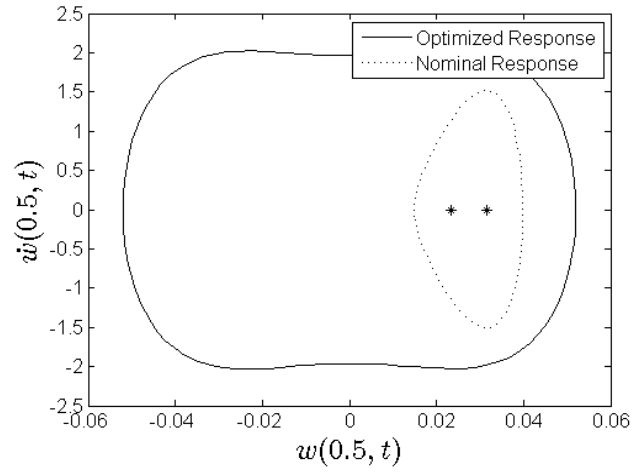

Figure 7.18: Optimized Response

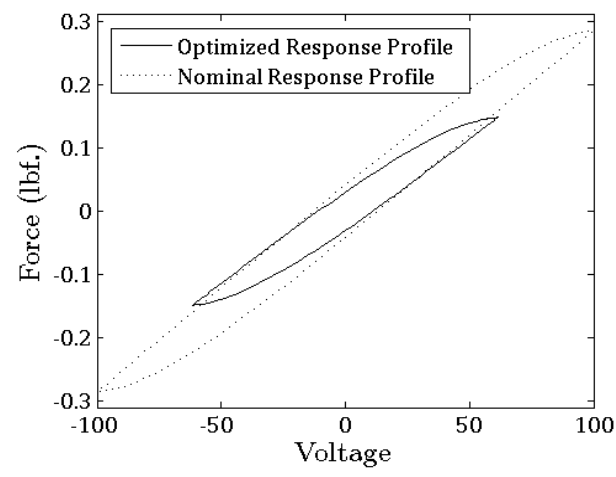

Figure 7.19: Optimized Forcing Profile

Figure 7.20: Optimization Case 7 Result (Measured at Beam Midpoint)

Fig. 7.18 shows that the optimization algorithm took advantage of the relaxed constraint on snap through motion and subsequently more than doubled the response amplitude when compared to the baseline. Even more interesting, however is the optimized force profile. Unlike the previous cases, the optimization method maximized 
the response amplitude without increasing the input force amplitude. The magnitude of the hysteresis stayed relatively unchanged. Table 7.17 shows the detailed result of this case study.

Table 7.17: Solution Found Via Optimization

\begin{tabular}{lll}
\hline Design Vector & Value & Unit \\
\hline$q_{1_{0}}$ & 0.982307 & - \\
$\dot{q}_{1_{0}}$ & -61.347 & - \\
$q_{2_{0}}$ & 0.999680 & - \\
$\dot{q}_{2_{0}}$ & 0.0325905 & - \\
$\Omega$ & 11.730 & $H z$. \\
$t_{P}$ & 0.0055 & $i n$. \\
$V$ & 61.449 & $V$ \\
$b$ & 0.0234 & in. \\
$h$ & 0.01960 & in. \\
\hline Constraints & & \\
\hline max $\left(\left|H\left(\boldsymbol{q}_{\mathbf{0}}, \Omega, \boldsymbol{v}\right)\right|\right)$ & $1.653815 \times 10^{-7}$ & - \\
$\max (|\boldsymbol{\rho}|)$ & 0.99130 & - \\
\hline Objective Function & & \\
\hline$f\left(\boldsymbol{q}_{\mathbf{0}}, \Omega, \boldsymbol{v}\right)$ & 0.052016 & in. \\
\hline
\end{tabular}

From Table 7.17 , we see that the stability and periodicity constraints were satisfied. We also see a possible explanation for increased response amplitude with lowered forcing; the initial velocity of the first generalized coordinate. At -61.34 , this starting value might have provided enough energy to induce snap through. Regardless of the reason, this case study highlights the ability to find solutions in different areas of a design space than expected. The response amplitude was found to be $0.052016 \mathrm{in}$. which is around $315 \%$ greater than the piezoelectric baseline case study.

\subsection{Summary}

In this chapter, optimization case studies demonstrated the ability of the optimization method to maximize the response of the nonlinear beam models, subject to several different sources of nonlinearity. The solutions generated by the method were 
not only amplified, but also periodic and dynamically stable. System parameters with varying levels of influence on the beam response were used as design variables in the optimization problems. Table 7.18 summarizes the results of each of the case studies presented.

Table 7.18: Optimization Case Studies Summary

\begin{tabular}{|c|c|c|c|}
\hline Case & Design Variables & Constraints & $\%$ Amplification \\
\hline \multicolumn{4}{|c|}{ Harmonic Excitation } \\
\hline \multirow[t]{3}{*}{ Case 1} & Buckling Level, $b$ & Response Periodicity & 121 \\
\hline & Beam Width, $c$ & Stability & \\
\hline & & Buckled Regime & \\
\hline \multirow[t]{4}{*}{ Case 2} & Buckling Level, $b$ & Response Periodicity & 166 \\
\hline & Beam Thickness, $h$ & Stability & \\
\hline & Beam Length , $L$ & Buckled Regime & \\
\hline & Base Displacement, $W_{B}$ & & \\
\hline \multirow[t]{3}{*}{ Case 3} & Buckling Level, $b$ & Response Periodicity & 158 \\
\hline & Beam Thickness, $h$ & Stability & \\
\hline & Beam Length, $L$ & & \\
\hline \multirow[t]{3}{*}{ Case 4} & Buckling Level, $b$ & Response Periodicity & 38 \\
\hline & Measurement Location, $\zeta$ & Stability & \\
\hline & & Buckled Regime & \\
\hline \multicolumn{4}{|c|}{ Nonlinear Excitation } \\
\hline \multirow[t]{4}{*}{ Case 5} & Buckling Level, $b$ & Response Periodicity & 23 \\
\hline & Beam Thickness, $h$ & Force Periodicity & \\
\hline & & Stability & \\
\hline & & Buckled Regime & \\
\hline \multirow[t]{4}{*}{ Case 6} & Piezoelectric Thickness, $t_{P}$ & Response Periodicity & 85 \\
\hline & & Force Periodicity & \\
\hline & & Stability & \\
\hline & & Buckled Regime & \\
\hline \multirow[t]{5}{*}{ Case 7} & Buckling Level, $b$ & Response Periodicity & 315 \\
\hline & Piezoelectric Thickness, $t_{P}$ & Force Periodicity & \\
\hline & Voltage, $V$ & Stability & \\
\hline & Buckling Level, $b$ & & \\
\hline & Beam Thickness, $h$, & & \\
\hline
\end{tabular}

For each case study, we see amplification of the response when compared to the nominal cases. The majority of the harmonic forcing case studies found greater response amplitudes than that of the nonlinear forcing studies. This is due, in part, to 
the number of system parameters included in each study, as the harmonic forcing case studies had more system parameters than the nonlinear forcing studies, on average. The results of the case studies were achieved without iteratively sweeping the design space with arc-length continuation applications. Recasting the shooting function and Floquet stability criteria as nonlinear inequalities in the optimization problems allowed traditional optimization strategies to find the desired responses. 


\section{CHAPTER VIII}

\section{Conclusions}

Beam models with nonlinearities due to large beam deflection, dynamic buckling, and piezoelectric material properties have been developed and simulated. Continuation methods have been implemented to explore the nonlinear responses throughout different excitation frequencies. Parametric studies were also performed, giving insight into areas of possible exploitation of the dynamics for design benefit. An optimization approach was formulated that recasts several nonlinear analysis tools as nonlinear constraints in an optimization problem. The constraints ensure periodicity as well as dynamic stability. Finally, case studies were presented that exploit the dynamics of various beam systems using several system parameters and incorporating different sources of nonlinearity.

The nonlinear, analytical model of an Euler Bernoulli beam with large transverse deflection was validated using published experimental data, and required one mode discretization. The nonlinear mid-plane stretching due to large deflection created a cubic term in the governing differential equations giving rise to nonlinear hardening, frequency shifting, jump phenomena, and hysteresis. A parametric study on beam thickness showed that within the studied range of thicknesses, thicker beams lead to more nonlinear, amplified response amplitudes. This amplification was due to the increased coefficient of the nonlinear term in the governing discretized equations. 
The nonlinear, buckled beam, analytical model was also validated against published experimental data, and required two modes in the discretization process for adequate convergence. Both quadratic and cubic terms appear in the governing equations due to mid-plane stretching as well as nonlinear buckling. Nonlinear softening, frequency shifting, jump phenomenon, and hysteresis were observed in the frequency response plots. A parametric study on axial load (post buckling) was performed exploring the effect of the static rise of the midspan of the beam on the dynamic, periodic response. The results of the study showed that decreasing the axial load toward unbuckling lead to increases the response amplitude. Decreased stability was also seen as the axial load was lowered. In an additional parametric study, a third mode was added to the model and a study on the effect of the axial load as well as measurement location along the beam was performed. A separation of the first and third modes was seen as the static rise of the midspan of the beam was increased.

Optimization studies were performed to maximize the nonlinear dynamic response amplitudes of the buckled beam models, subject to several design variables. The optimization method was verified by comparing the peak response found via optimization to the solution found via continuation. The optimized solution agreed very well with the continuation solution and solved the problem in about half the computation time. Recasting the shooting method and Floquet stability into nonlinear constraints, the maximized solutions were forced to be periodic and stable. A Scatter Search Optimization Algorithm was used as the optimization tool and successfully demonstrated the ability to exploit and maximize the buckled beam's nonlinear response by using the axial load, beam geometry, measurement locations, as well as the external forcing amplitude as design variables. Limiting or permitting beam translation across the horizontal (limiting or permitting snap-through) was governed by means of an additional inequality constraint.

Optimization trials were also performed on the piezoelectrically-forced, buckled 
beam model with the same objective: to maximize the nonlinear response amplitude. In addition to the periodicity and stability constraints, an additional constraint was imposed to ensure periodicity of the nonlinear hysteresis forcing trajectory of the piezoelectric elements. The nominal response trial found a baseline solution that was stable as well as had a periodic forcing profile. Beam geometry, axial load, piezoelectric element thickness, and applied excitation voltage were used as design variables in different optimization studies. Similar to the non-piezoelectric beam, the solutions of each case study were amplified, periodic, stable. The hysteresis forcing profiles were all periodic as well.

\section{Recommendations for Future Work}

Additional investigation into the inclusion of the piezoelectric elements is needed. At this point, nonlinear stiffness characteristics of the piezoelectric elements is not included in the model. Not only would this consideration improve model accuracy, but would also add more nonlinearity and dynamic coupling into the system. Furthermore, physical dynamic actuation experiments should be performed on piezoelectric elements and the hysteresis model should be optimized to agree with the tested results.

Optimization trials on the size, shape, and location of the piezoelectric elements should be performed. While the case studies presented in this work highlight optimization on the piezoelectric forcing function, it may be more feasible to alter the size

or placement of the elements instead of their mechanical properties. This task, along with the previous recommendation, create discontinuous piezo-beam dimensions and material properties causing the need to modify the governing equations.

Currently, the Scatter Search Optimization Algorithm performs the global seed evaluations and the local searches sequentially. The implementation of parallel com- 
putation of the local searches would drastically reduce computation time. This reduction in computation time would allow for more design variables, wider bounds on those variables, and the potential to find better, unexpected solutions.

Additionally, constraints on different mechanical or dynamic properties should be imposed on the optimization problems. In engineering applications, the amplified dynamic responses might be achieved at the cost of higher mechanical stress or fatigue. Imposing maximum allowable stress constraints on the optimization problems would help ensure longevity of the system.

Finally, this work lays the foundation for our approach to be used in numerous other applications. Nonlinear, periodic, systems exist in other mechanical systems (aside from buckled beams), as well as biological and chemical systems and our approach may prove to be a suitable tool for achieving desired responses.

The goal of this research was to develop and demonstrate a method to optimize and exploit the nonlinear dynamics of buckled beam models. Numerical, nonlinear dynamic analysis tools were recast as optimization parameters and constraints to be used by the optimization algorithm to find maximized, periodic, stable responses. The method was not only successful, but also did not waste computational resources on large design space sweeps or on iterative shooting method refinements. The approach uses a widely-understood and available gradient-based solver to iterate in the direction of increased response, periodicity, and stability. The demonstrated case studies highlight the ability of this approach to solve problems with several sources of nonlinearity (both in the dynamic system and in the input excitation) and numerous design variables. 
APPENDICES 


\section{APPENDIX A}

\section{Closed Form Frequency Response}

$$
X=\frac{f_{0}}{\omega_{n}^{2} \sqrt{\left(1-r^{2}\right)^{2}+(2 \zeta r)^{2}}}
$$

where $X$ is the response amplitude, $f_{0}$ is the forcing amplitude, $\omega_{n}$ is the natural frequency, $r$ is $\frac{\omega}{\omega_{n}}$, and $\zeta$ is the damping ratio. 


\section{APPENDIX B}

\section{Beam Model Integration Constants}

Table B.1: Buckled Beam Model Integration Constant Values

\begin{tabular}{llll}
\hline$A_{111}=-35.758$ & $A_{212}=-1.4512 \times 10^{-9}$ & $B_{1221}=-5.0915 \times 10^{-10}$ & $B_{2121}=-5.1022 \times 10^{-10}$ \\
$A_{121}=0.00023375$ & $A_{222}=0.00091901$ & $B_{1212}=-5.0915 \times 10^{-10}$ & $B_{2112}=-5.1022 \times 10^{-10}$ \\
$A_{112}=0.00022591$ & $B_{1111}=-11.90$ & $B_{1122}=-49.331$ & $B_{2221}=0.00032328$ \\
$A_{122}=-49.41$ & $B_{1211}=0.000077693$ & $B_{1222}=0.00032206$ & $B_{2212}=0.00032328$ \\
$A_{211}=0.00022986$ & $B_{1121}=0.000077988$ & $B_{2111}=0.000077855$ & $B_{2122}=0.00032273$ \\
$A_{221}=-1.4512 \times 10^{-9}$ & $B_{1112}=0.000077988$ & $B_{2211}=-49.331$ & $B_{2222}=-204.49$ \\
\hline
\end{tabular}




\section{BIBLIOGRAPHY}




\section{BIBLIOGRAPHY}

Abou-Rayan, A., A. Nayfeh, D. Mook, and M. Nayfeh (1993), Nonlinear response of a parametrically excited buckled beam, Nonlinear Dynamics, 4(5), 499-525.

Barari, A., H. Kaliji, M. Ghadimi, and G. Domairry (2011), Non-linear vibration of euler-bernoulli beams, Latin American Journal of Solids and Structures, 8(2), 139-148.

Bashash, S., and N. Jalili (2007), Intelligence rules of hysteresis in the feedforward trajectory control of piezoelectrically-driven nanostagers, Journal of Micromechanics and Microengineering, $17(2), 342$.

Besselink, B., A. Shukla, R. Fey, and H. Nijmeijer (2007), Classification of periodic solutions in a single-degree-of-freedom system with backlash, in ASME 2007 International Design Engineering Technical Conferences and Computers and Information in Engineering Conference, pp. 173-182, American Society of Mechanical Engineers.

Brokate, M., and J. Sprekels (1996), Hysteresis operators, in Hysteresis and Phase Transitions, pp. 22-121, Springer.

Brzeski, P., P. Perlikowski, and T. Kapitaniak (2014), Numerical optimization of tuned mass absorbers attached to strongly nonlinear duffing oscillator, Communications in Nonlinear Science and Numerical Simulation, 19(1), 298-310.

Chang, T.-P. (2012), Nonlinear vibration analysis of a fixed-fixed beam under oscillating axial load and vibrating magnetic field, Journal of Theoretical and Applied Mechanics, 50, 441-453.

Cowper, G. R. (1968), On the accuracy of timoshenkos beam theory, Journal of the Engineering Mechanics Division, 94(6), 1447-1454.

DeVoe, D. L., and A. P. Pisano (1997), Modeling and optimal design of piezoelectric cantilever microactuators, Journal of Microelectromechanical systems, 6(3), 266270.

Dick, A. J. (2013), Characterizing effective d31 values for pzt from the nonlinear oscillations of clamped-clamped micro-resonators, Strojniški vestnik-Journal of Mechanical Engineering, 59(1), 50-55. 
Donoso, A., and O. Sigmund (2009), Optimization of piezoelectric bimorph actuators with active damping for static and dynamic loads, Structural and Multidisciplinary Optimization, 38(2), 171-183.

Dugundji, J. (1971), Nonlinear vibrations of a buckled beam under harmonic excitation1.

Egea, J. A., M. Rodríguez-Fernández, J. R. Banga, and R. Martí (2007), Scatter search for chemical and bio-process optimization, Journal of Global Optimization, $37(3), 481-503$.

Egea, J. A., D. Henriques, T. Cokelaer, A. F. Villaverde, A. MacNamara, D.-P. Danciu, J. R. Banga, and J. Saez-Rodriguez (2014), Meigo: an open-source software suite based on metaheuristics for global optimization in systems biology and bioinformatics, BMC bioinformatics, 15(1), 136.

Eggborn, T. (2003), Analytical models to predict power harvesting with piezoelectric materials.

Elshurafa, A. M., K. Khirallah, H. H. Tawfik, A. Emira, A. K. Abdel Aziz, and S. M. Sedky (2011), Nonlinear dynamics of spring softening and hardening in foldedmems comb drive resonators, Microelectromechanical Systems, Journal of, 20(4), 943-958.

Elvin, N. G., A. A. Elvin, and M. Spector (2001), A self-powered mechanical strain energy sensor, Smart Materials and Structures, 10(2), 293.

Emam, S. A. (2002), A theoretical and experimental study of nonlinear dynamics of buckled beams, Ph.D. thesis, Virginia Polytechnic Institute and State University.

Emam, S. A., and A. H. Nayfeh (2004), On the nonlinear dynamics of a buckled beam subjected to a primary-resonance excitation, Nonlinear Dynamics, 35(1), 1-17.

Ferreira, J. V., and A. L. Serpa (2005), Application of the arc-length method in nonlinear frequency response, Journal of sound and vibration, 284 (1), 133-149.

Fey, R. H. B. (1992), Steady-state behaviour of reduced dynamic systems with local nonlinearities, Ph.D. thesis, Technische Universiteit Eindhoven.

Formica, G., A. Arena, W. Lacarbonara, and H. Dankowicz (2013), Coupling fem with parameter continuation for analysis of bifurcations of periodic responses in nonlinear structures, Journal of Computational and Nonlinear Dynamics, 8(2), 021,013 .

Garmón, F. A., W. T. Ang, P. Khosla, and C. N. Riviere (2003), Rate-dependent inverse hysteresis feedforward controller for microsurgical tool, in Engineering in Medicine and Biology Society, 2003. Proceedings of the 25th Annual International Conference of the IEEE, vol. 4, pp. 3415-3418, IEEE. 
Ge, P., and M. Jouaneh (1995), Modeling hysteresis in piezoceramic actuators, Precision engineering, $17(3), 211-221$.

Genesio, R., and A. Tesi (1992), Harmonic balance methods for the analysis of chaotic dynamics in nonlinear systems, Automatica, 28(3), 531-548.

Ghanea-Hercock, R. (2013), Applied evolutionary algorithms in Java, Springer Science \& Business Media.

Glendinning, P. (1994), Stability, instability and chaos: an introduction to the theory of nonlinear differential equations, Cambridge University Press.

Han, Z.-H., and K.-S. Zhang (2012), Surrogate-Based Optimization, INTECH Open Access Publisher.

Ikeda, T. (1996), Fundamentals of piezoelectricity, Oxford university press.

Jalili, N. (2009), Piezoelectric-based vibration control: from macro to micro/nano scale systems, Springer Science \& Business Media.

Ji, J.-C., and C. Hansen (2000), Non-linear response of a post-buckled beam subjected to a harmonic axial excitation, Journal of Sound and Vibration, 237(2), 303-318.

Jouaneh, M., and H. Tian (1992), Accuracy enhancement of a piezoelectric actuator with hysteresis, in japan/USA symposium on Flexible Automation, vol. 1.

Kenmochi, N., and A. Visintin (1994), Asymptotic stability for nonlinear pdes with hysteresis, European Journal of Applied Mathematics, 5(01), 39-56.

Kerschen, G., J.-c. Golinval, A. F. Vakakis, and L. A. Bergman (2005), The method of proper orthogonal decomposition for dynamical characterization and order reduction of mechanical systems: an overview, Nonlinear dynamics, 41(1-3), 147-169.

Kreider, W., and A. H. Nayfeh (1998), Experimental investigation of single-mode responses in a fixed-fixed buckled beam, Nonlinear Dynamics, 15(2), 155-177.

Kumar, V., J. W. Boley, Y. Yang, H. Ekowaluyo, J. K. Miller, G. T.-C. Chiu, and J. F. Rhoads (2011), Bifurcation-based mass sensing using piezoelectrically-actuated microcantilevers, Applied Physics Letters, 98(15), 153,510.

Kurdi, M., P. Beran, B. Stanford, and R. Snyder (2010), Optimal actuation of nonlinear resonant systems, Structural and Multidisciplinary Optimization, 41(1), 65-86.

Lacarbonara, W. (1997), A theoretical and experimental investigation of nonlinear vibrations of buckled beams, Ph.D. thesis, Virginia Polytechnic Institute and State University.

Lacarbonara, W., and F. Vestroni (2003), Nonclassical responses of oscillators with hysteresis, Nonlinear Dynamics, 32(3), 235-258. 
Leine, R., and N. van de Wouw (2001), Numerical methods for computing periodic solutions, Lecture notes, Department of mechanical engineering, Eindhoven University of Technology.

Lenci, S., and G. Rega (2011), Forced harmonic vibration in a duffing oscillator with negative linear stiffness and linear viscous damping, The Duffing Equation: Nonlinear Oscillators and their Behaviour (eds. I. Kovacic and MJ Brennan), Wiley, ISBN, 830182507, 219-276.

Li, H., S. Preidikman, B. Balachandran, and C. Mote Jr (2006), Nonlinear free and forced oscillations of piezoelectric microresonators, Journal of Micromechanics and Microengineering, 16(2), 356.

Li, S., and R. Batra (2007), Thermal buckling and postbuckling of euler-bernoulli beams supported on nonlinear elastic foundations, AIAA journal, 45(3), 712-720.

Liao, H. (2013), Constrained optimization shooting method for predicting the periodic solutions of nonlinear system, Journal of Computational and Nonlinear Dynamics, $8(4), 041,006$.

Low, T., and W. Guo (1995), Modeling of a three-layer piezoelectric bimorph beam with hysteresis, Microelectromechanical systems, journal of, 4(4), 230-237.

Lynch, S. (2004), Dynamical systems with applications using MATLAB, Springer.

Mack, Y., T. Goel, W. Shyy, and R. Haftka (2007), Surrogate model-based optimization framework: a case study in aerospace design, in Evolutionary computation in dynamic and uncertain environments, pp. 323-342, Springer.

Mallon, N. (2008), Dynamic stability of thin-walled structures: a semi-analytical and experimental approach, Ph.D. thesis, Technische Universiteit Eindhoven.

Melanie, M. (1999), An introduction to genetic algorithms, Cambridge, Massachusetts London, England, Fifth printing, 3.

Mestrom, R. (2009), Multiphysics modelling and experimental validation of microelectromechanical resonator dynamics, Ph.D. thesis, Technische Universiteit Eindhoven.

Mestrom, R. M., R. H. Fey, and H. Nijmeijer (2008), Theoretical and experimental nonlinear dynamics of a clamped-clamped beam mems resonator, Proc. ENOC.

Moheimani, S. R., and A. J. Fleming (2006), Piezoelectric transducers for vibration control and damping, Springer Science \& Business Media.

Mosa, G. A., A. El-naggar, and A. Elbassiouny (2013), Harmonic and sub-harmonic resonance of mems subjected to a weakly non-linear parametric and external excitations, International Journal of Applied Mathematical Research, 2(2), 252-263. 
Nayfeh, A. H., and S. A. Emam (2008), Exact solution and stability of postbuckling configurations of beams, Nonlinear Dynamics, 54(4), 395-408.

Nayfeh, A. H., W. Kreider, and T. Anderson (1995), Investigation of natural frequencies and mode shapes of buckled beams, AIAA journal, 33(6), 1121-1126.

Nguyen, H. X., C. Edeler, and S. Fatikow (2013), Modeling of piezo-actuated stickslip micro-drives: An overview, in Advances in Science and Technology, vol. 81, pp. 39-48, Trans Tech Publ.

Nishawala, V. (2011), A study of large deflection of beams and plates, Ph.D. thesis, Rutgers University-Graduate School-New Brunswick.

Ottman, G. K., H. F. Hofmann, A. C. Bhatt, and G. A. Lesieutre (2002), Adaptive piezoelectric energy harvesting circuit for wireless remote power supply, Power Electronics, IEEE Transactions on, 17(5), 669-676.

Peeters, M., R. Viguié, G. Sérandour, G. Kerschen, and J.-C. Golinval (2008), Nonlinear normal modes, part ii: Practical computation using numerical continuation techniques, in 26th International Modal Analysis Conference.

Pi, Y. L., and N. Trahair (1992), Prebuckling deflections and lateral buckling. ii: applications, Journal of Structural Engineering, 118(11), 2967-2985.

Poon, W.-y. S. (2004), Effect of anti-symmetric mode on dynamic snap-through of curved beam, Ph.D. thesis, The Hong Kong Polytechnic University.

Preisach, F. (1935), Über die magnetische nachwirkung, Zeitschrift für physik, 94(56), 277-302.

Rao, S. S. (2007), Vibration of continuous systems, John Wiley \& Sons.

Rathi, V., and A. H. Khan (2012), Vibration attenuation and shape control of surface mounted, embedded smart beam, Latin American Journal of Solids and Structures, $9(3), 1-25$.

Spottswood, S., and R. Allemang (2007), On the investigation of some parameter identification and experimental modal filtering issues for nonlinear reduced order models, Experimental mechanics, 47(4), 511-521.

Sracic, M. W., and M. S. Allen (2011), Numerical continuation of periodic orbits for harmonically forced nonlinear systems, in Civil Engineering Topics, Volume 4, pp. 51-69, Springer.

Sracic, M. W., S. Yang, and M. S. Allen (2012), Comparing measured and computed nonlinear frequency responses to calibrate nonlinear system models, in Topics in Nonlinear Dynamics, Volume 3, pp. 255-267, Springer. 
Sze, K., S. Chen, and J. Huang (2005), The incremental harmonic balance method for nonlinear vibration of axially moving beams, Journal of Sound and Vibration, $281(3), 611-626$.

Timoshenko, S. P. (1921), Lxvi. on the correction for shear of the differential equation for transverse vibrations of prismatic bars, The London, Edinburgh, and Dublin Philosophical Magazine and Journal of Science, 41(245), 744-746.

Tseng, W.-Y., and J. Dugundji (1970), Nonlinear vibrations of a beam under harmonic excitation, Journal of Applied Mechanics, 37(2), 292-297.

Ugray, Z., L. Lasdon, J. Plummer, F. Glover, J. Kelly, and R. Martí (2007), Scatter search and local nlp solvers: A multistart framework for global optimization, INFORMS Journal on Computing, 19(3), 328-340.

Virgin, L., and R. Plaut (2003), Axial load effects on the frequency response of a clamped beam, in IMAC-XXI: Conference and Exposition on Structural Dynamics.

Virgin, L. N. (2007), Vibration of axially-loaded structures, Cambridge University Press.

Visintin, A. (1994), Difierential models of hysteresis, Applied Mathematical Sciences, 111.

Wagg, D., and S. Neild (2014), Nonlinear vibration with control, Springer.

Waugh, W. H., B. Gallacher, and J. Burdess (2011), A high-sensitivity resonant sensor realized through the exploitation of nonlinear dynamic behaviour, Measurement Science and Technology, 22(10), 105,202.

Weeger, O., U. Wever, and B. Simeon (2013), Isogeometric analysis of nonlinear euler-bernoulli beam vibrations, Nonlinear Dynamics, 72(4), 813-835.

Wolf, K., and O. Gottlieb (2001), Nonlinear dynamics of a cantilever beam actuated by piezoelectric layers in symmetric and asymmetric configuration, Materials Mechanics Laboratory Faculty of Mechanical Engineering, TechnionIIT.

Yabuno, H., M. Ohkuma, and W. Lacarbonara (2003), An experimental investigation of the parametric resonance in a buckled beam, in ASME 2003 International Design Engineering Technical Conferences and Computers and Information in Engineering Conference, pp. 2565-2574, American Society of Mechanical Engineers.

Yamaki, N. a., K. Otomo, and A. Mori (1980), Non-linear vibrations of a clamped beam with initial deflection and initial axial displacement, part ii: Experiment, Journal of Sound and Vibration, 71(3), 347-360.

Younis, M. I. (2011), MEMS Linear and Nonlinear Statics and Dynamics: Mems Linear and Nonlinear Statics and Dynamics, vol. 20, Springer Science \& Business Media. 